Supporting Information

\title{
Solvent-free mechanochemical diaza-Cope rearrangement
}

Wenxian Ma, ${ }^{\text {abc }}$ Yan Liu, ${ }^{\text {a }} \mathrm{Na}$ Yu, ${ }^{\text {a KaKing } \text { Yan }}{ }^{* a}$

a. School of Physical Science and Technology, ShanghaiTech University, Shanghai 201210, China.

b. Shanghai Advanced Research Institute, Chinese Academy of Sciences, Shanghai 201203, China.

c. University of Chinese Academy of Sciences, Beijing 100049, China.

\section{Table of Ccontents}

1. General information

2. Optimization of different ball-milling conditions of diaza-Cope rearrangement (Table S1S6)

3. General procedure for mechanochemical diaza-Cope rearrangement followed by acid hydrolysis to chiral diammonium salts (Figure S2-S62)

4. Substrates tested that are not suitable for mechanochemical diaza-Cope rearrangement (Figure S63)

5. General procedure of solvent-free mechanochemical one-pot two-step chiral $\mathrm{Zn}$ salen complexes (Figure S64-S67)

6. Kinetic profile of different reaction techniques with $\mathbf{1}$ and $\mathbf{2 a}$ (Figure S68-S75)

7. Comparison of benzocrown substrate in diaza-Cope rearrangement: ball-milling vs. solution (Figure S76)

8. PXRD studies (Figure S77-S79)

9. HPLC of aging experiments of diaza-Cope rearrangement of $\mathbf{1}$ and 2a (Figure S80)

10. Single-crystal X-ray diffraction analysis of 3I (Figure S81)

11. Temperature monitoring during mechanochemical conditions (Table S7-S10)

12. Reference 


\section{General information}

\section{Reagents and equipment}

All commercial reagents and solvents were used as received unless otherwise indicated. $(1 R, 2 R)$ 1,2-Bis(2-hydroxyphenyl)ethylenediamine (95\%) and (1S,2S)-1,2-bis(2-

hydroxyphenyl)ethylenediamine (95\%) were purchased from Sigma Aldrich. Other reagents and solvents were purchased from Sinopharm Chemical Reagent, Bidepharm, TCI, Energy Chemical and J\&K Chemical. Deuterated solvents were acquired from Cambridge Isotope Laboratories, Inc, Adamas, and Eurisotop. Chromatographic purification of products was accomplished by flash chromatography using silica gel. Thin-layer chromatography (TLC) was performed on ShanXi NuoTai silica gel SHF254 TLC plates.

NMR spectra were obtained from Bruker Avance NEO 400 and Bruker Avance III HD 500. ${ }^{1} \mathrm{H}$ NMR spectra were recorded at $500 \mathrm{MHz}$ or $400 \mathrm{MHz},{ }^{19} \mathrm{~F}$ NMR were recorded at $471 \mathrm{MHz}$, and ${ }^{13} \mathrm{C}$ NMR spectra were recorded at $125 \mathrm{MHz}$ or $100 \mathrm{MHz} .{ }^{1} \mathrm{H}$ NMR chemical shifts were determined relative to the signal of the residual protonated solvent. Data for ${ }^{1} \mathrm{H}$ NMR are reported as follows: chemical shift $(\delta$ ppm $)$, multiplicity $(\mathrm{s}=$ singlet, $\mathrm{d}=$ doublet, $\mathrm{t}=$ triplet, $\mathrm{m}=$ multiplet). ${ }^{13} \mathrm{C}\left\{{ }^{1} \mathrm{H}\right\}$ NMR chemical shifts NMR spectra were reported as chemical shifts in ppm and multiplicity where appropriate.

High Resolution Mass spectra were obtained from Thermo Fisher Q-Exactive high-resolution MS, operated in ESI mode. Enantiomeric excesses were determined on an Agilent 1260 Chiral HPLC using IC, and OD columns. Powder X-ray diffraction (PXRD) patterns were collected on a X-ray diffractometers (Bruker D2 PHASER or D8 ADVANCE).

Crystals were coated with Paratone-N oil and mounted on a Bruker D8 Venture diffractometer with multilayer optic-monochromatic MetalJet $\mathrm{Ga}-\mathrm{K} \alpha$ radiation $(\lambda=1.34138 \AA)$. The crystal was kept at $150 \mathrm{~K}$ during data collection. Using Olex $2,{ }^{2}$ the structure was solved with the ShelXT ${ }^{3}$ structure solution program using Intrinsic Phasing and refined with the $\mathrm{XL}^{4}$ refinement package using Least Squares minimization. 
Ball-milling reactions were performed with a Shanghai Jing Xin JXFSTPRP-24 mixer mill. Polypropylene (PP) milling tubes $(2 \mathrm{ml}, 5 \mathrm{ml})$, stainless steel milling vessels ( $2 \mathrm{ml}$ (JX-

KG0177), $5 \mathrm{ml}$ (JX-KG0178), $15 \mathrm{ml}$ (JX-KG0180)), and grinding balls (3.2 mm (JX-GZ0137), $5.2 \mathrm{~mm}$ (JX-GZ0138), $8.0 \mathrm{~mm}$ ((JX-GZ0140), $12.0 \mathrm{~mm}$ (JX-GZ0142)) were purchased from Shanghai Jing Xin.

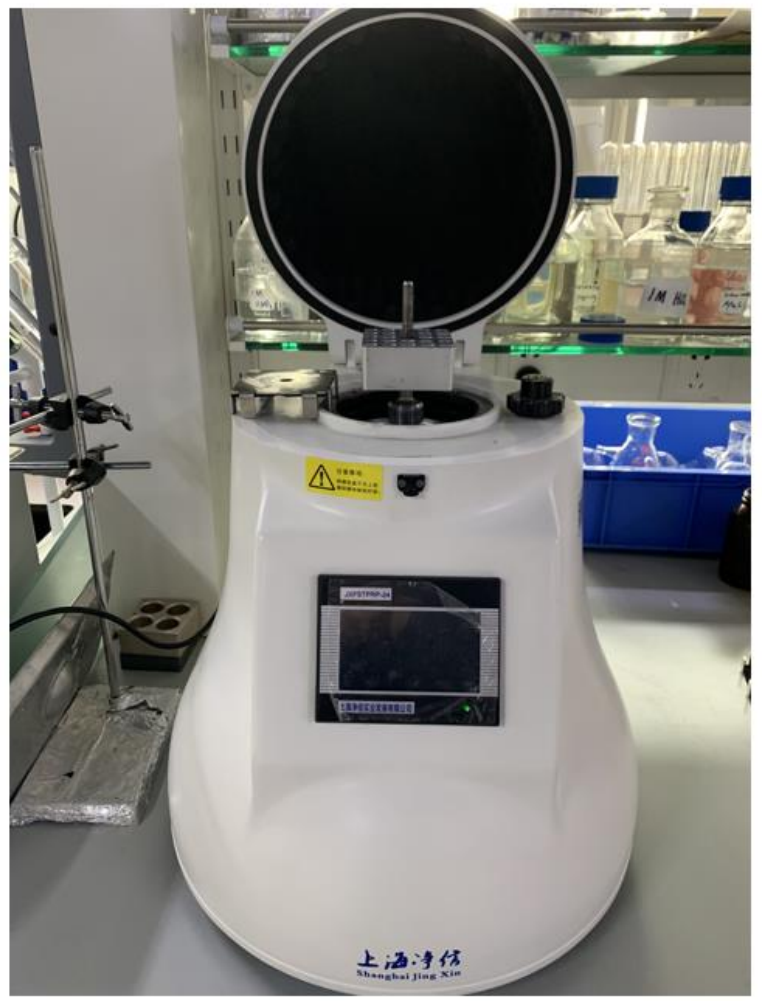

\section{Shanghai Jing Xin JXFSTPRP-24 mixer mill}
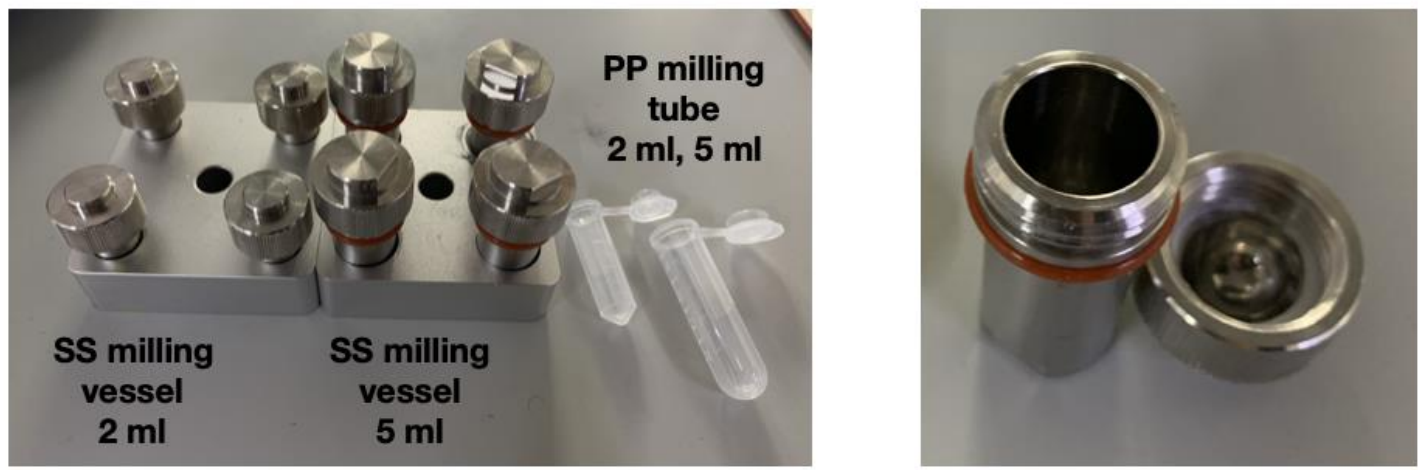

Fig S1. (Top) Ball-milling machine, and (bottom) ball-milling vessels (both stainless steel and PP) used in this study. 
2. Optimization of different ball-milling conditions of diaza-Cope rearrangement

a) Table S1. Grinding container material, grinding ball material

\begin{tabular}{|c|ccccc|}
\hline Size of grinding \\
ball (mm)
\end{tabular}

${ }^{a}$ Container material: $\mathrm{PP}=$ polypropylene centrifuge tube; $\mathrm{SS}=$ stainless steel grinding container. ${ }^{b} \mathrm{SS}=$ stainless steel grinding ball. ${ }^{c}{ }^{1} \mathrm{H}$ NMR spectrum is taken immediately after ball-milling reaction at the reported time. Yield is determined by comparing the ${ }^{1} \mathrm{H}$ NMR integration of the diaza-Cope product with that of an internal standard (dibromomethane) with known concentration.

Comment: The combination of stainless steel container and grinding balls tend to provide a better reaction conversion. 
b) Table S2. Grinding container volume

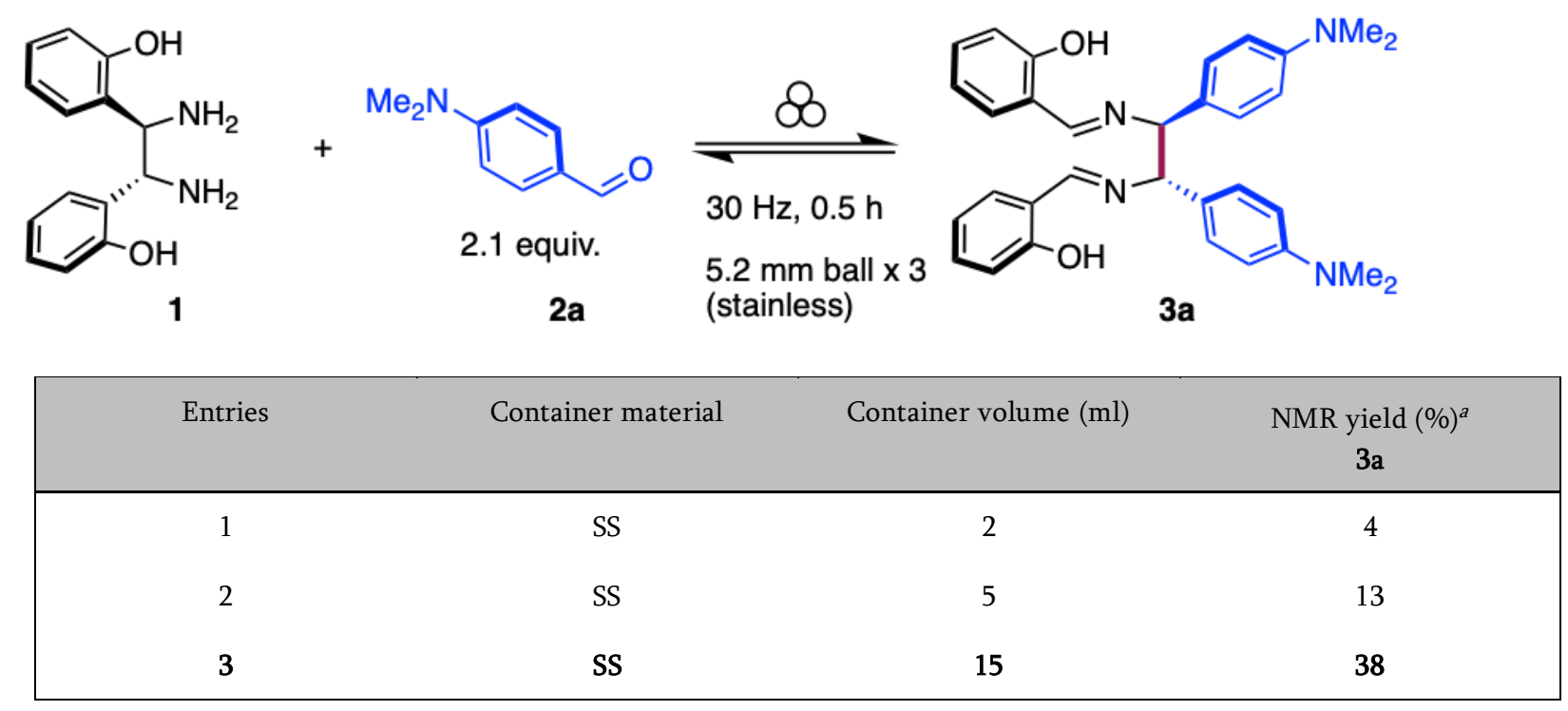

${ }^{\text {a }}$ Yield is determined by comparing the ${ }^{1} \mathrm{H}$ NMR integration of the diaza-Cope product with that of an internal standard (dibromomethane) with known concentration.

Comment: Grinding container of bigger volume gives better reaction conversion. 
c) Table S3. Grinding balls size

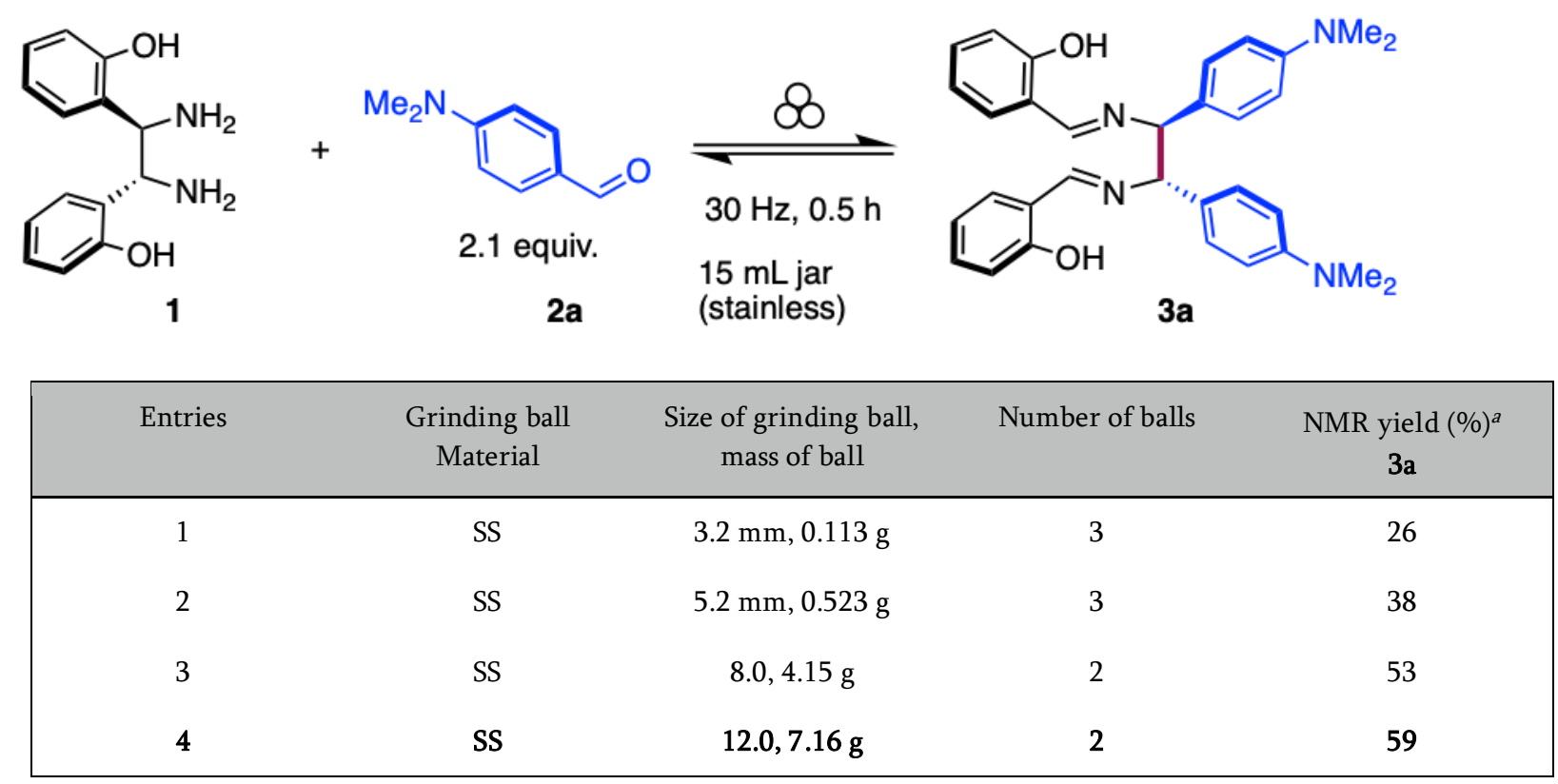

${ }^{a}$ Yield is determined by comparing the ${ }^{1} \mathrm{H}$ NMR integration of the diaza-Cope product with that of an internal standard (dibromomethane) with known concentration.

Comment: Grinding container of bigger volume gives better reaction conversion. 
d) Table S4. Grinding time

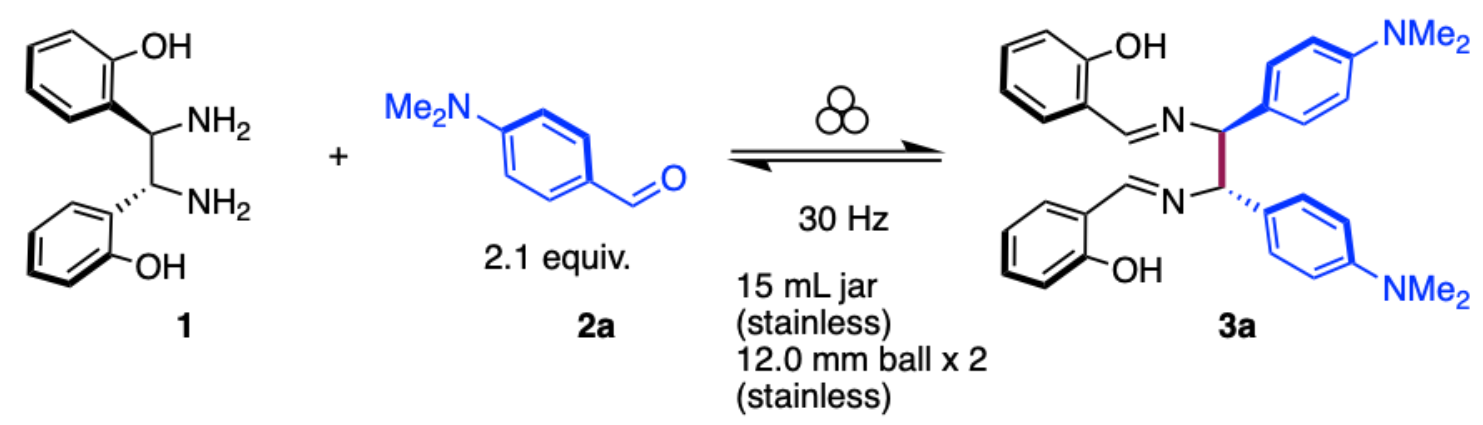

\begin{tabular}{|ccc|}
\hline Entries & $\begin{array}{c}\text { Time } \\
(\mathrm{h})\end{array}$ & $\begin{array}{c}\text { NMR yield }(\%)^{a} \\
\text { 3a }\end{array}$ \\
\hline 1 & 0.5 & 59 \\
2 & 1 & 69 \\
3 & 2 & 76 \\
4 & 3 & 82 \\
$\mathbf{5}$ & 4 & 96 \\
\hline
\end{tabular}

${ }^{a}$ Yield is determined by comparing the ${ }^{1} \mathrm{H}$ NMR integration of the diaza-Cope product with that of an internal standard (dibromomethane) with known concentration.

Comment: The longer the ball-milling time gives better reaction conversion. 
e) Table S5. Ball-milling frequency

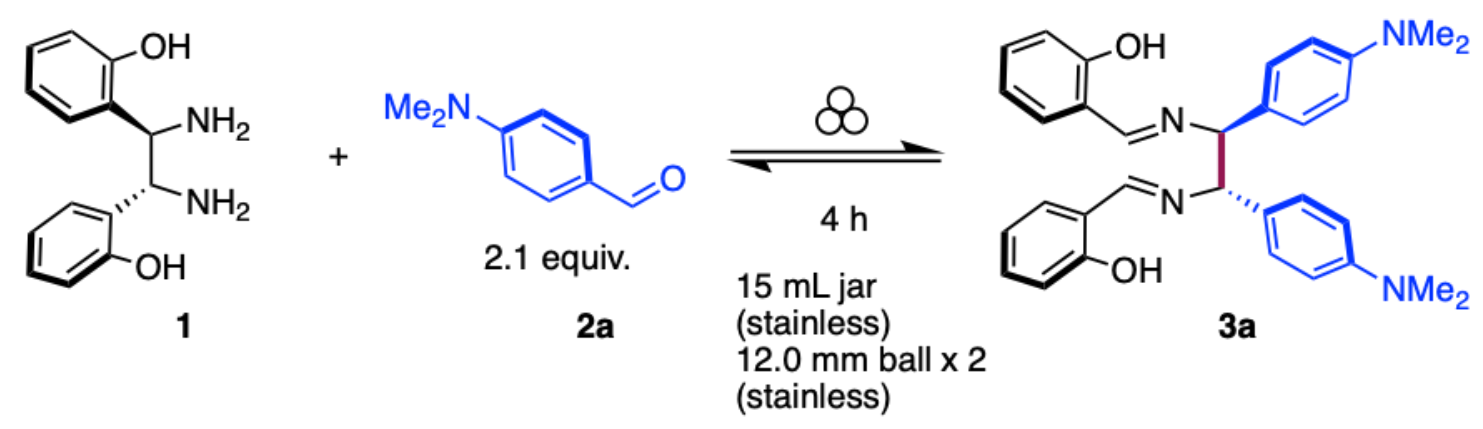

\begin{tabular}{|ccc|}
\hline Entries & $\begin{array}{c}\text { Frequency } \\
(\mathrm{Hz})\end{array}$ & $\begin{array}{c}\text { NMR yield }(\%)^{a} \\
\text { 3a }\end{array}$ \\
\hline 1 & 5 & 42 \\
2 & 10 & 66 \\
3 & 20 & 74 \\
4 & 30 & 96 \\
5 & 40 & 95 \\
6 & 50 & 95 \\
\hline
\end{tabular}

${ }^{a}$ Yield is determined by comparing the ${ }^{1} \mathrm{H}$ NMR integration of the diaza-Cope product with that of an internal standard (dibromomethane) with known concentration.

Comment: Higher ball-milling frequency gives better reaction conversion, but frequency of 30$50 \mathrm{~Hz}$ gives essentially the same reaction conversion for $4 \mathrm{~h}$ of ball-milling time. 
f) Table S6. Number of grinding ball<smiles>NC(c1ccccc1O)[C@H](N)c1ccccc1O</smiles>

1<smiles>CN(C)c1ccc(C=O)cc1</smiles>

2.1 equiv.

2a

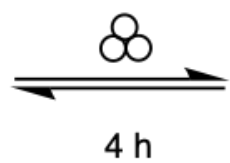

$15 \mathrm{~mL}$ jar (stainless) $12.0 \mathrm{~mm}$ ball (stainless)<smiles>Cc1ccc([C@@H](/N=C/c2ccccc2O)C(/N=C/c2ccccc2O)c2ccc(N(C)C)cc2)cc1</smiles>

3a

\begin{tabular}{|ccc|}
\hline Entries & Number of balls & $\begin{array}{c}\text { NMR yield (\%) } \\
\text { 3a }\end{array}$ \\
\hline 1 & 1 & 96 \\
2 & 2 & 95 \\
3 & 3 & 96 \\
\hline
\end{tabular}

${ }^{a}$ Yield is determined by comparing the ${ }^{1} \mathrm{H}$ NMR integration of the diaza-Cope product with that of an internal standard (dibromomethane) with known concentration.

Comment: The reaction conversion is not directly influenced by the number of stainless-steel grinding balls. 
3. General procedure for mechanochemical diaza-Cope rearrangement

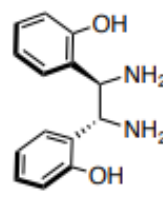

(R, R) 1

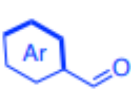

2

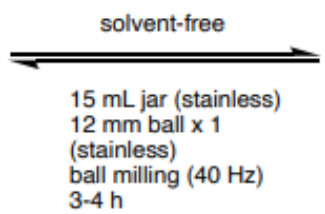

ball milling $(40 \mathrm{~Hz})$

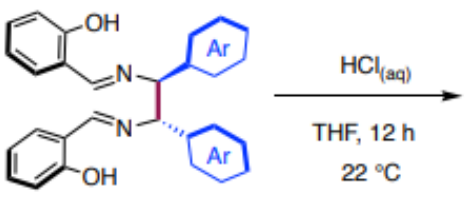

$(S, S) 3$

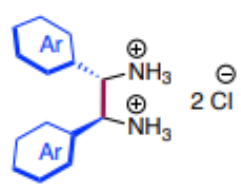

$(S, S) 4$

(1R,2R)-1,2-Bis(2-hydroxyphenyl)ethylenediamine 1, arylaldehyde $\mathbf{2}$ (2.1 equiv.) were placed in a stainless steel ball-milling vessel $(15 \mathrm{~mL})$ loaded with one grinding ball (stainless steel, diameter: $12 \mathrm{~mm}$ ). After the vessel was closed in open air, the vessel was placed in the ball mill machine. After $4 \mathrm{~h}$ of ball-milling at $40 \mathrm{~Hz}$, with a periodic 5 min pause for every $1 \mathrm{~h}$ of milling, the solid residual was dissolved in THF $(10 \mathrm{~mL})$, followed by slow addition of conc.

hydrochloric acid (12 M, $0.5 \mathrm{~mL})$. White precipitate was formed upon acidic workup, and this heterogeneous mixture was stirred at $\mathrm{rt}$ for overnight. After the reaction, the white precipitate was filtered, and was washed with copious amount of THF $(10 \mathrm{~mL} \times 3)$, ethyl lactate $(10 \mathrm{~mL} \times$ 3) and DCM (10 mL x 3). The residual was dried under reduced pressure to give 4 as an analytically pure solid. 


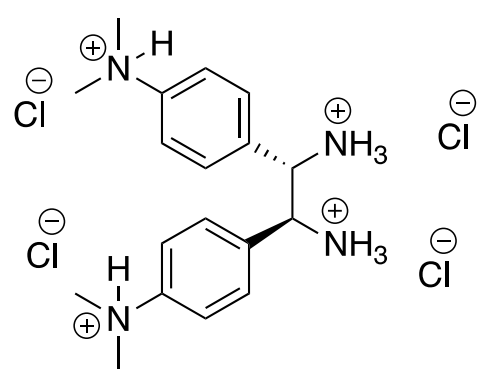

Prepared according to general procedure from 1 (50 mg, $0.20 \mathrm{mmol}$ ) and 4-aminobenzaldehyde (2a) (64 mg, $0.43 \mathrm{mmol}$ ); washed with DCM, EA and THF to give the title compound as a white solid (72 mg, 80\%).

${ }^{1} \mathrm{H}$ NMR $\left(\mathrm{D}_{2} \mathrm{O}, 400 \mathrm{MHz}, 25{ }^{\circ} \mathrm{C}\right): \delta 7.61(\mathrm{~d}, J=8.4 \mathrm{~Hz}, 4 \mathrm{H}), 7.51(\mathrm{~d}, J=8.5 \mathrm{~Hz}, 4 \mathrm{H}), 5.21(\mathrm{~s}, 2$ $\mathrm{H}), 3.24$ (s, $12 \mathrm{H})$.

${ }^{13} \mathrm{C}\left\{{ }^{1} \mathrm{H}\right\}$ NMR $\left(\mathrm{D}_{2} \mathrm{O}, 101 \mathrm{MHz}, 25{ }^{\circ} \mathrm{C}\right) \delta 143.43,133.22,130.47,121.63,56.25,46.17$.

HRMS (ESI) calculated for $\mathrm{C}_{18} \mathrm{H}_{24} \mathrm{~N}_{3},[\mathrm{M}+\mathrm{H}]^{+}: 282.1965$, Found: 282.1956 due to escape of $\mathrm{NH}_{3}$ under mass spec. condition.

HPLC analysis condition:

Solvent: $n$-hexane/ iso-propanol $=95: 5$

Column: CHIRALPAK IC column (Nomura Chemical Co., Lid.; inner diameter $4.6 \mathrm{~mm} \times$ length 250mm; particle size $=5 \mu \mathrm{m}$ )

Column temperature: $25^{\circ} \mathrm{C}$

Detector: UV at $350 \mathrm{~nm}$

Flow rate: $1.0 \mathrm{~mL} / \mathrm{min}$

Injection volume: $5 \mu \mathrm{L}$

$(R, R)-\mathbf{4 a} t_{\mathrm{R}}=10.0 \mathrm{~min},(S, S)-\mathbf{4 a} \mathrm{t}_{\mathrm{R}}=14.0 \mathrm{~min}$ for racemic sample of $\mathbf{4 a}$.

ee $>99 \%$ 


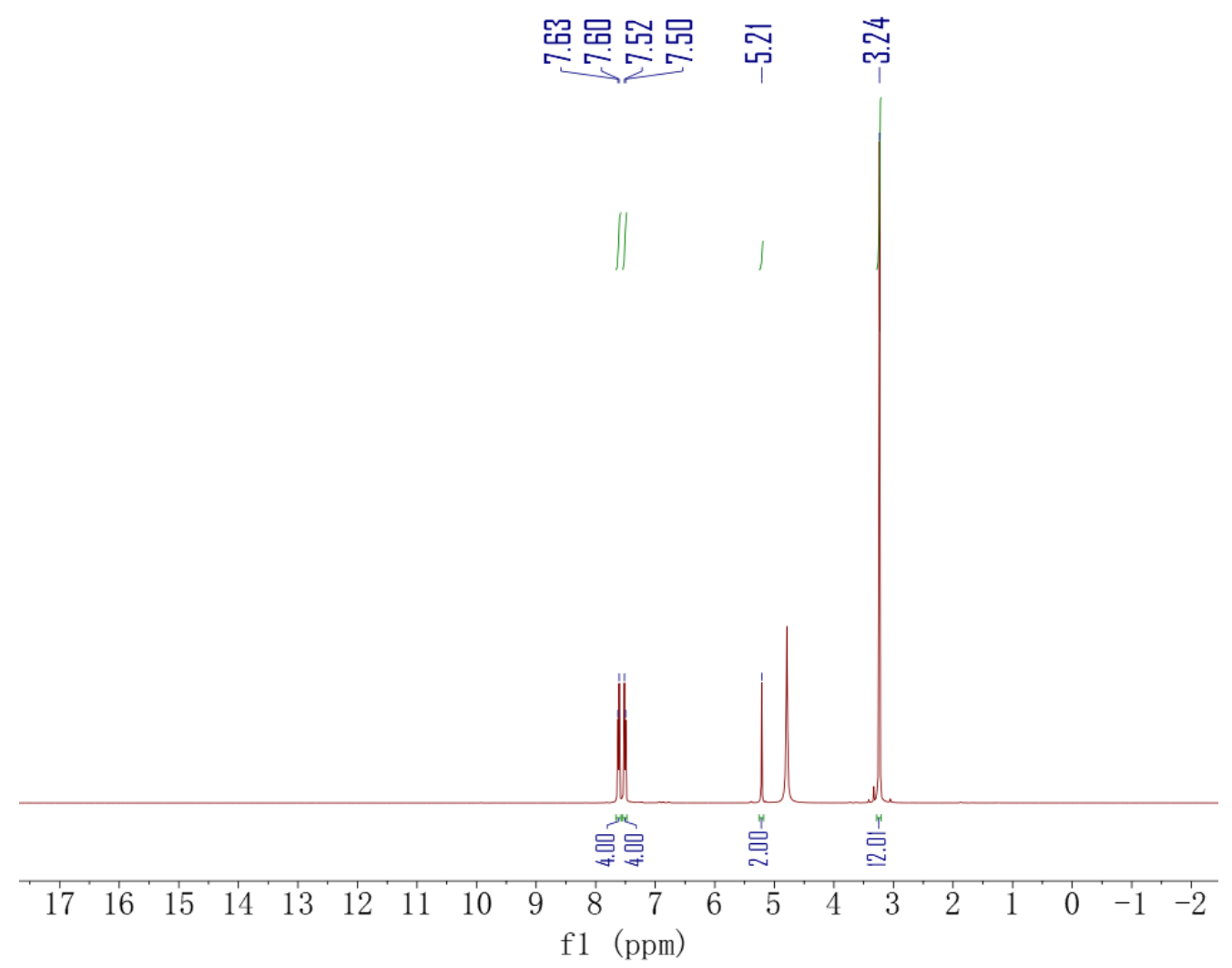

Figure S2. ${ }^{1} \mathrm{H}$ NMR of $\mathbf{4 a}$
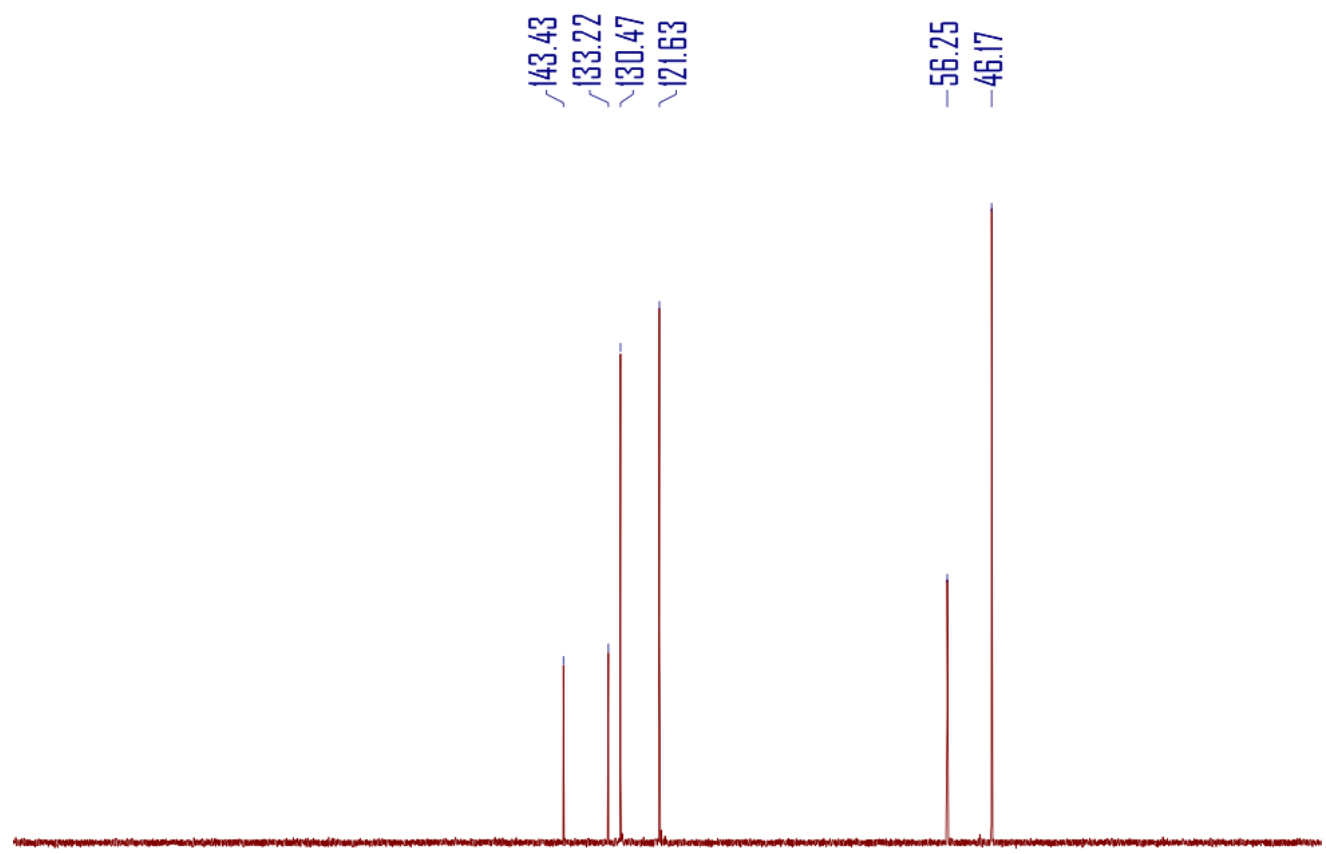

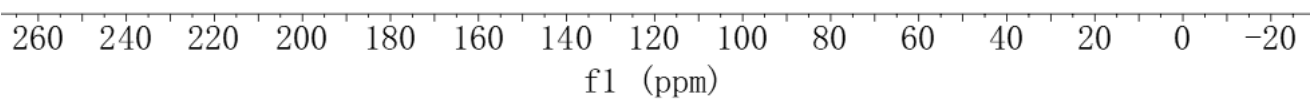

Figure S3. ${ }^{13} \mathrm{C}\left\{{ }^{1} \mathrm{H}\right\}$ NMR of $\mathbf{4 a}$. 

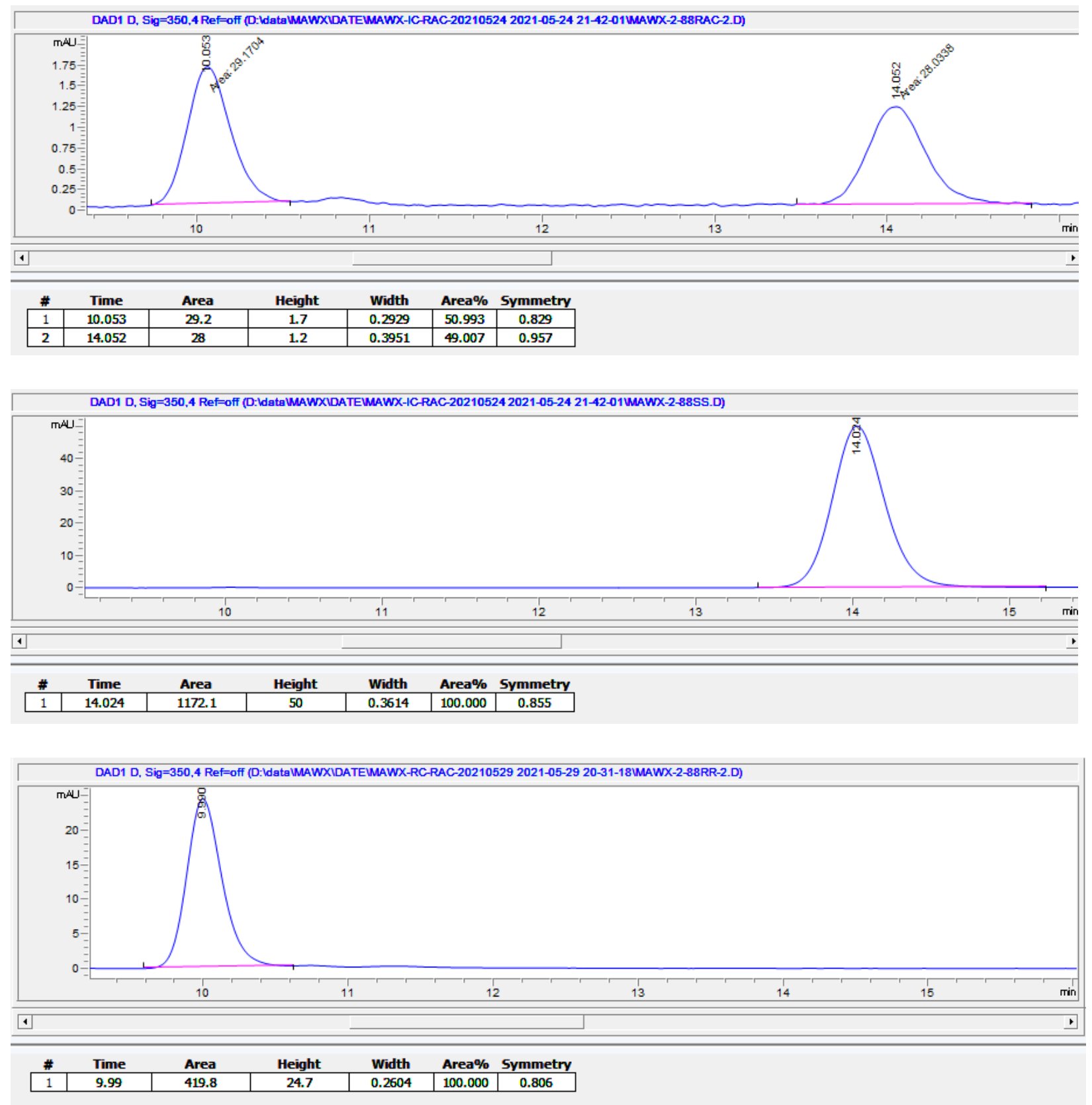

Figure S4. HPLC analysis of (top) racemic sample; (middle) $(S, S)$ sample, and (bottom) $(R, R)$ sample of $\mathbf{4 a}$. 


\section{$(1 S, 2 S)$-1,2-diphenylethylenediamine dihydrochloride (4b)}

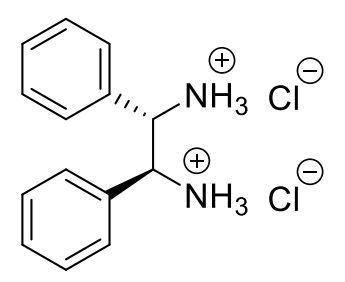

Prepared according to general procedure from $1(50 \mathrm{mg}, 0.20 \mathrm{mmol})$ and benzaldehyde (2b) (46 $\mathrm{mg}, 0.43 \mathrm{mmol}$ ); washed with DCM, EA and THF to give the title compound as a white solid (50 $\mathrm{mg}, 85 \%)$.

${ }^{1} \mathrm{H}$ NMR (DMSO- $\left.d_{6}, 400 \mathrm{MHz}, 25^{\circ} \mathrm{C}\right): \delta 9.40(\mathrm{~s}, 6 \mathrm{H}), 7.41-7.30(\mathrm{~m}, 4 \mathrm{H}), 7.28-7.17(\mathrm{~m}, 6 \mathrm{H})$, $5.11(\mathrm{~s}, 2 \mathrm{H})$.

${ }^{13} \mathrm{C}\left\{{ }^{1} \mathrm{H}\right\}$ NMR (DMSO- $\left.d_{6}, 101 \mathrm{MHz}, 25{ }^{\circ} \mathrm{C}\right) \delta 133.39,128.95,128.75,128.40,56.94$.

HRMS (ESI) calculated for $\mathrm{C}_{14} \mathrm{H}_{17} \mathrm{~N}_{2},[\mathrm{M}+\mathrm{H}]^{+}:$213.1386, Found: 213.1379 .

HPLC analysis condition:

Solvent: n-hexane/ iso-propanol $=99: 1$

Column: CHIRALPAK IC column (Nomura Chemical Co., Lid.; inner diameter $4.6 \mathrm{~mm} \times$ length 250mm; particle size $=5 \mu \mathrm{m}$ )

Column temperature: $25{ }^{\circ} \mathrm{C}$

Detector: UV at $254 \mathrm{~nm}$

Flow rate: $0.5 \mathrm{~mL} / \mathrm{min}$

Injection volume: $5 \mu \mathrm{L}$

$(R, R)-\mathbf{4 b} t_{\mathrm{R}}=12.4 \mathrm{~min},(S, S)-\mathbf{4 b} \mathrm{t}_{\mathrm{R}}=17.1 \mathrm{~min}$ for racemic sample of $\mathbf{4 b}$.

ee $>99 \%$ 


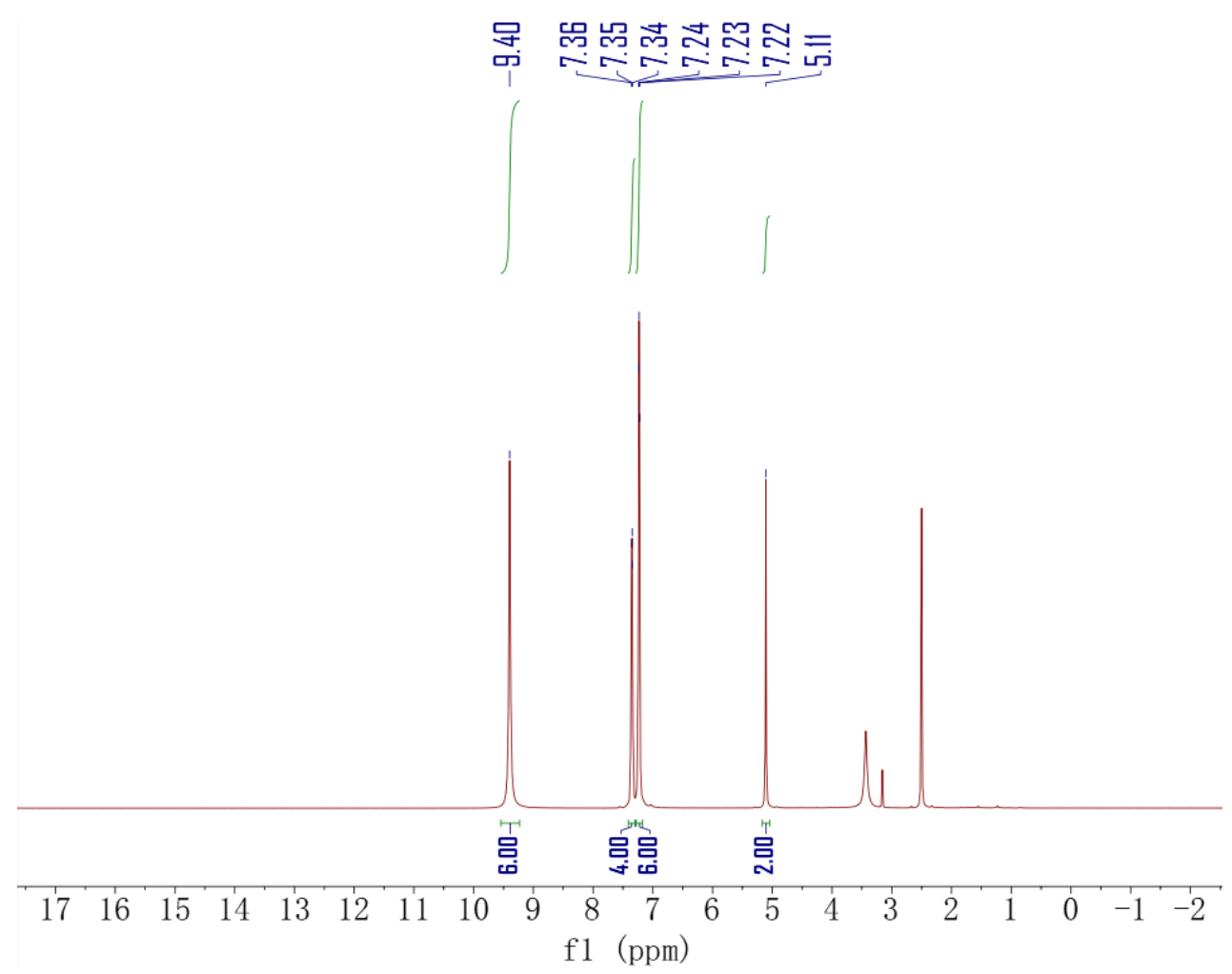

Figure S5. ${ }^{1} \mathrm{H}$ NMR of $\mathbf{4 b}$.

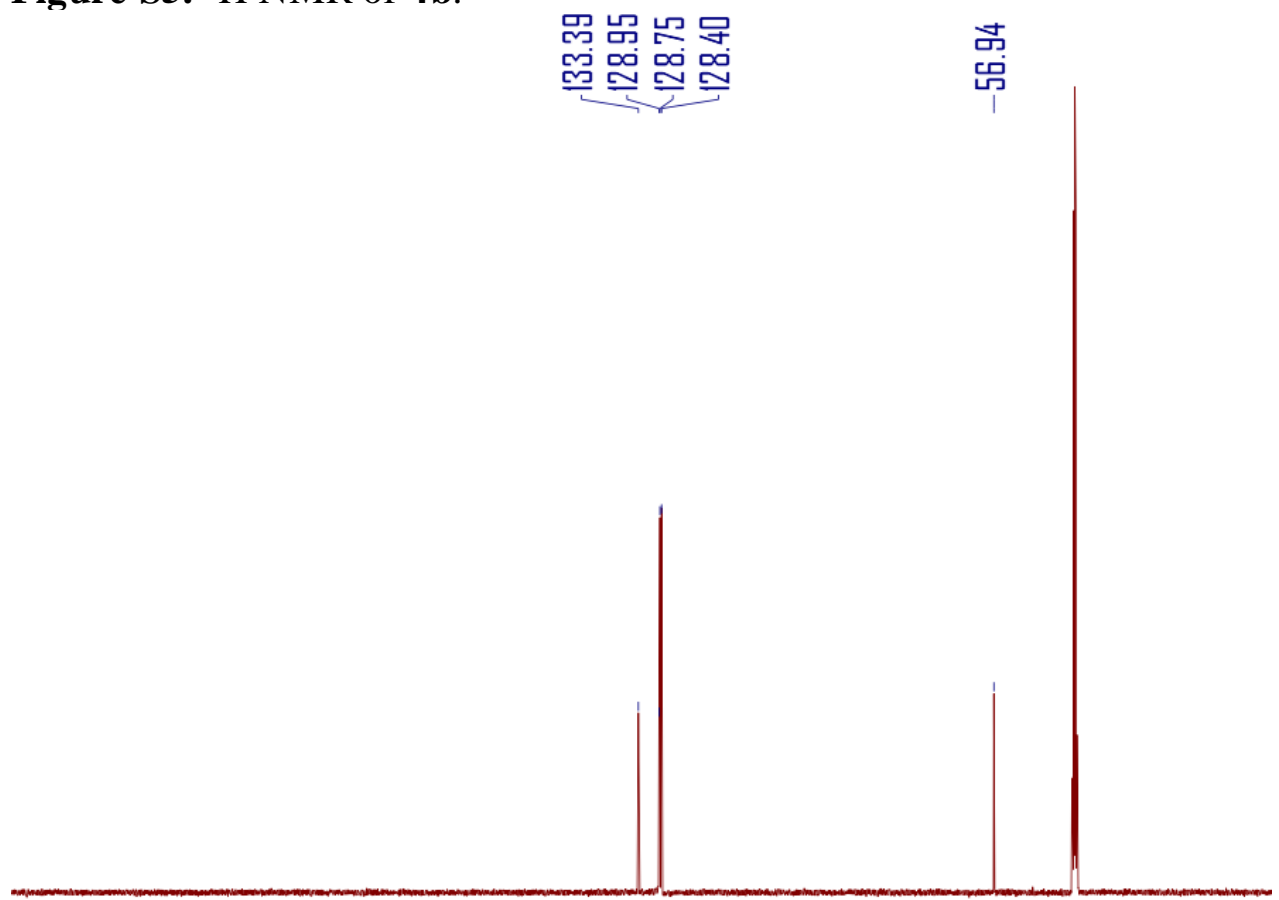

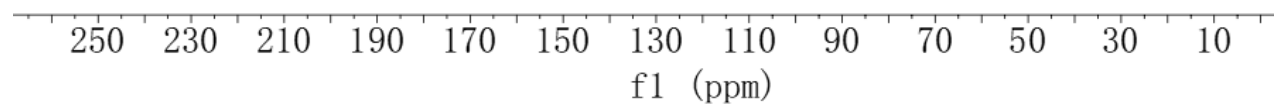

Figure S6. ${ }^{13} \mathrm{C}\left\{{ }^{1} \mathrm{H}\right\}$ NMR of $\mathbf{4 b}$. 

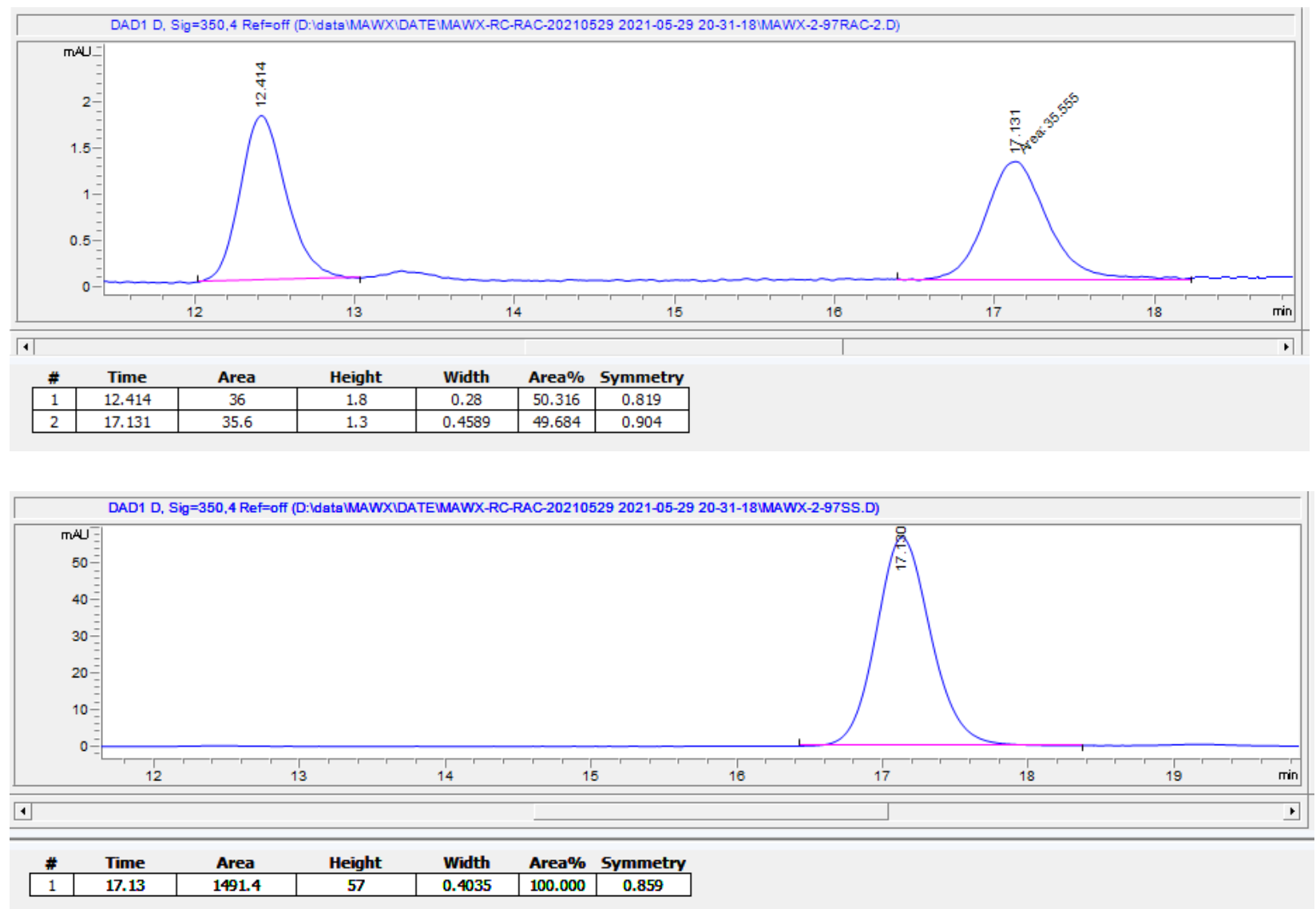

Figure S7. HPLC analysis of (top) racemic sample, and (bottom) $(S, S)$ sample of $\mathbf{4 b}$. 


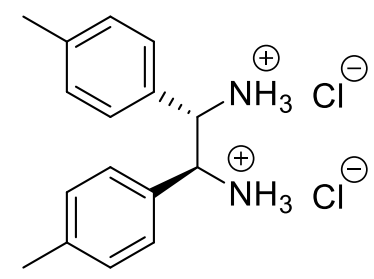

Prepared according to general procedure from $1(50 \mathrm{mg}, 0.20 \mathrm{mmol})$ and 4-methylbenzaldehyde (2c) $(52 \mathrm{mg}, 0.43 \mathrm{mmol})$; washed with DCM, EA and THF to give the title compound as a white solid (57 mg, 89\%).

${ }^{1} \mathrm{H}$ NMR (DMSO- $\left.d_{6}, 500 \mathrm{MHz}, 25^{\circ} \mathrm{C}\right): \delta 9.17$ (s, $\left.6 \mathrm{H}\right), 7.23-7.18(\mathrm{~m}, 4 \mathrm{H}), 7.08(\mathrm{~d}, J=7.9 \mathrm{~Hz}, 4$ $\mathrm{H}), 4.99(\mathrm{~s}, 2 \mathrm{H}), 2.22(\mathrm{~s}, 6 \mathrm{H})$.

${ }^{13} \mathrm{C}\left\{{ }^{1} \mathrm{H}\right\}$ NMR (DMSO- $\left.d_{6}, 101 \mathrm{MHz}, 25{ }^{\circ} \mathrm{C}\right) \delta 138.37,130.31,129.02,128.62,56.49,20.68$.

HRMS (ESI) calculated for $\mathrm{C}_{16} \mathrm{H}_{21} \mathrm{~N}_{2},[\mathrm{M}+\mathrm{H}]^{+}:$241.1699, Found: 241.1690 .

HPLC analysis condition:

Solvent: $n$-hexane/ iso-propanol $=80: 20$

Column: CHIRALPAK IC column (Nomura Chemical Co., Ltd.; inner diameter $4.6 \mathrm{~mm} \times$ length $250 \mathrm{~mm}$; particle size $=5 \mu \mathrm{m}$ )

Column temperature: $25^{\circ} \mathrm{C}$

Detector: UV at $350 \mathrm{~nm}$

Flow rate: $0.8 \mathrm{~mL} / \mathrm{min}$

Injection volume: $5 \mu \mathrm{L}$

$(R, R)-\mathbf{4 c} \mathrm{t}_{\mathrm{R}}=7.2 \mathrm{~min},(S, S)-\mathbf{4 c} \mathrm{t}_{\mathrm{R}}=8.2 \mathrm{~min}$ for racemic sample of $\mathbf{4 c}$.

ee $>99 \%$ 


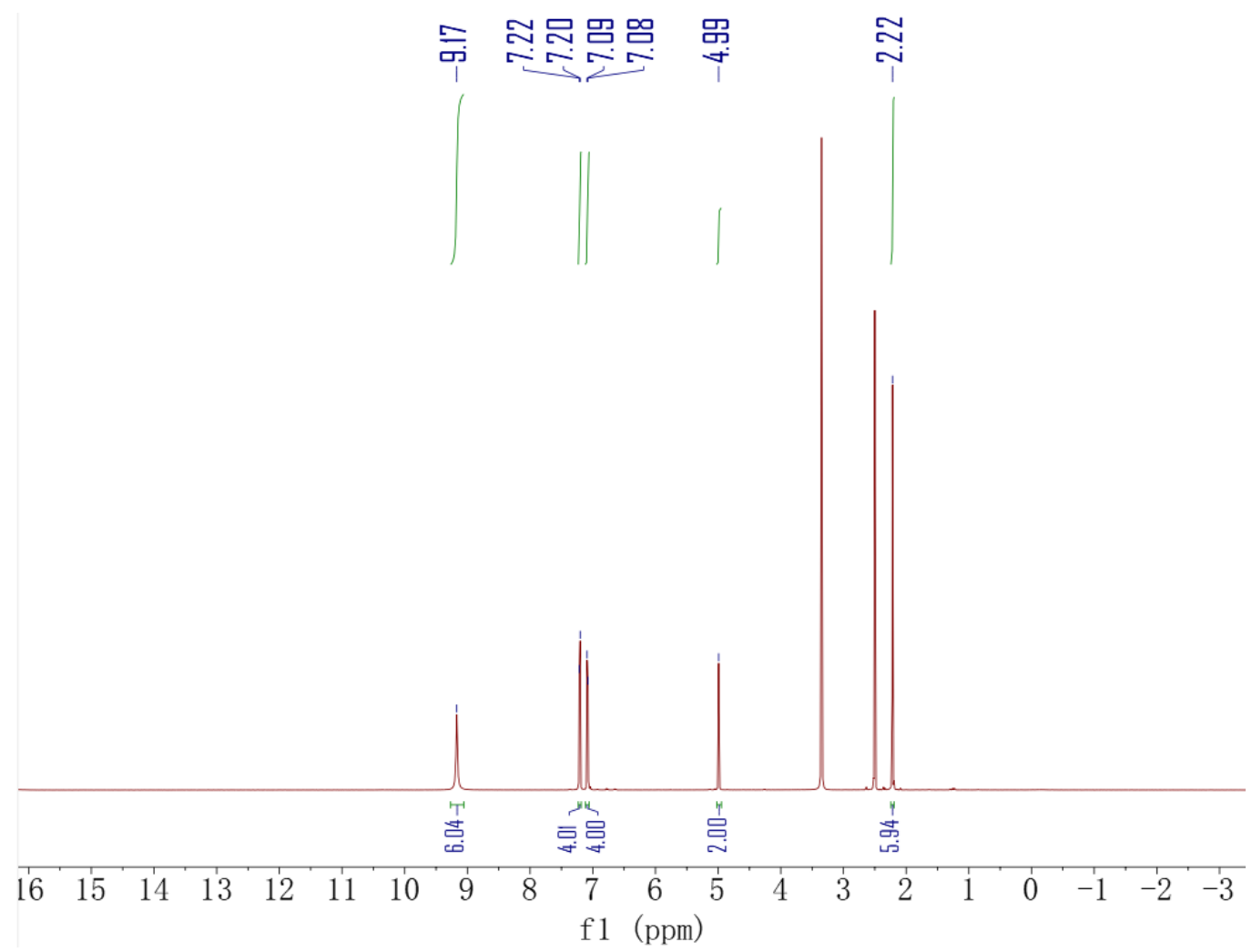

Figure S8. ${ }^{1} \mathrm{H}$ NMR of $\mathbf{4 c}$.

ติ 돔

哭岢器器

吕鬲品

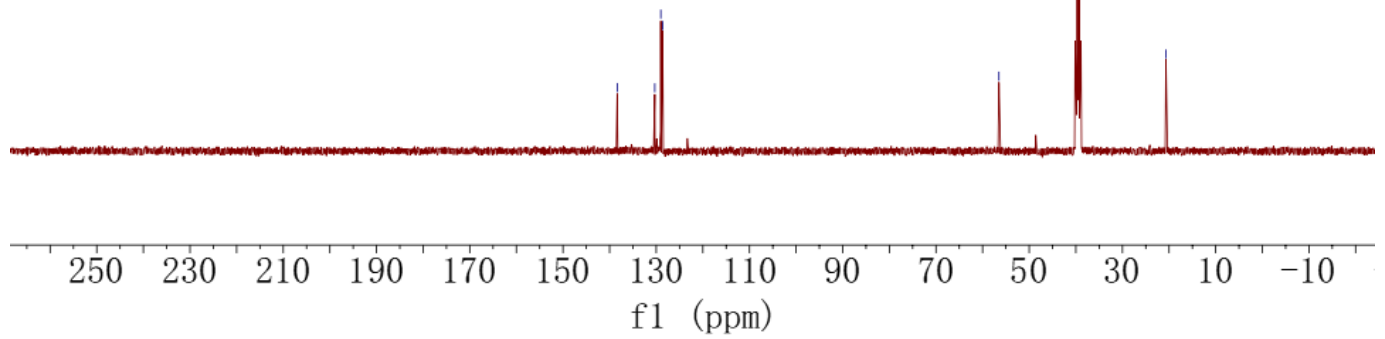

Figure S9. ${ }^{13} \mathrm{C}\left\{{ }^{1} \mathrm{H}\right\}$ NMR of $\mathbf{4 c}$. 

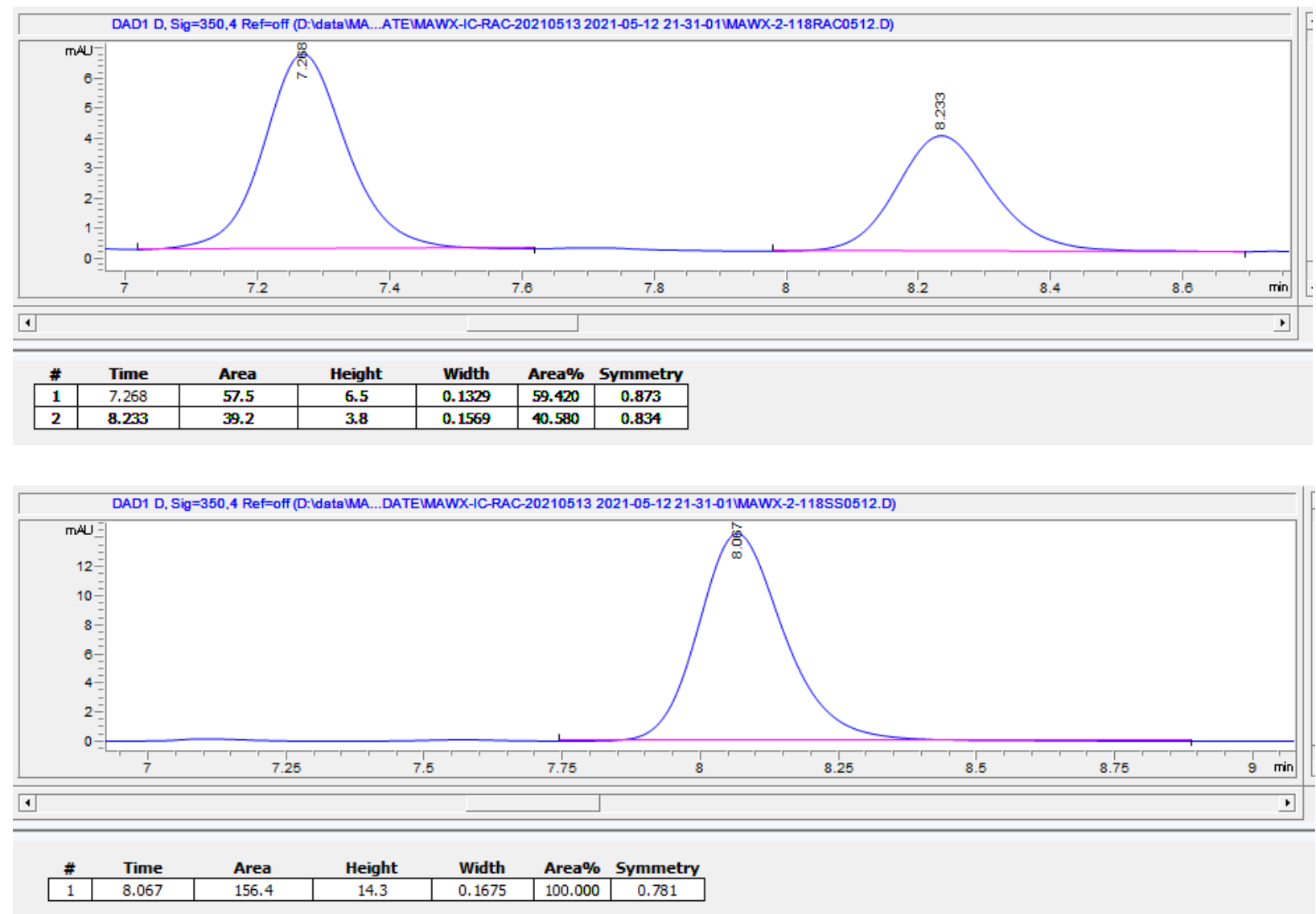

Figure S10. HPLC analysis of (top) racemic sample, and (bottom) $(S, S)$ sample of $4 \mathbf{c}$. 


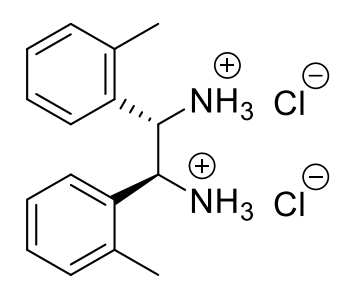

Prepared according to general procedure from $1(50 \mathrm{mg}, 0.20 \mathrm{mmol})$ and 2-methylbenzaldehyde (2d) $(52 \mathrm{mg}, 0.43 \mathrm{mmol})$; washed with DCM, EA and THF to give the title compound as a white solid (55 mg, 86\%).

${ }^{1} \mathrm{H}$ NMR (DMSO- $\left.d_{6}, 400 \mathrm{MHz}, 25{ }^{\circ} \mathrm{C}\right): \delta 9.26(\mathrm{~s}, 6 \mathrm{H}), 7.76(\mathrm{~d}, J=7.8 \mathrm{~Hz}, 2 \mathrm{H}), 7.17(\mathrm{t}, J=7.5$ $\mathrm{Hz}, 2 \mathrm{H}), 7.10(\mathrm{t}, J=7.4 \mathrm{~Hz}, 2 \mathrm{H}), 7.00(\mathrm{~d}, J=7.6 \mathrm{~Hz}, 2 \mathrm{H}), 5.33(\mathrm{~s}, 2 \mathrm{H}), 2.16(\mathrm{~s}, 6 \mathrm{H})$.

${ }^{13} \mathrm{C}\left\{{ }^{1} \mathrm{H}\right\}$ NMR (DMSO- $\left.d_{6}, 101 \mathrm{MHz}, 25{ }^{\circ} \mathrm{C}\right) \delta 136.32,132.83,130.43,128.94,127.16,126.23$, 52.77, 19.19.

HRMS (ESI) calculated for $\mathrm{C}_{16} \mathrm{H}_{21} \mathrm{~N}_{2},[\mathrm{M}+\mathrm{H}]^{+}:$241.1699, Found: 241.1688.

HPLC analysis condition:

Solvent: n-hexane/ iso-propanol $=98: 2$

Column: CHIRALPAK IC column (Nomura Chemical Co., Ltd.; inner diameter $4.6 \mathrm{~mm} \times$ length $250 \mathrm{~mm}$; particle size $=5 \mu \mathrm{m}$ )

Column temperature: $25^{\circ} \mathrm{C}$

Detector: UV at $254 \mathrm{~nm}$

Flow rate: $0.5 \mathrm{~mL} / \mathrm{min}$

Injection volume: $5 \mu \mathrm{L}$

$(S, S)-\mathbf{4} \mathbf{d} t_{\mathrm{R}}=21.2 \mathrm{~min},(R, R)-\mathbf{4} \mathbf{d} \mathrm{t}_{\mathrm{R}}=23.2 \mathrm{~min}$ for racemic sample of $\mathbf{4 d}$.

ee $>99 \%$ 


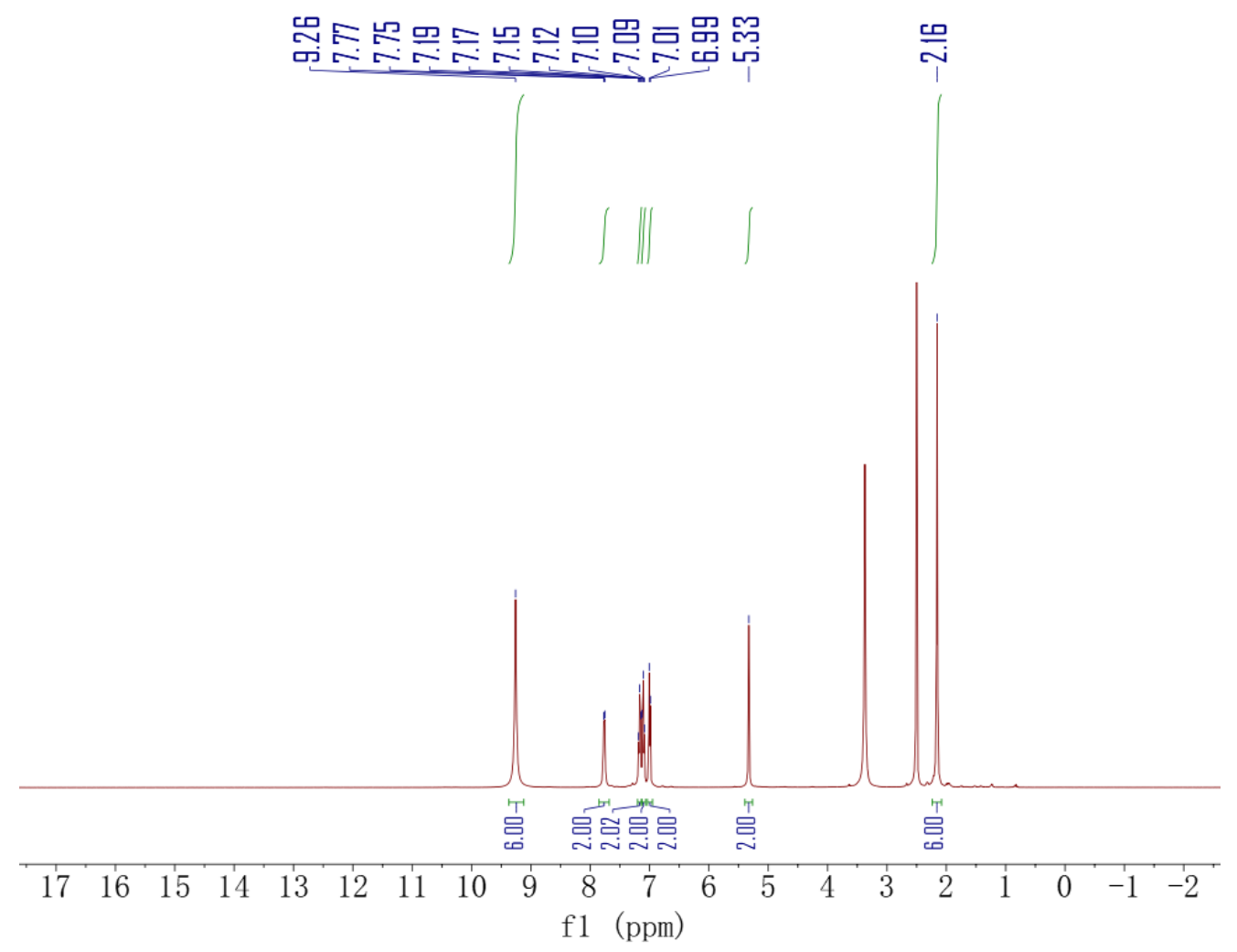

Figure S11. ${ }^{1} \mathrm{H}$ NMR of $\mathbf{4 d}$.

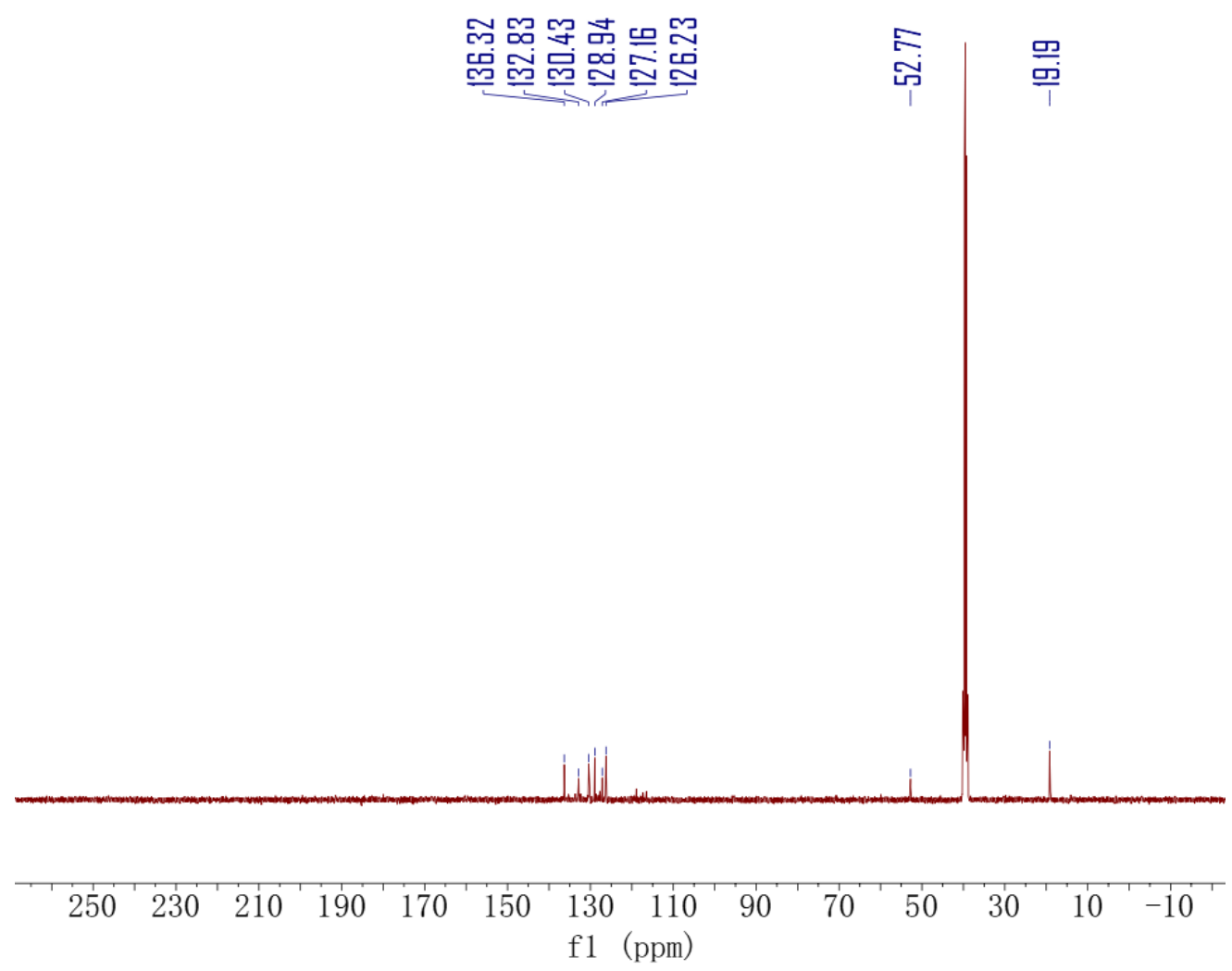

Figure S12. ${ }^{13} \mathrm{C}\left\{{ }^{1} \mathrm{H}\right\}$ NMR of $\mathbf{4 d}$. 

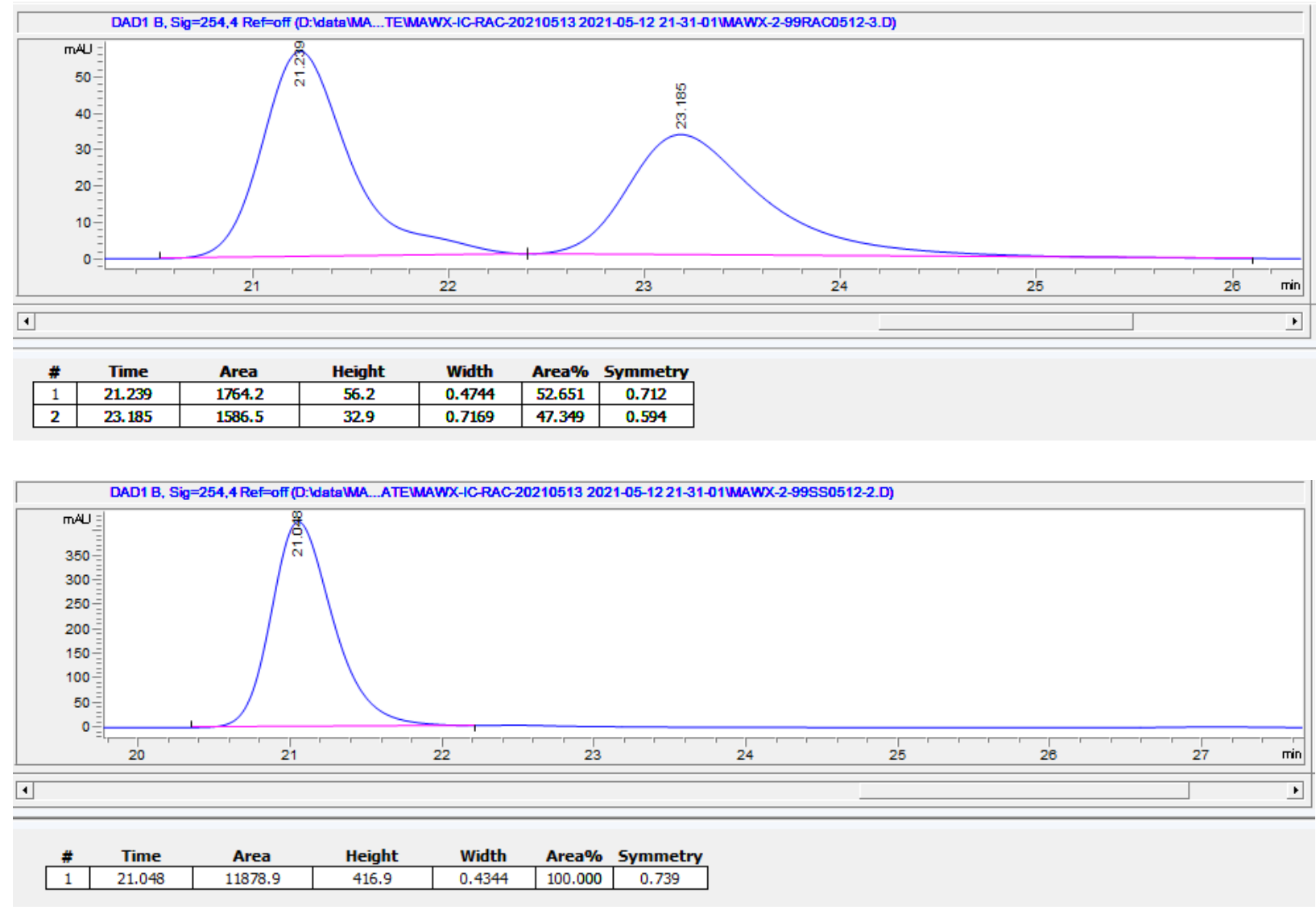

Figure S13. HPLC analysis of (top) racemic sample, and (bottom) $(S, S)$ sample of $4 \mathbf{d}$. 


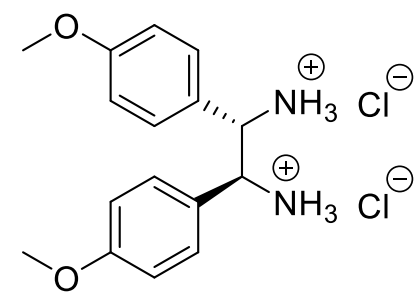

Prepared according to general procedure from $1(50 \mathrm{mg}, 0.20 \mathrm{mmol})$ and 4-

methoxybenzaldehyde (2e) $(58 \mathrm{mg}, 0.43 \mathrm{mmol})$; washed with DCM, EA and THF to give the title compound as a white solid (65 $\mathrm{mg}, 92 \%)$.

${ }^{1} \mathrm{H}$ NMR (DMSO- $\left.d_{6}, 400 \mathrm{MHz}, 25^{\circ} \mathrm{C}\right): \delta 9.22(\mathrm{~s}, 6 \mathrm{H}), 7.27(\mathrm{~d}, J=8.3 \mathrm{~Hz}, 4 \mathrm{H}), 6.81(\mathrm{~d}, J=8.3$ $\mathrm{Hz}, 4 \mathrm{H}), 5.01$ (s, $2 \mathrm{H}), 3.68$ ( s, $6 \mathrm{H})$.

${ }^{13} \mathrm{C}\left\{{ }^{1} \mathrm{H}\right\}$ NMR (DMSO- $\left.d_{6}, 101 \mathrm{MHz}, 25{ }^{\circ} \mathrm{C}\right) \delta 159.42,130.16,125.31,113.80,56.31,55.17$.

HRMS (ESI) calculated for $\mathrm{C}_{16} \mathrm{H}_{21} \mathrm{~N}_{2} \mathrm{O}_{2}$, $[\mathrm{M}+\mathrm{H}]^{+}:$: 273.1598, Found: 273.1589 .

HPLC analysis condition:

Solvent: $n$-hexane/ iso-propanol $=90: 10$

Column: CHIRALPAK IC column (Nomura Chemical Co., Ltd.; inner diameter $4.6 \mathrm{~mm} \times$ length $250 \mathrm{~mm}$; particle size $=5 \mu \mathrm{m}$ )

Column temperature: $25{ }^{\circ} \mathrm{C}$

Detector: UV at $350 \mathrm{~nm}$

Flow rate: $1.0 \mathrm{~mL} / \mathrm{min}$

Injection volume: $5 \mu \mathrm{L}$

$(R, R)-4 \mathbf{e} t_{\mathrm{R}}=8.1 \mathrm{~min},(S, S)-\mathbf{4 e} \mathrm{t}_{\mathrm{R}}=9.8 \mathrm{~min}$ for racemic sample of $\mathbf{4 e}$.

ee $>99 \%$ 


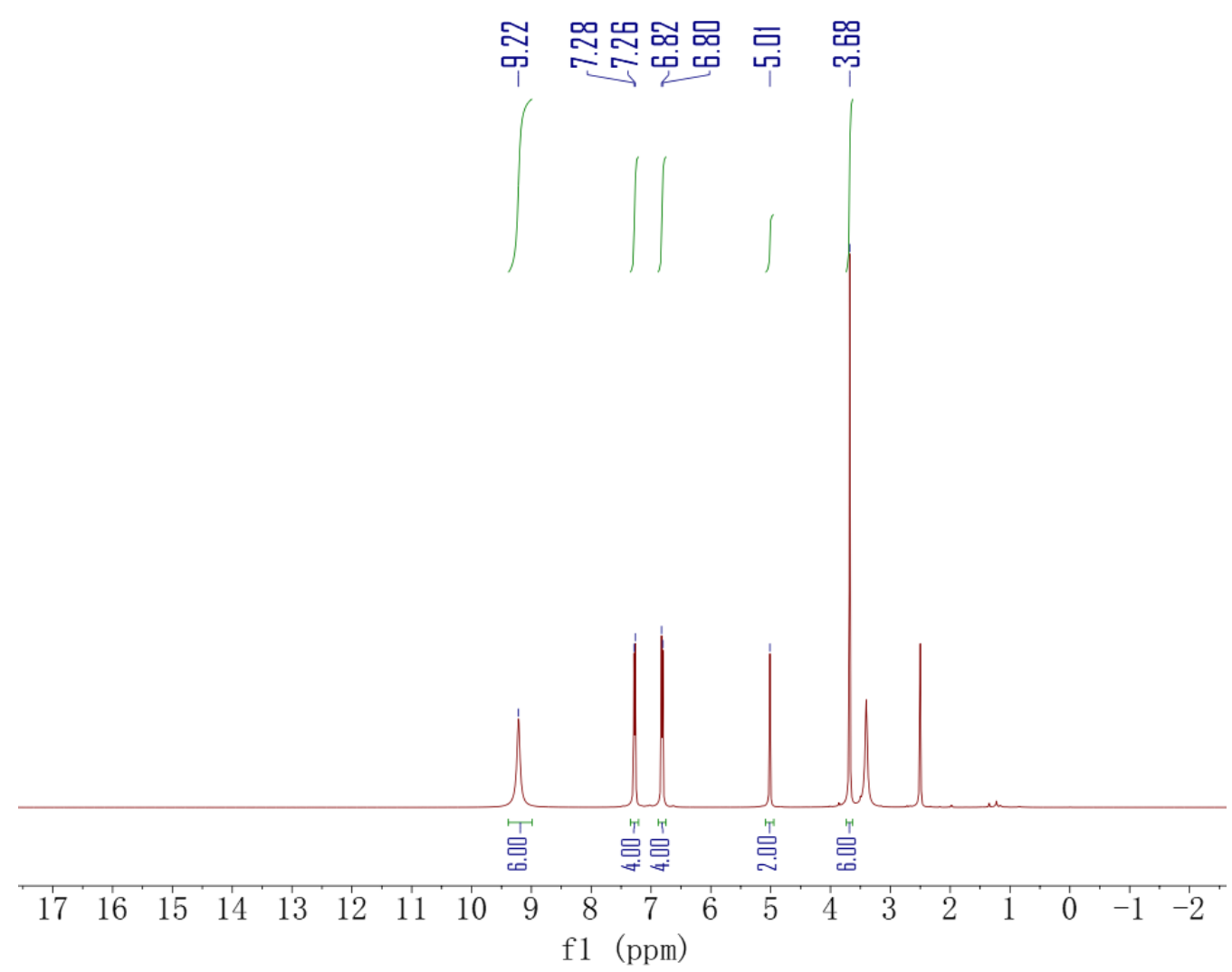

Figure S14. ${ }^{1} \mathrm{H}$ NMR of $4 e$.

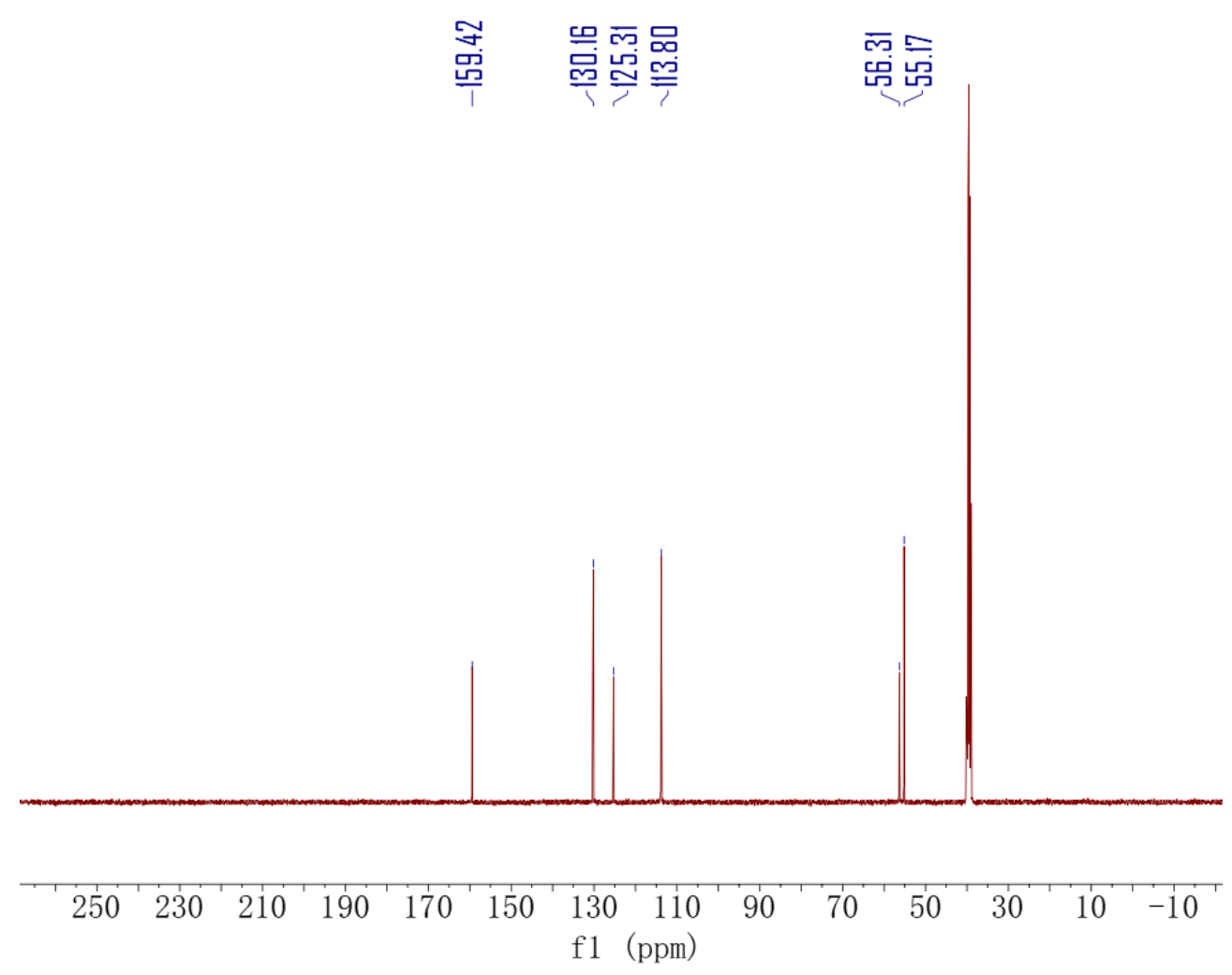

Figure S15. ${ }^{13} \mathrm{C}$ NMR of $4 \mathrm{e}$. 

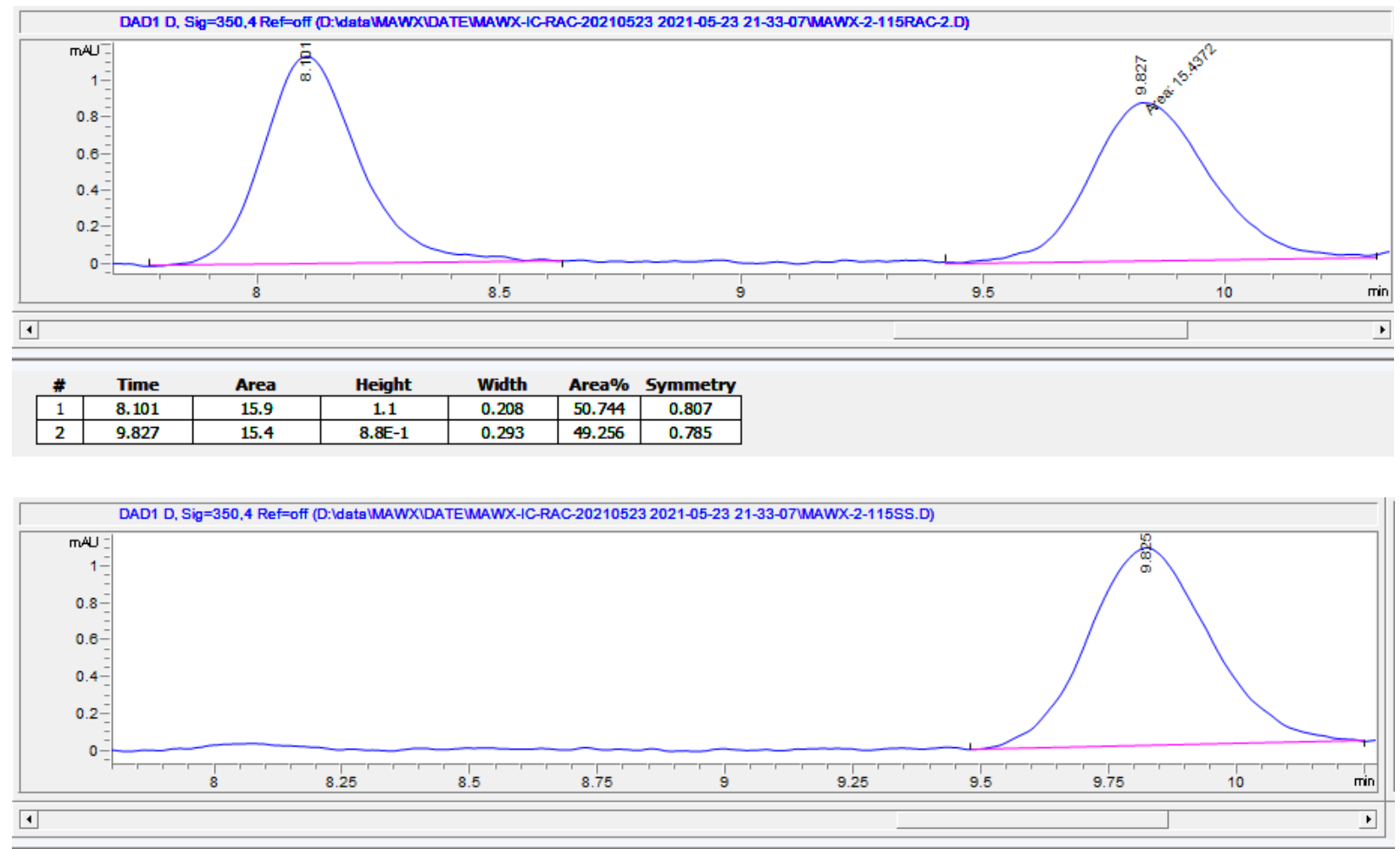

\begin{tabular}{|c|c|c|c|c|c|c|}
\multicolumn{1}{l}{ \# } & Time & Area & \multicolumn{1}{c}{ Height } & Width & \multicolumn{1}{c}{ Area\% } & Symmetry \\
\hline 1 & 9.825 & 18.1 & 1.1 & 0.2485 & 100.000 & 0.905 \\
\hline
\end{tabular}

Figure S16. HPLC analysis of (top) racemic sample, and (bottom) $(S, S)$ sample of $\mathbf{4 e .}$ 


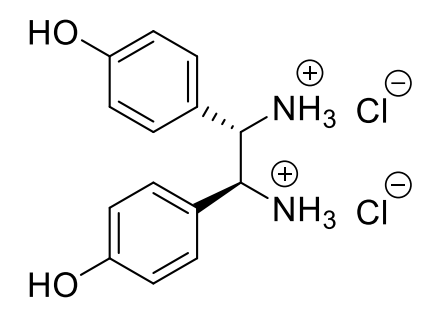

Prepared according to general procedure from 1 (50 $\mathrm{mg}, 0.20 \mathrm{mmol})$ and 4-

hydroxybenzaldehyde (2f) (53 mg, $0.43 \mathrm{mmol}$ ); washed with DCM, EA and THF to give the title compound as a white solid (52 $\mathrm{mg}, 80 \%)$.

${ }^{1} \mathrm{H}$ NMR (DMSO- $\left.d_{6}, 400 \mathrm{MHz}, 25^{\circ} \mathrm{C}\right): \delta 9.73$ (s, $\left.2 \mathrm{H}\right), 9.09(\mathrm{~s}, 6 \mathrm{H}), 7.09(\mathrm{~d}, J=8.2 \mathrm{~Hz}, 4 \mathrm{H})$, $6.65(\mathrm{~d}, J=8.1 \mathrm{~Hz}, 4 \mathrm{H}), 4.87(\mathrm{~s}, 2 \mathrm{H})$.

${ }^{13} \mathrm{C}\left\{{ }^{1} \mathrm{H}\right\}$ NMR (DMSO- $\left.d 6,101 \mathrm{MHz}, 25{ }^{\circ} \mathrm{C}\right) \delta 157.90,130.03,123.35,115.22,56.52$.

HRMS (ESI) calculated for $\mathrm{C}_{14} \mathrm{H}_{17} \mathrm{~N}_{2} \mathrm{O}_{2},[\mathrm{M}+\mathrm{H}]^{+}:$: 245.1285, Found: 245.1275.

HPLC analysis condition:

Solvent: $n$-hexane/ iso-propanol $=90: 10$

Column: CHIRALPAK IC column (Nomura Chemical Co., Ltd.; inner diameter $4.6 \mathrm{~mm} \times$ length $250 \mathrm{~mm}$; particle size $=5 \mu \mathrm{m}$ )

Column temperature: $25^{\circ} \mathrm{C}$

Detector: UV at $254 \mathrm{~nm}$

Flow rate: $0.8 \mathrm{~mL} / \mathrm{min}$

Injection volume: $5 \mu \mathrm{L}$

$(R, R)-\mathbf{4 f} \mathrm{t}_{\mathrm{R}}=19.6 \mathrm{~min},(S, S)-\mathbf{4 f} \mathrm{t}_{\mathrm{R}}=22.0 \mathrm{~min}$ for racemic sample of $\mathbf{4 f}$.

ee $>99 \%$ 


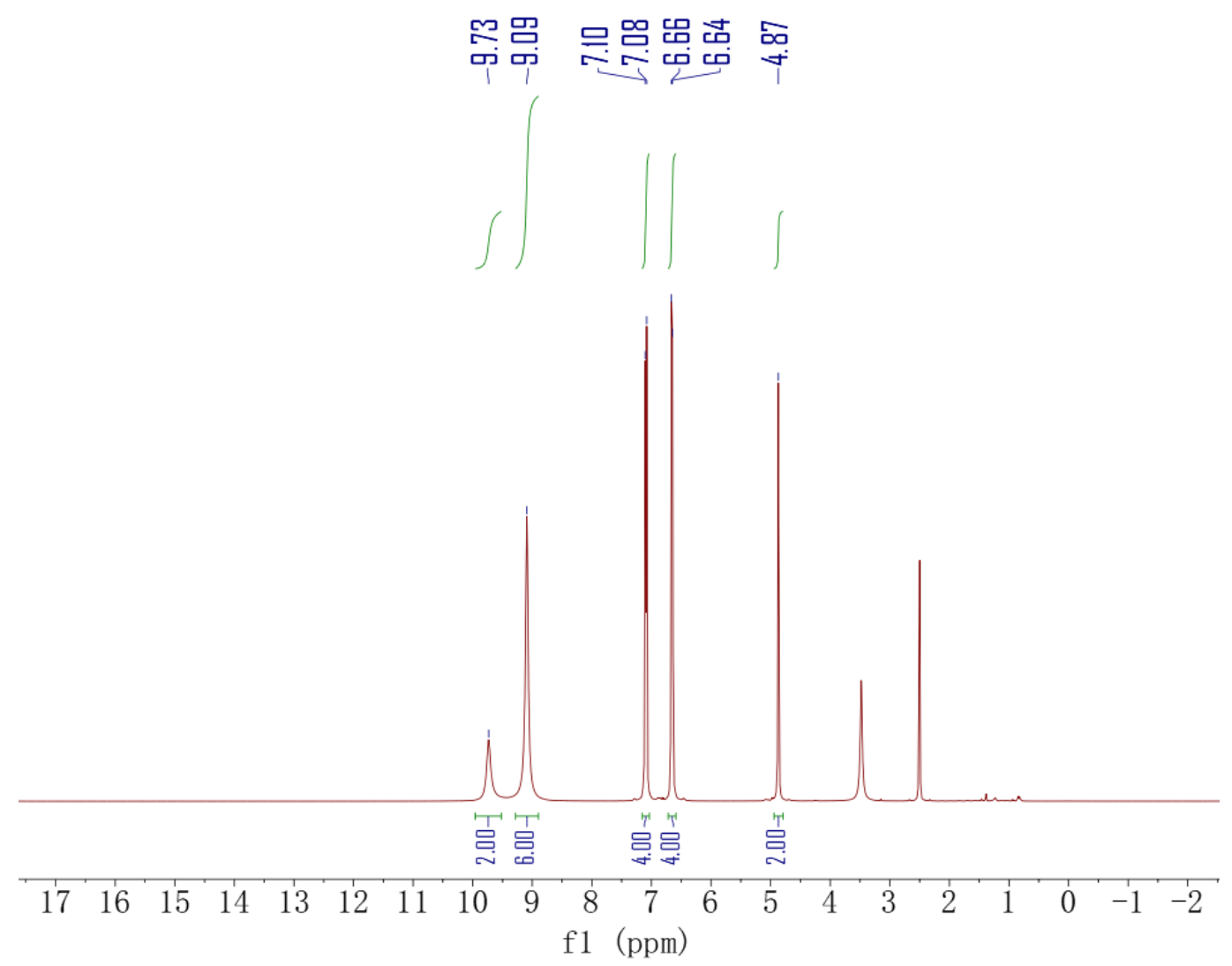

Figure S17. ${ }^{1} \mathrm{H}$ NMR of $\mathbf{4 f}$.
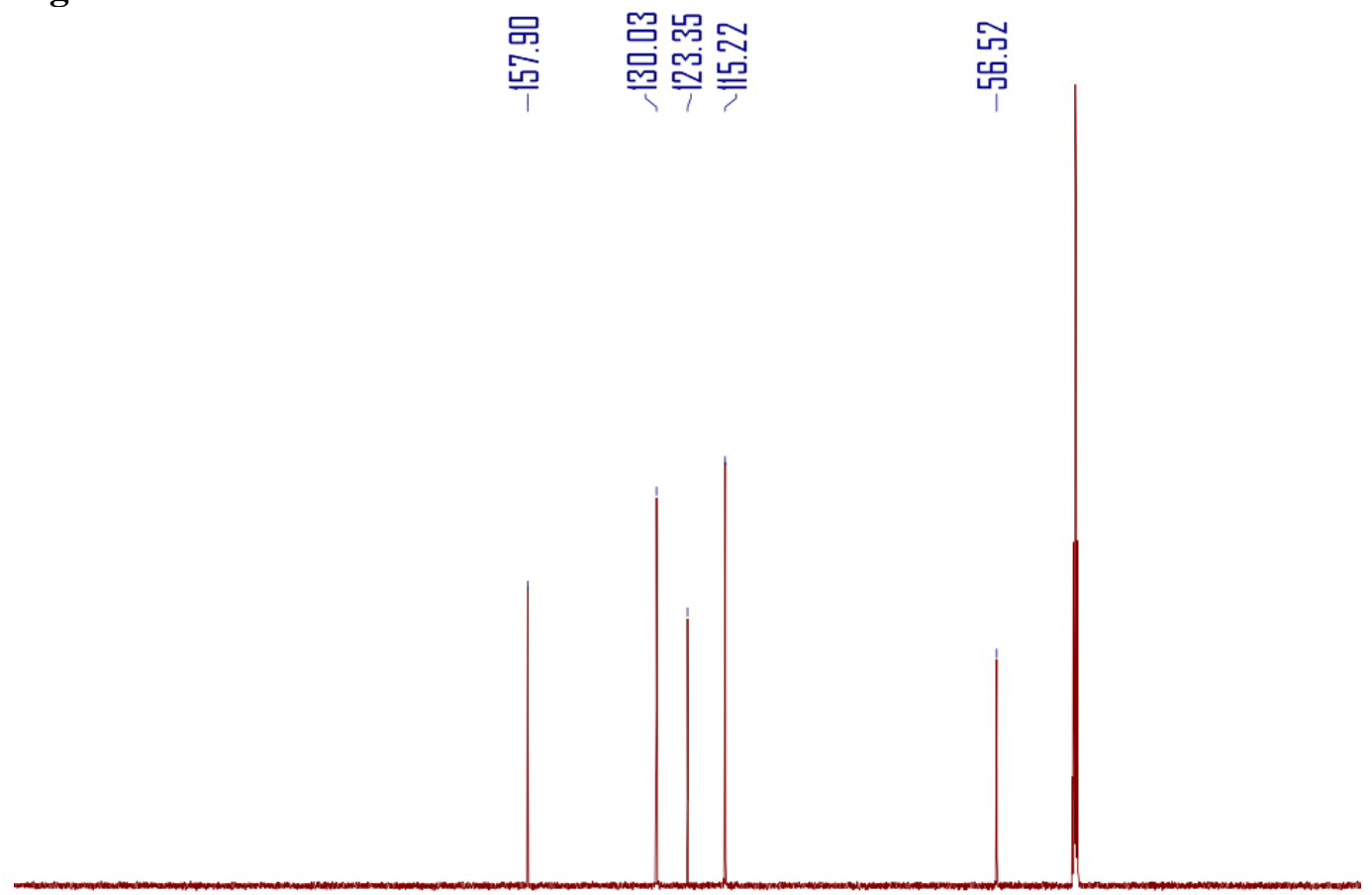

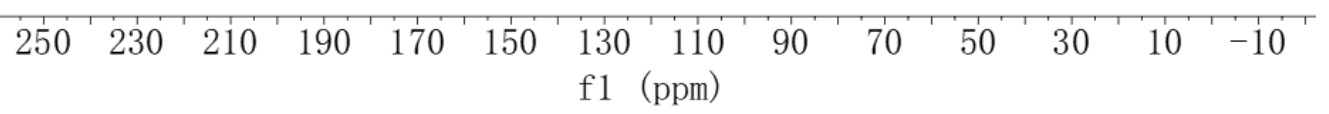

Figure S18. ${ }^{13} \mathrm{C}\left\{{ }^{1} \mathrm{H}\right\}$ NMR of $\mathbf{4 f}$. 


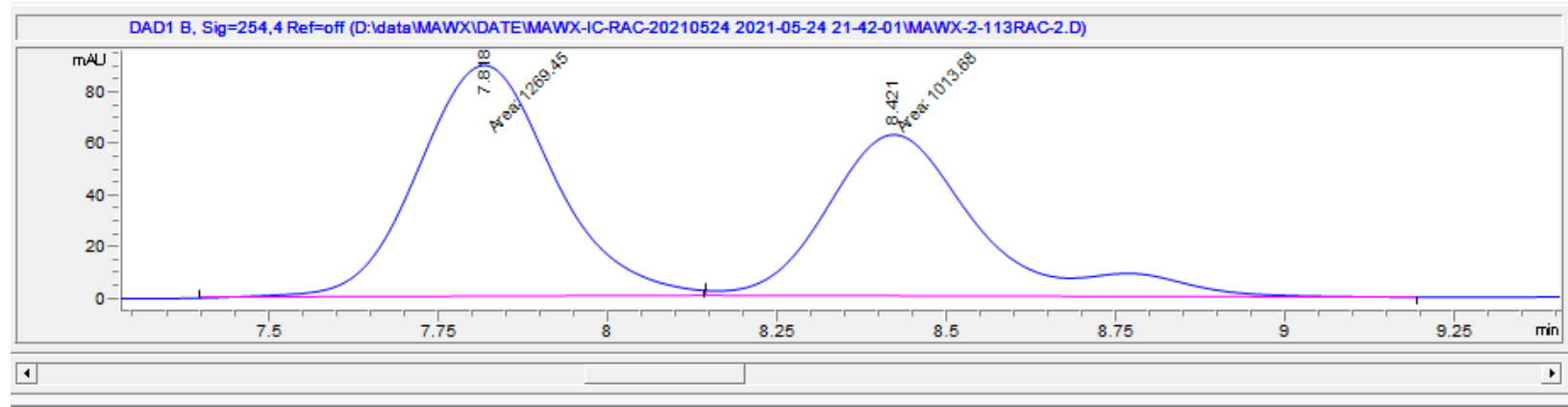

\begin{tabular}{|c|c|c|c|c|c|c|}
\hline \# & Time & Area & Height & Width & Area $\%$ & Symmetry \\
\hline 1 & 7.818 & 1269.4 & 90.8 & 0.2329 & 55.601 & 0.93 \\
\hline 2 & 8.421 & 1013.7 & 63.5 & 0.2659 & 44.399 & 0.729 \\
\hline
\end{tabular}

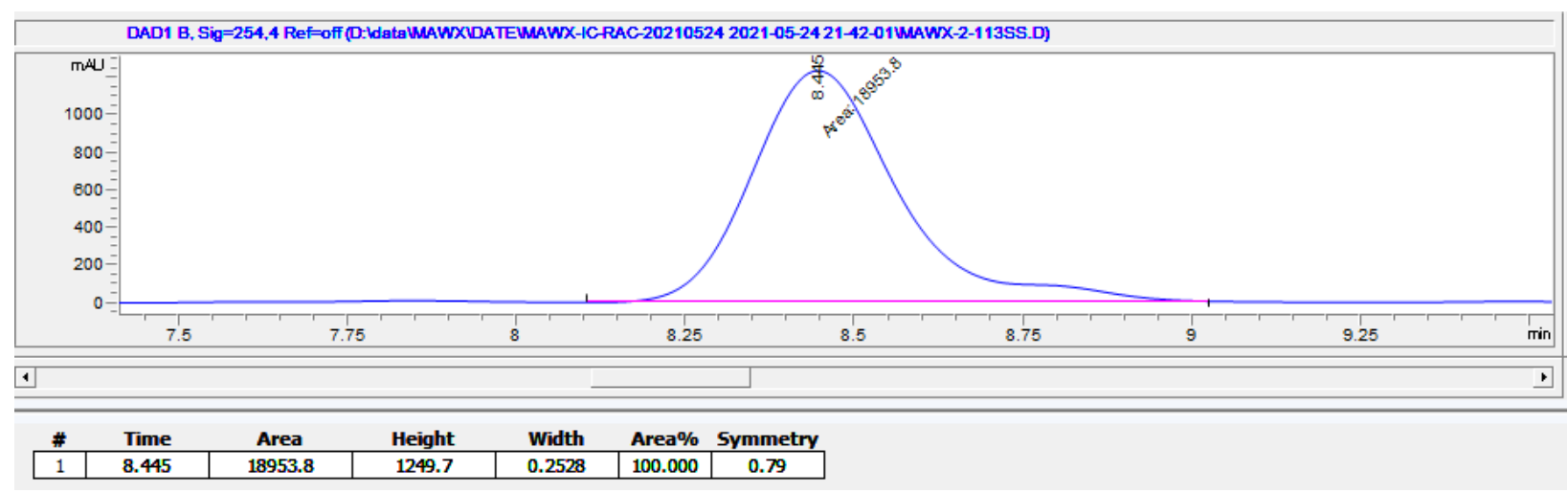

Figure S19. HPLC analysis of (top) racemic sample, and (bottom) $(S, S)$ sample of $\mathbf{4 f}$. 


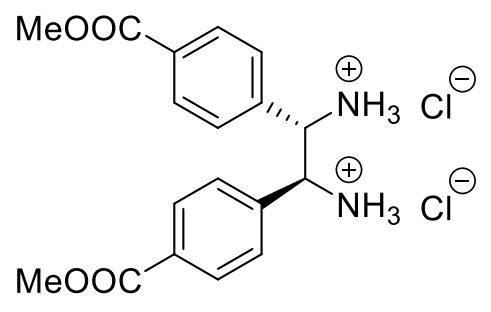

Prepared according to general procedure from 1 (50 mg, $0.20 \mathrm{mmol})$ and methyl 4-

formylbenzoate $(\mathbf{2 g})$ (70 $\mathrm{mg}, 0.43 \mathrm{mmol})$; washed with DCM, EA and THF to give the title compound as a white solid (66 mg, 81\%).

${ }^{1} \mathrm{H}$ NMR (DMSO- $\left.d_{6}, 500 \mathrm{MHz}, 25^{\circ} \mathrm{C}\right): \delta 9.48(\mathrm{~s}, 6 \mathrm{H}), 7.80(\mathrm{~d}, J=8.2 \mathrm{~Hz}, 4 \mathrm{H}), 7.54(\mathrm{~d}, J=8.3$ $\mathrm{Hz}, 4 \mathrm{H}), 5.25$ (s, 2H), 3.80 (s, $6 \mathrm{H})$.

${ }^{13} \mathrm{C}\left\{{ }^{1} \mathrm{H}\right\}$ NMR (DMSO- $\left.d_{6}, 101 \mathrm{MHz}, 25{ }^{\circ} \mathrm{C}\right) \delta 165.61,138.20,130.05,129.27,129.16,56.38$, 52.31 .

HRMS (ESI) calculated for $\mathrm{C}_{18} \mathrm{H}_{21} \mathrm{~N}_{2} \mathrm{O}_{4},[\mathrm{M}+\mathrm{H}]^{+}: 329.1496$, Found: 329.1486.

HPLC analysis condition:

Solvent: n-hexane/ iso-propanol $=96: 4$

Column: CHIRALPAK IC column (Nomura Chemical Co., Ltd.; inner diameter $4.6 \mathrm{~mm} \times$ length $250 \mathrm{~mm}$; particle size $=5 \mu \mathrm{m}$ )

Column temperature: $25^{\circ} \mathrm{C}$

Detector: UV at $254 \mathrm{~nm}$

Flow rate: $0.7 \mathrm{~mL} / \mathrm{min}$

Injection volume: $5 \mu \mathrm{L}$

$(R, R)-\mathbf{4} \mathbf{g} \mathrm{t}_{\mathrm{R}}=7.8 \mathrm{~min},(S, S) \mathbf{- 4} \mathbf{g} \mathrm{t}_{\mathrm{R}}=8.4 \mathrm{~min}$ for racemic sample of $\mathbf{4 g}$.

ee $>99 \%$ 


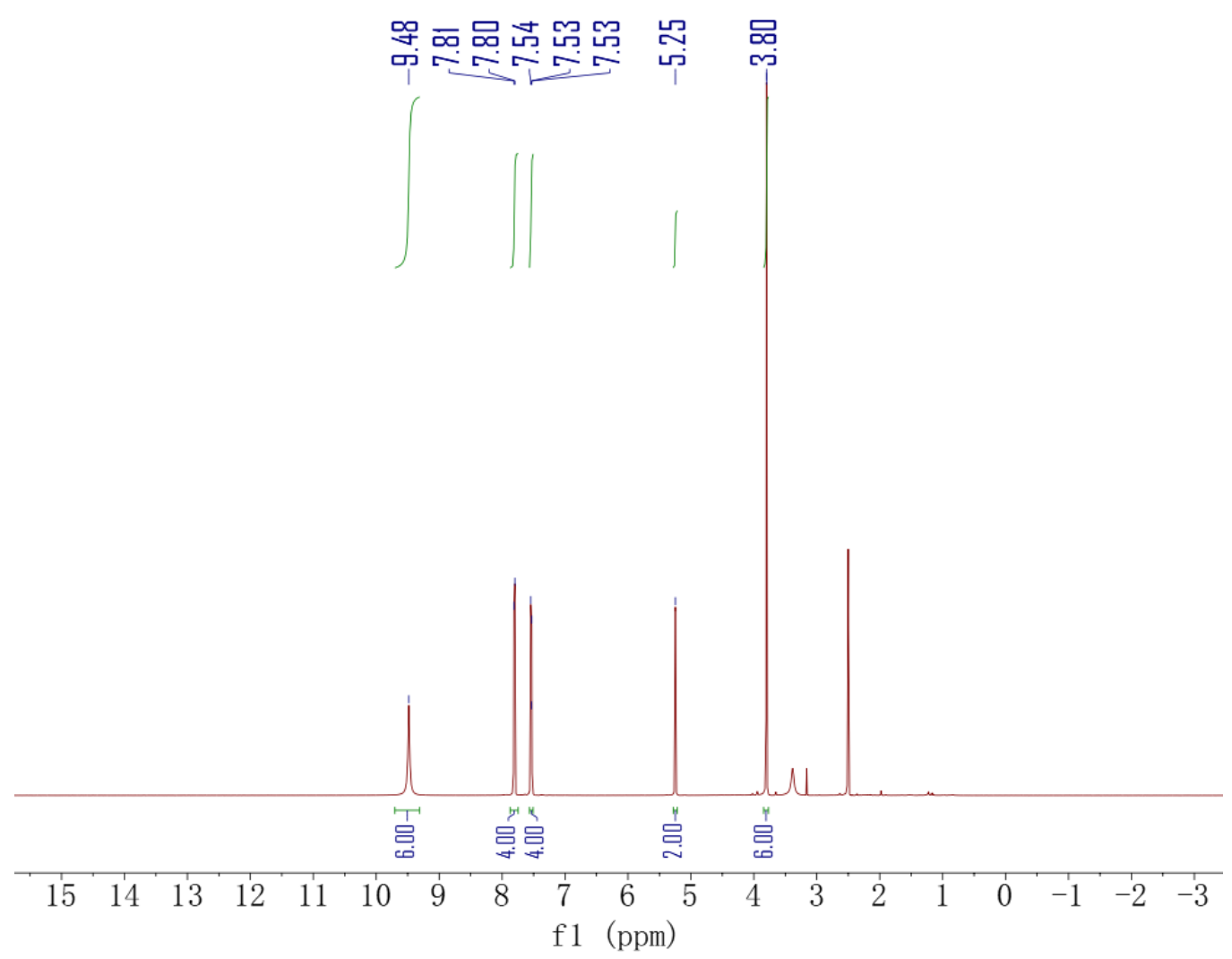

Figure S20. ${ }^{1} \mathrm{H}$ NMR of $\mathbf{4 g}$.

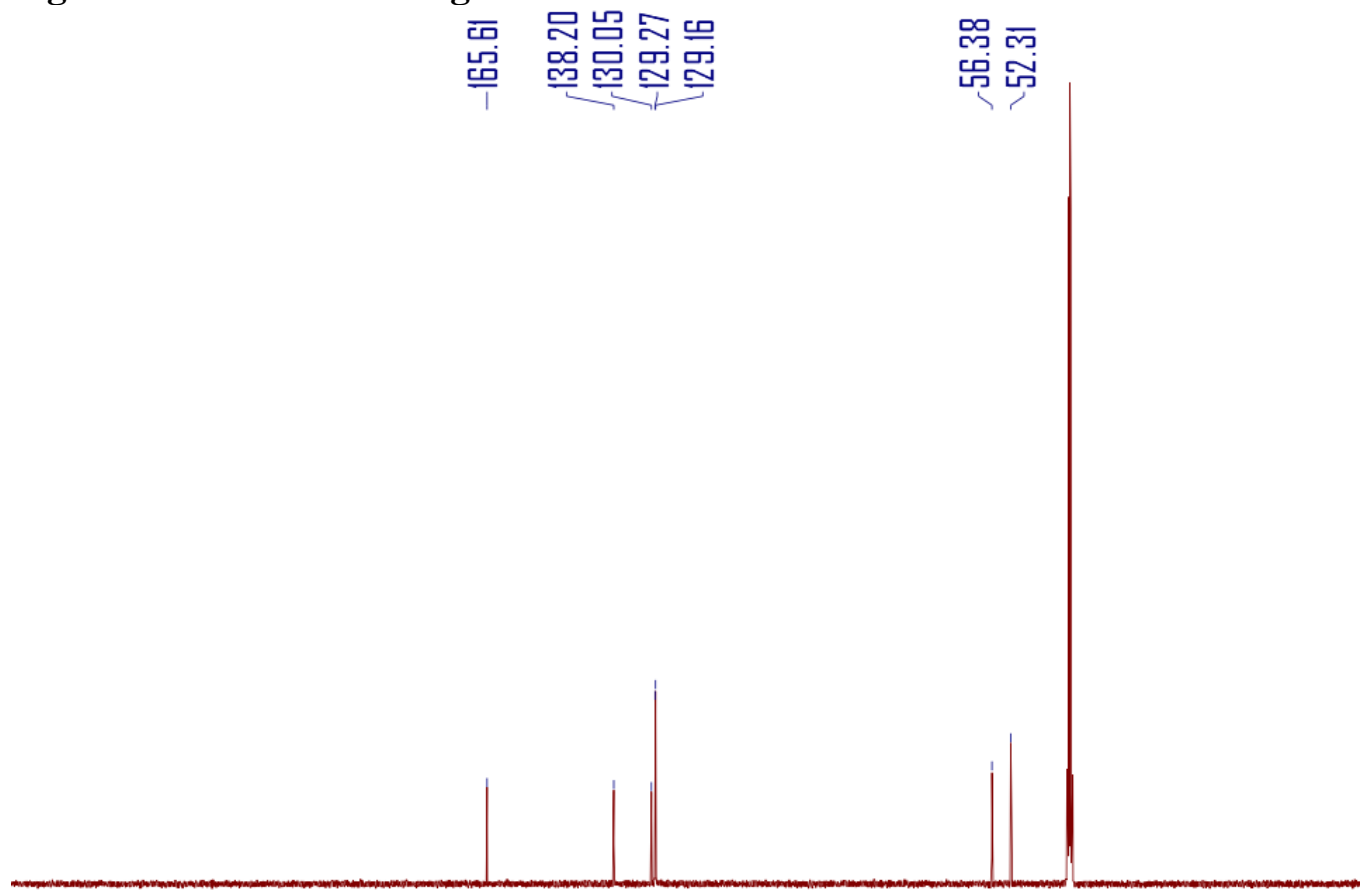

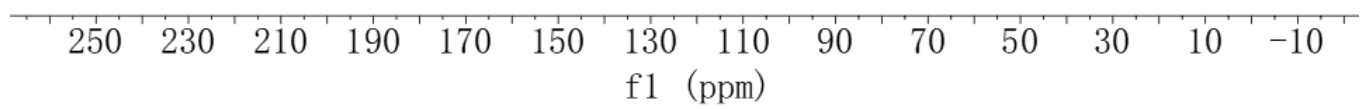

Figure S21. ${ }^{13} \mathrm{C}\left\{{ }^{1} \mathrm{H}\right\}$ NMR of $\mathbf{4 g}$. 


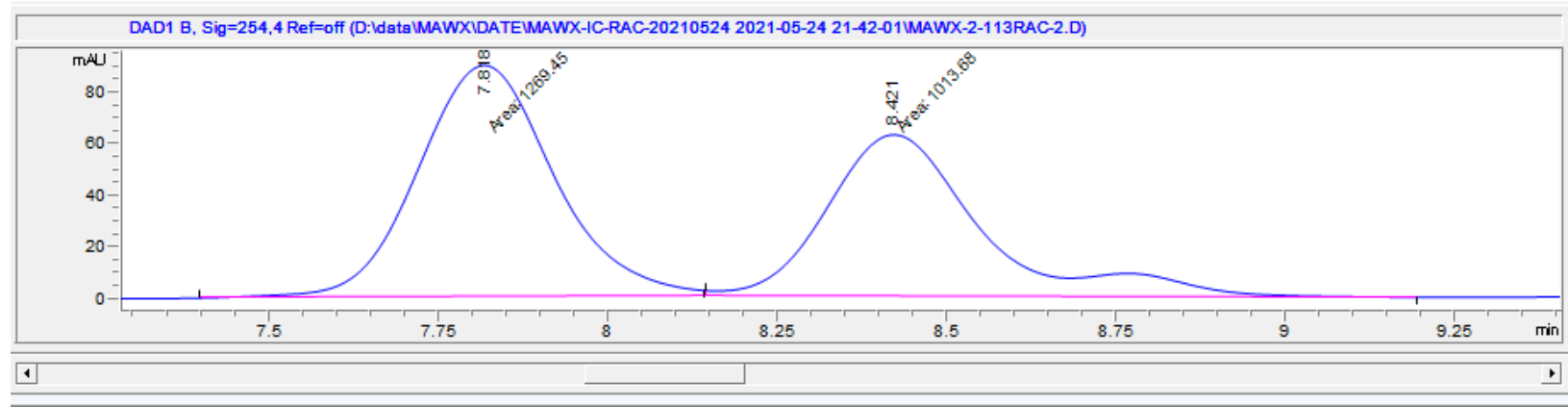

\begin{tabular}{|c|c|c|c|c|c|c|}
\hline \# & Time & Area & Height & Width & Area $\%$ & Symmetry \\
\hline 1 & 7.818 & 1269.4 & 90.8 & 0.2329 & 55.601 & 0.93 \\
\hline 2 & 8.421 & 1013.7 & 63.5 & 0.2659 & 44.399 & 0.729 \\
\hline
\end{tabular}

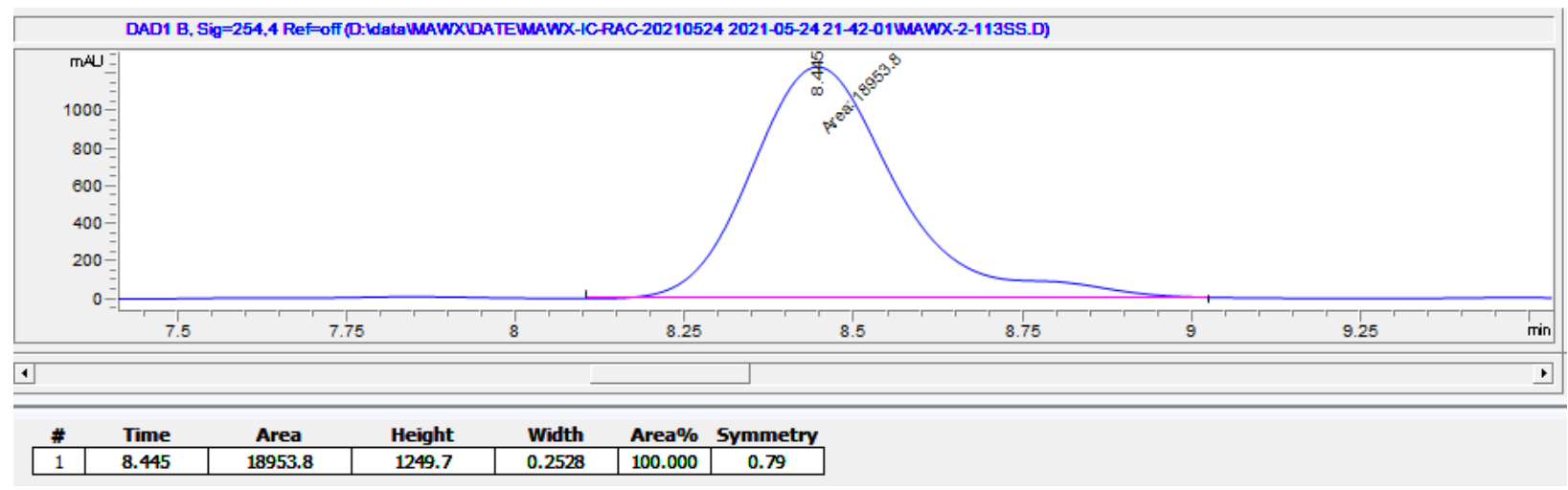

Figure S22. HPLC analysis of (top) racemic sample, and (bottom) $(S, S)$ sample of $\mathbf{4 g}$. 


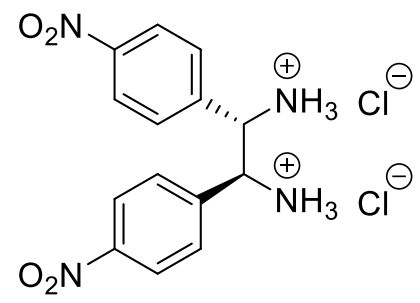

Prepared according to general procedure from 1 (50 $\mathrm{mg}, 0.20 \mathrm{mmol})$ and 4-nitrobenzaldehyde (2h) (65 mg, $0.43 \mathrm{mmol})$; washed with DCM, EA and THF to give the title compound as a white solid (61 mg, 79\%).

${ }^{1} \mathrm{H}$ NMR (DMSO- $\left.d_{6}, 400 \mathrm{MHz}, 25{ }^{\circ} \mathrm{C}\right): \delta 9.46(\mathrm{~s}, 6 \mathrm{H}), 8.17(\mathrm{~d}, J=8.4 \mathrm{~Hz}, 4 \mathrm{H}), 7.72(\mathrm{~d}, J=8.4$ $\mathrm{Hz}, 4 \mathrm{H}), 5.36$ ( $\mathrm{s}, 2 \mathrm{H})$.

${ }^{13} \mathrm{C}\left\{{ }^{1} \mathrm{H}\right\}$ NMR (DMSO- $\left.d_{6}, 101 \mathrm{MHz}, 25{ }^{\circ} \mathrm{C}\right) \delta 147.76,139.96,130.52,123.60,55.76$.

HRMS (ESI) calculated for $\mathrm{C}_{14} \mathrm{H}_{15} \mathrm{~N}_{4} \mathrm{O}_{4},[\mathrm{M}+\mathrm{H}]^{+}: 303.1088$, Found: 303.1076.

HPLC analysis condition:

Solvent: $\mathrm{n}$-hexane/ iso-propanol $=95: 5$

Column: CHIRALPAK IC column (Nomura Chemical Co., Ltd.; inner diameter $4.6 \mathrm{~mm} \times$ length $250 \mathrm{~mm}$; particle size $=5 \mu \mathrm{m}$ )

Column temperature: $25{ }^{\circ} \mathrm{C}$

Detector: UV at $350 \mathrm{~nm}$

Flow rate: $1.0 \mathrm{~mL} / \mathrm{min}$

Injection volume: $5 \mu \mathrm{L}$

$(R, R)-\mathbf{4 h} \mathrm{t}_{\mathrm{R}}=14.9 \mathrm{~min},(S, S)-\mathbf{4 h} \mathrm{t}_{\mathrm{R}}=16.4 \mathrm{~min}$ for racemic sample of $\mathbf{4 h}$.

ee $>99 \%$ 


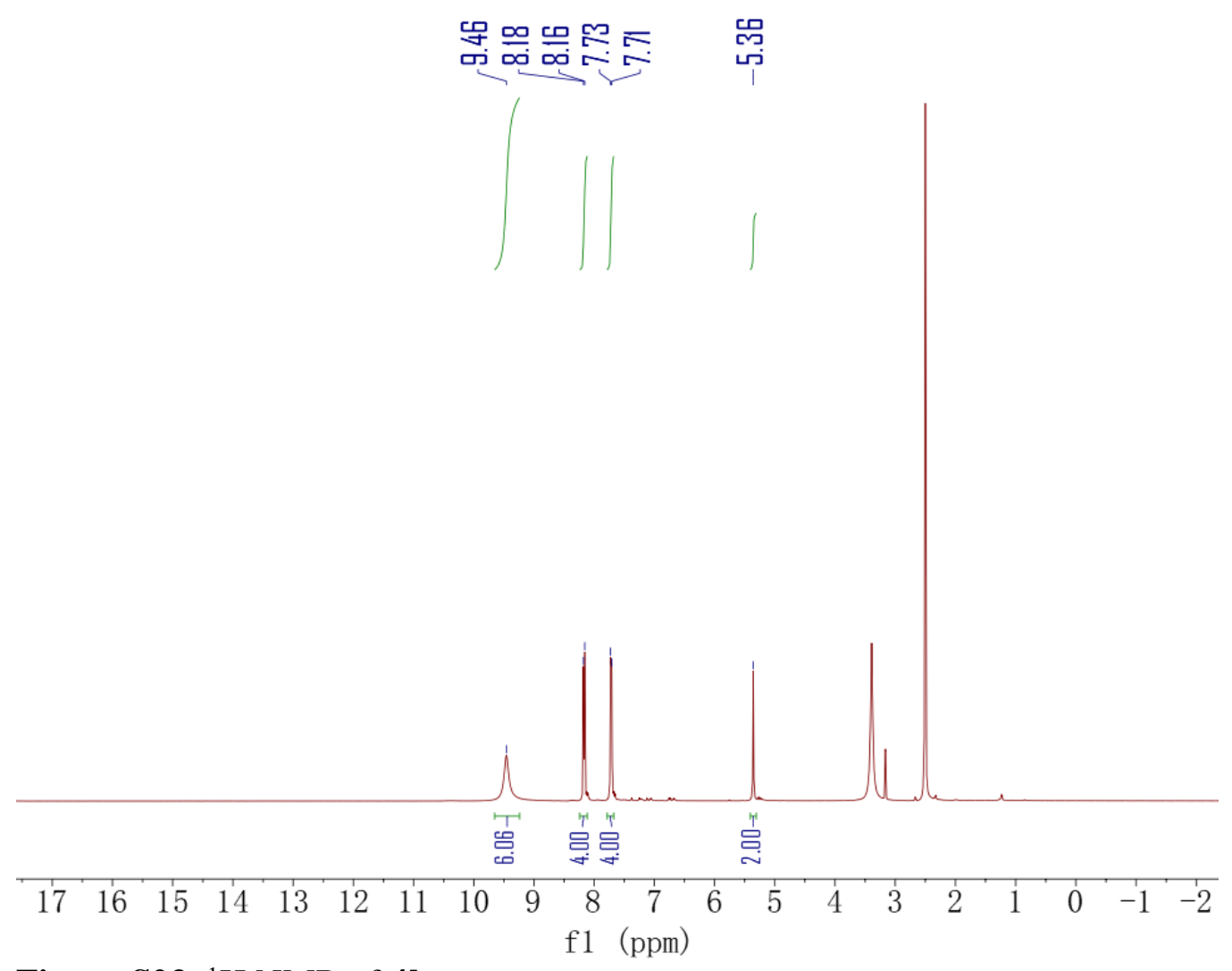

Figure S23. ${ }^{1} \mathrm{H}$ NMR of $\mathbf{4 h}$.

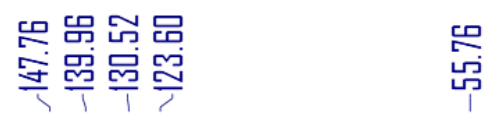

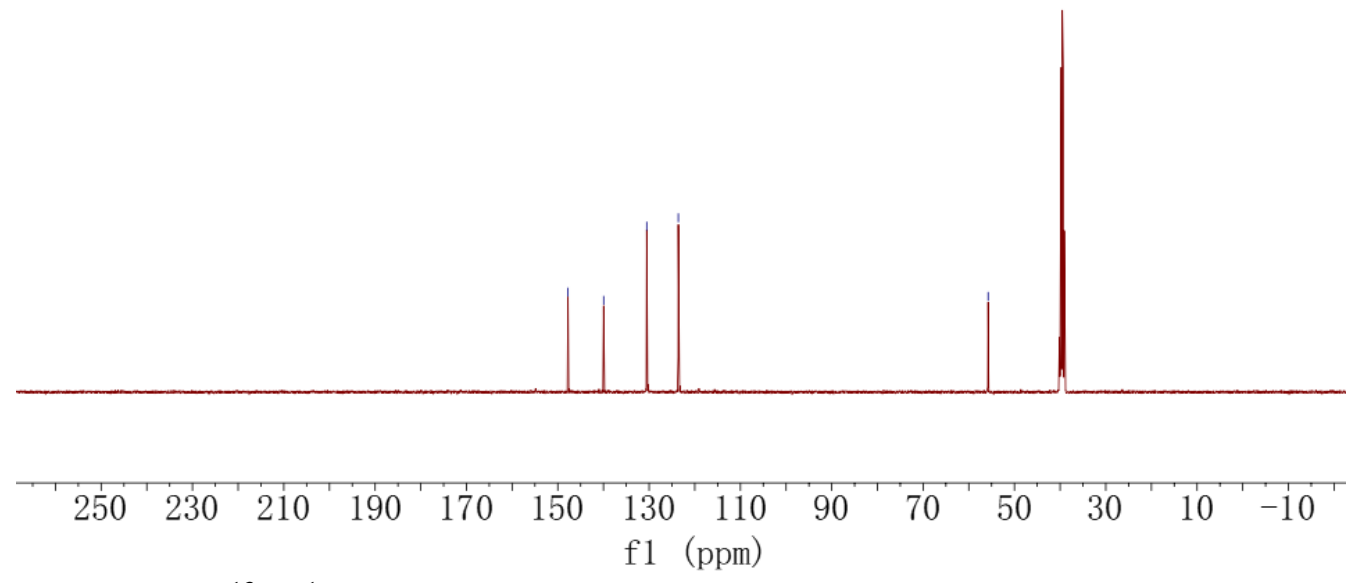

Figure S24. ${ }^{13} \mathrm{C}\left\{{ }^{1} \mathrm{H}\right\}$ NMR of $\mathbf{4 h}$. 

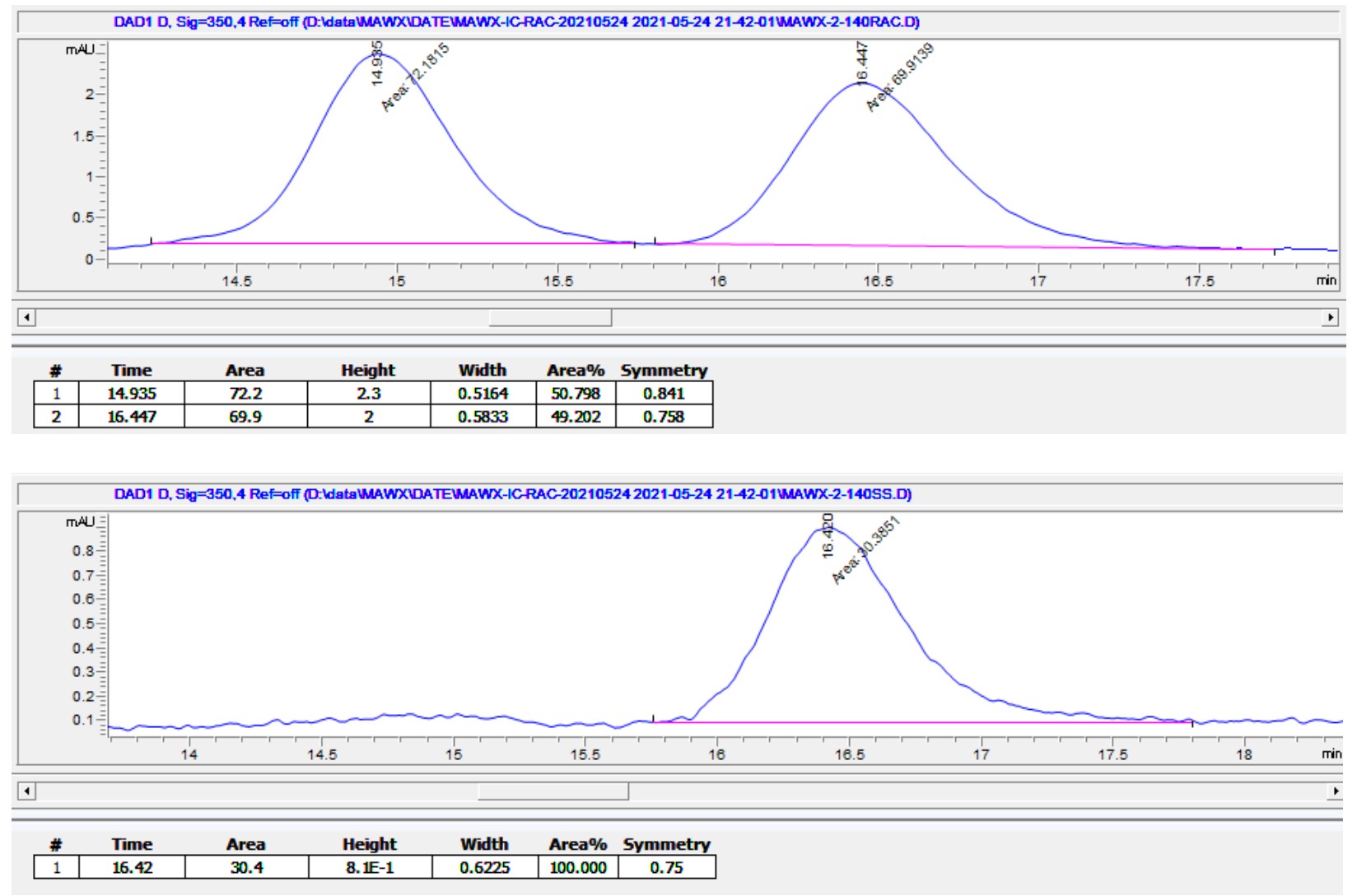

Figure S25. HPLC analysis of (top) racemic sample, and (bottom) $(S, S)$ sample of $\mathbf{4 h}$. 


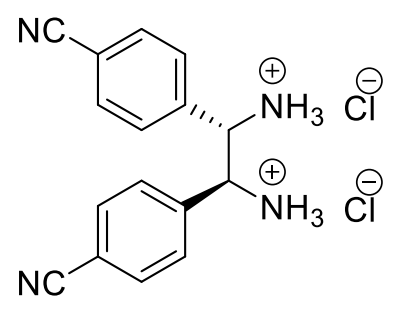

Prepared according to general procedure from 1 (50 $\mathrm{mg}, 0.20 \mathrm{mmol})$ and 4-formylbenzonitrile (2i) (56 mg, $0.43 \mathrm{mmol}$ ); washed with DCM, EA and THF to give the title compound as a white solid (58 $\mathrm{mg}, 85 \%)$.

${ }^{1} \mathrm{H}$ NMR (DMSO- $\left.d_{6}, 400 \mathrm{MHz}, 25^{\circ} \mathrm{C}\right): \delta 9.55(\mathrm{~s}, 6 \mathrm{H}), 7.79(\mathrm{~d}, J=8.0 \mathrm{~Hz}, 4 \mathrm{H}), 7.62(\mathrm{~d}, J=8.0$ $\mathrm{Hz}, 4 \mathrm{H}), 5.31$ ( $\mathrm{s}, 2 \mathrm{H})$.

${ }^{13} \mathrm{C}\left\{{ }^{1} \mathrm{H}\right\}$ NMR (DMSO- $\left.d 6,101 \mathrm{MHz}, 25{ }^{\circ} \mathrm{C}\right) \delta 138.11,132.47,129.92,118.24,111.92,56.01$.

HRMS (ESI) calculated for $\mathrm{C}_{16} \mathrm{H}_{15} \mathrm{~N}_{4},[\mathrm{M}+\mathrm{H}]^{+}:$263.1291, Found: 263.1284.

HPLC analysis condition:

Solvent: $n$-hexane/ iso-propanol $=60: 40$

Column: CHIRALPAK OD column (Nomura Chemical Co., Ltd.; inner diameter $4.6 \mathrm{~mm} \times$ length $250 \mathrm{~mm}$; particle size $=5 \mu \mathrm{m}$ )

Column temperature: $25^{\circ} \mathrm{C}$

Detector: UV at $254 \mathrm{~nm}$

Flow rate: $1.0 \mathrm{~mL} / \mathrm{min}$

Injection volume: $5 \mu \mathrm{L}$

$(S, S)-\mathbf{4 i} t_{\mathrm{R}}=18.9 \mathrm{~min},(R, R)-\mathbf{4 i} \mathrm{t}_{\mathrm{R}}=22.6 \mathrm{~min}$ for racemic sample of $\mathbf{4} \mathbf{i}$.

ee $>99 \%$ 


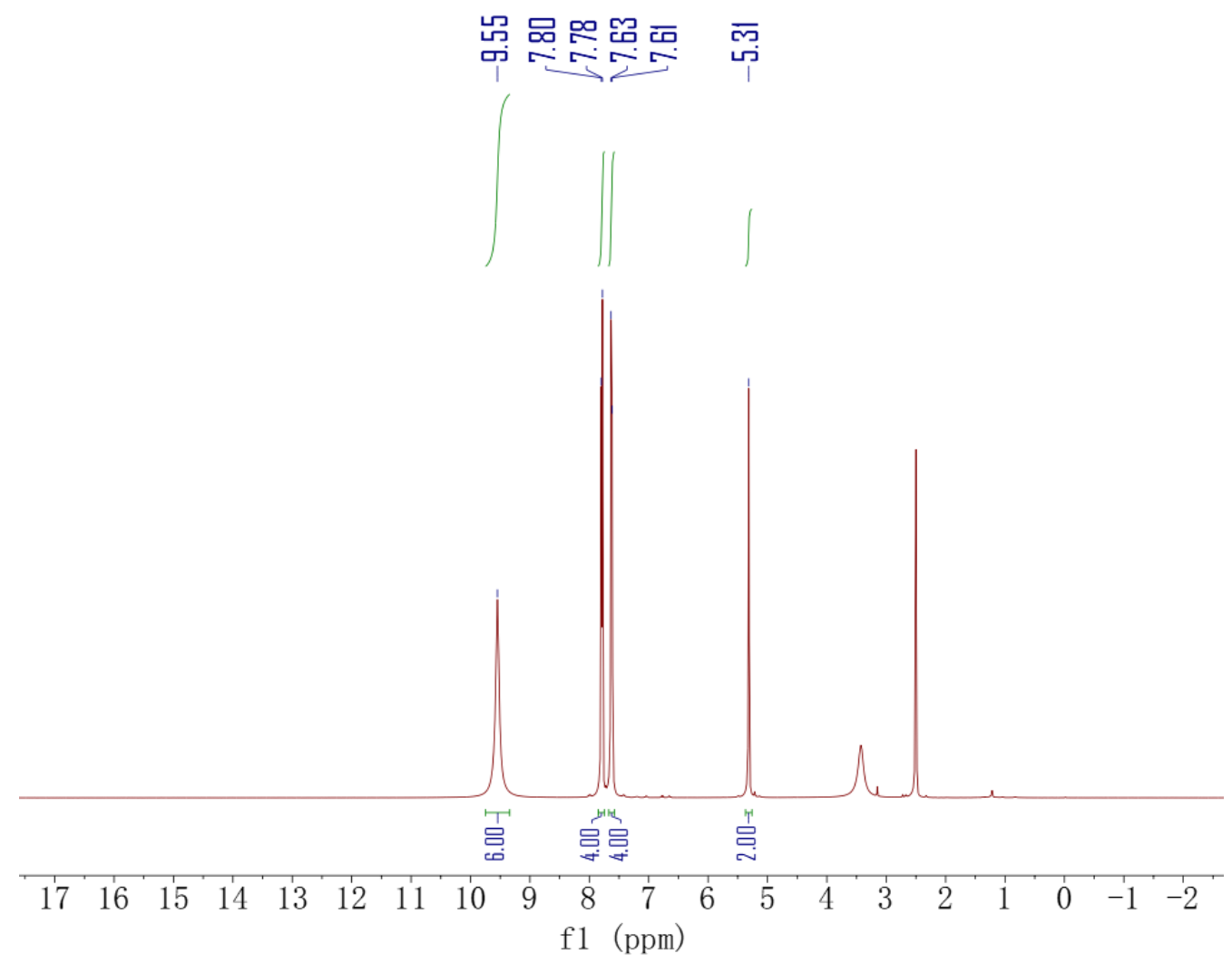

Figure S26. ${ }^{1} \mathrm{H}$ NMR of $4 \mathbf{i}$.

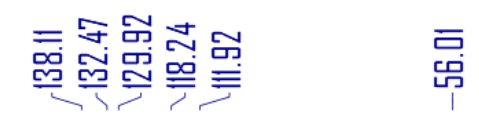

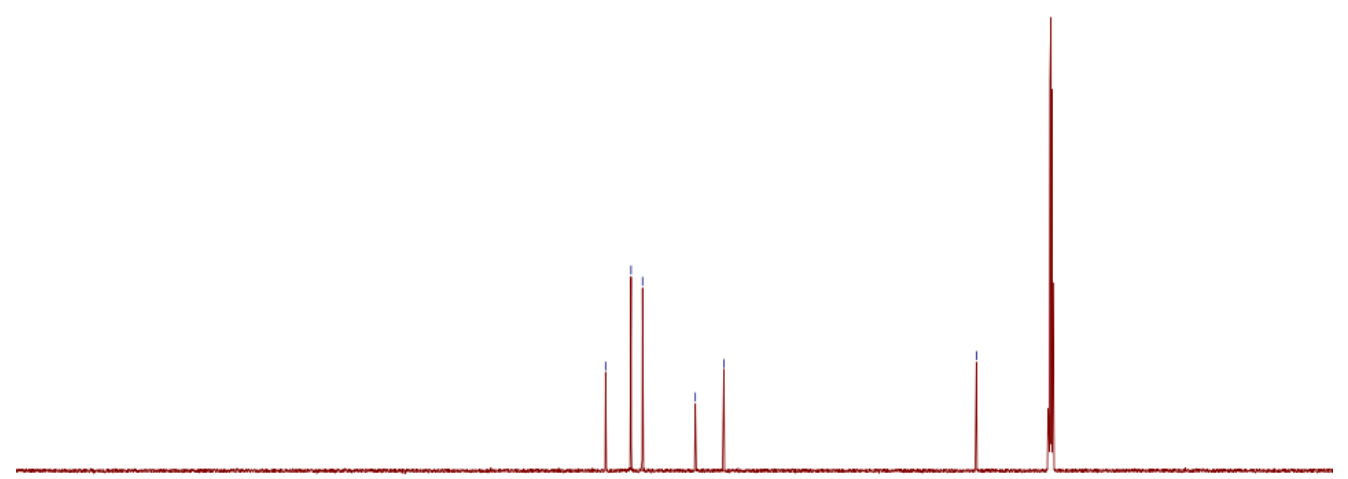

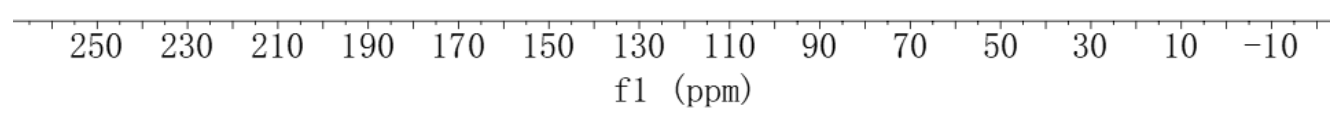

Figure S27. ${ }^{13} \mathrm{C}\left\{{ }^{1} \mathrm{H}\right\}$ NMR of $4 \mathbf{i}$. 

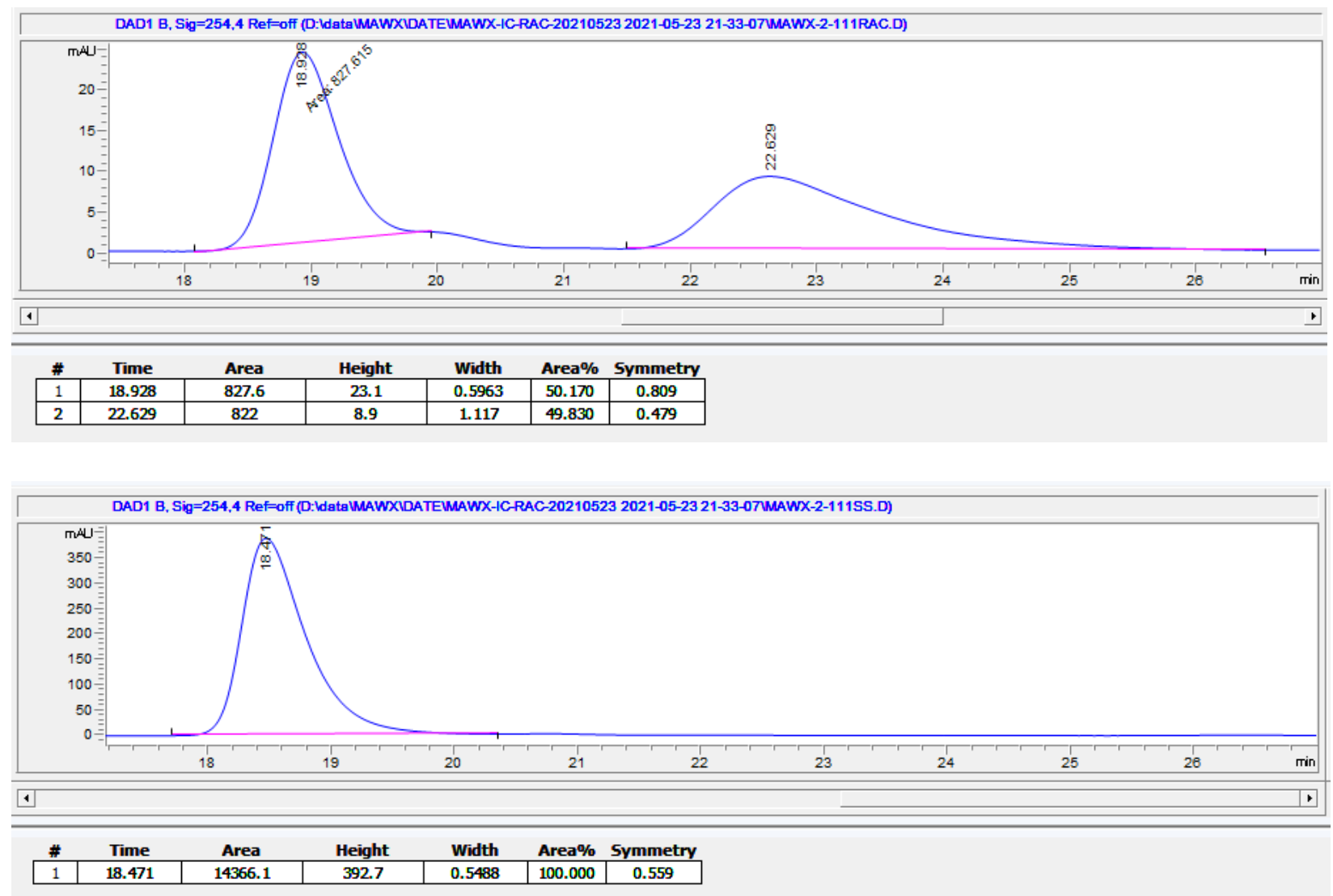

Figure S28. HPLC analysis of (top) racemic sample, and (bottom) $(S, S)$ sample of $\mathbf{4 i}$. 


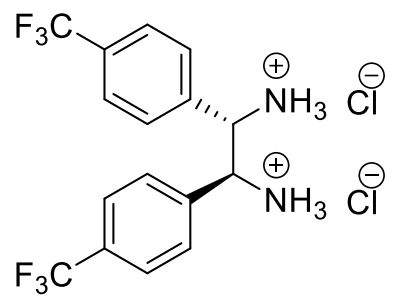

Prepared according to general procedure from $1(50 \mathrm{mg}, 0.20 \mathrm{mmol})$ and 4-

(trifluoromethyl)benzaldehyde (2j) $(75 \mathrm{mg}, 0.43 \mathrm{mmol})$; washed with DCM, EA and THF to give the title compound as a white solid (67 $\mathrm{mg}, 78 \%)$.

${ }^{1} \mathrm{H}$ NMR (DMSO- $\left.d_{6}, 500 \mathrm{MHz}, 25{ }^{\circ} \mathrm{C}\right): \delta 9.50(\mathrm{~s}, 6 \mathrm{H}), 7.66(\mathrm{q}, J=8.2 \mathrm{~Hz}, 8 \mathrm{H}), 5.32(\mathrm{~s}, 2 \mathrm{H})$.

${ }^{13} \mathrm{C}\left\{{ }^{1} \mathrm{H}\right\}$ NMR (DMSO- $\left.d_{6}, 101 \mathrm{MHz}, 25{ }^{\circ} \mathrm{C}\right) \delta 137.53,129.82,125.45,125.41,122.49,56.04$.

${ }^{19} \mathrm{~F}\left\{{ }^{1} \mathrm{H}\right\}$ NMR (DMSO- $\left.d_{6}, 471 \mathrm{MHz}, 25{ }^{\circ} \mathrm{C}\right) \delta-61.38$.

HRMS (ESI) calculated for $\mathrm{C}_{16} \mathrm{H}_{15} \mathrm{~F}_{6} \mathrm{~N}_{2},[\mathrm{M}+\mathrm{H}]^{+}: 349.1134$, Found: 349.1128.

HPLC analysis condition:

Solvent: $n$-hexane/ iso-propanol $=95: 5$

Column: CHIRALPAK IC column (Nomura Chemical Co., Ltd.; inner diameter $4.6 \mathrm{~mm} \times$ length $250 \mathrm{~mm}$; particle size $=5 \mu \mathrm{m}$ )

Column temperature: $25^{\circ} \mathrm{C}$

Detector: UV at $350 \mathrm{~nm}$

Flow rate: $1.0 \mathrm{~mL} / \mathrm{min}$

Injection volume: $5 \mu \mathrm{L}$

$(R, R)-\mathbf{4} \mathbf{j} \mathrm{t}_{\mathrm{R}}=5.6 \mathrm{~min},(S, S)-\mathbf{4} \mathbf{j} \mathrm{t}_{\mathrm{R}}=6.0 \mathrm{~min}$ for racemic sample of $\mathbf{4} \mathbf{j}$.

ee $>99 \%$ 


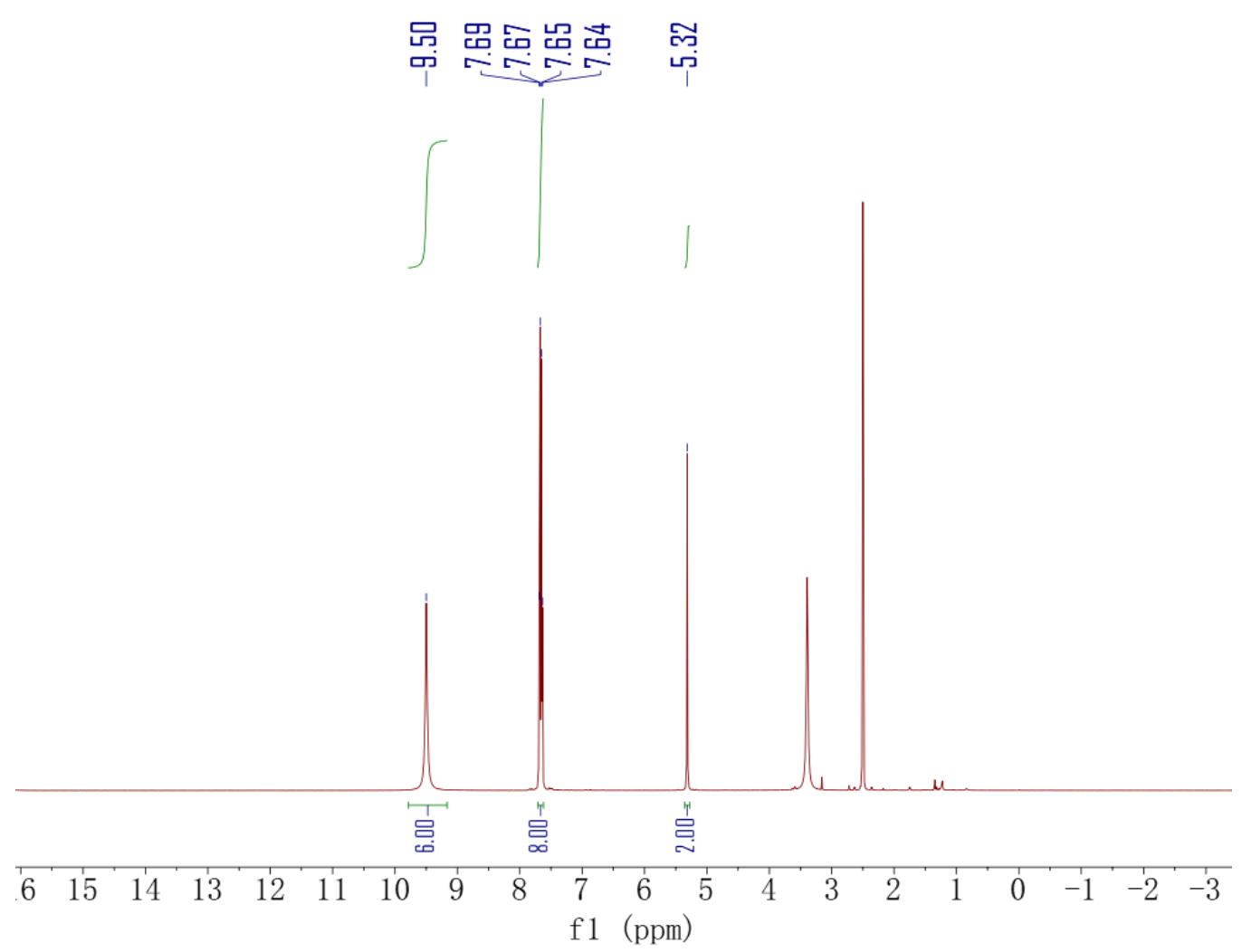

Figure S29. ${ }^{1} \mathrm{H}$ NMR of $\mathbf{4 j}$.

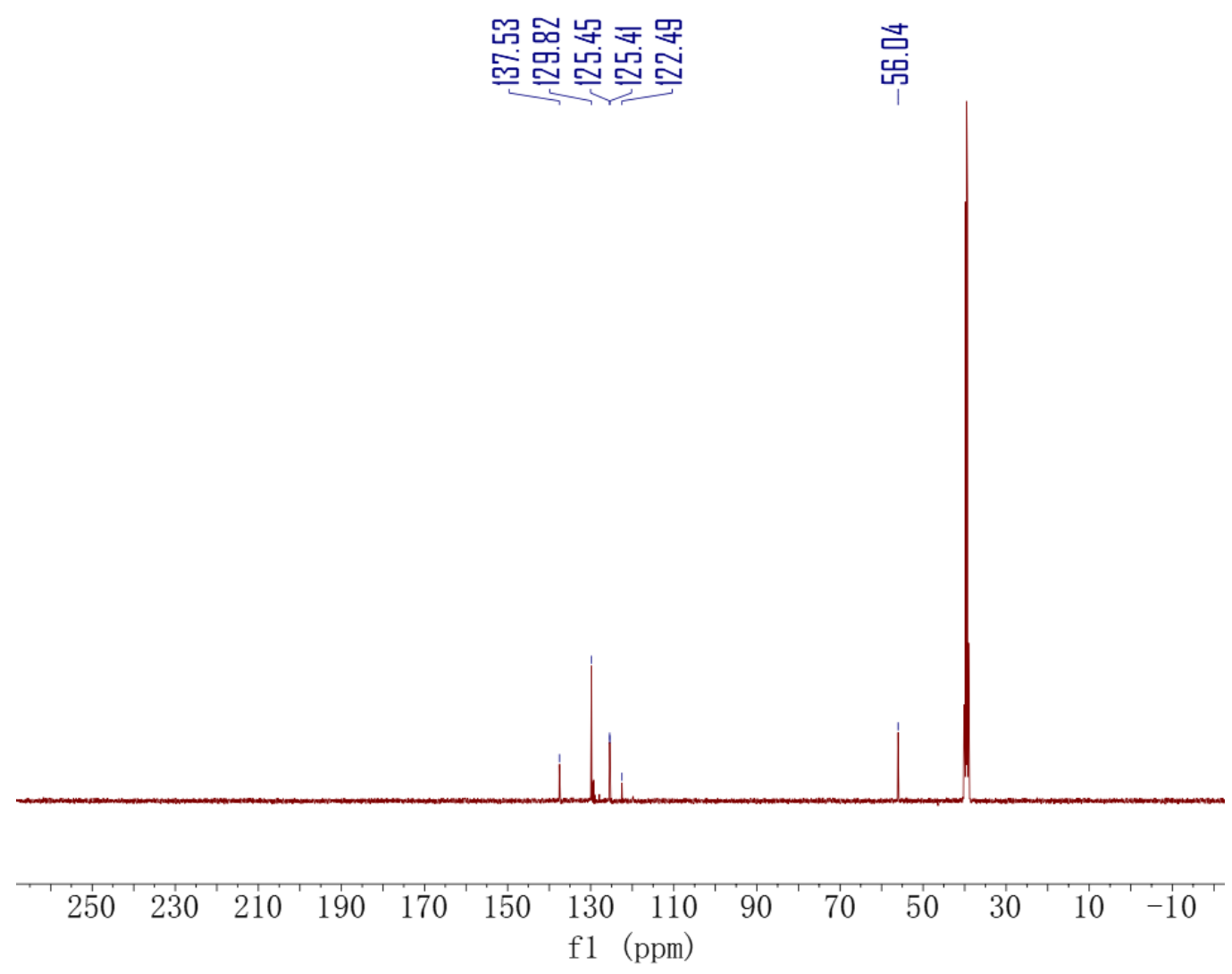

Figure S30. ${ }^{13} \mathrm{C}\left\{{ }^{1} \mathrm{H}\right\}$ NMR of $\mathbf{4 j}$. 


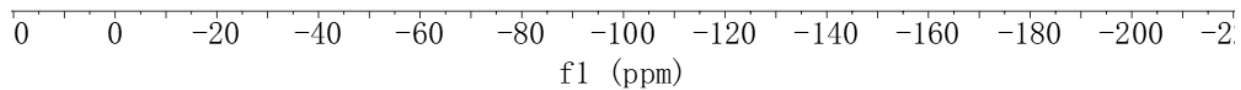

Figure S31. ${ }^{19} \mathrm{~F}\left\{{ }^{1} \mathrm{H}\right\}$ NMR of $\mathbf{4 j}$.

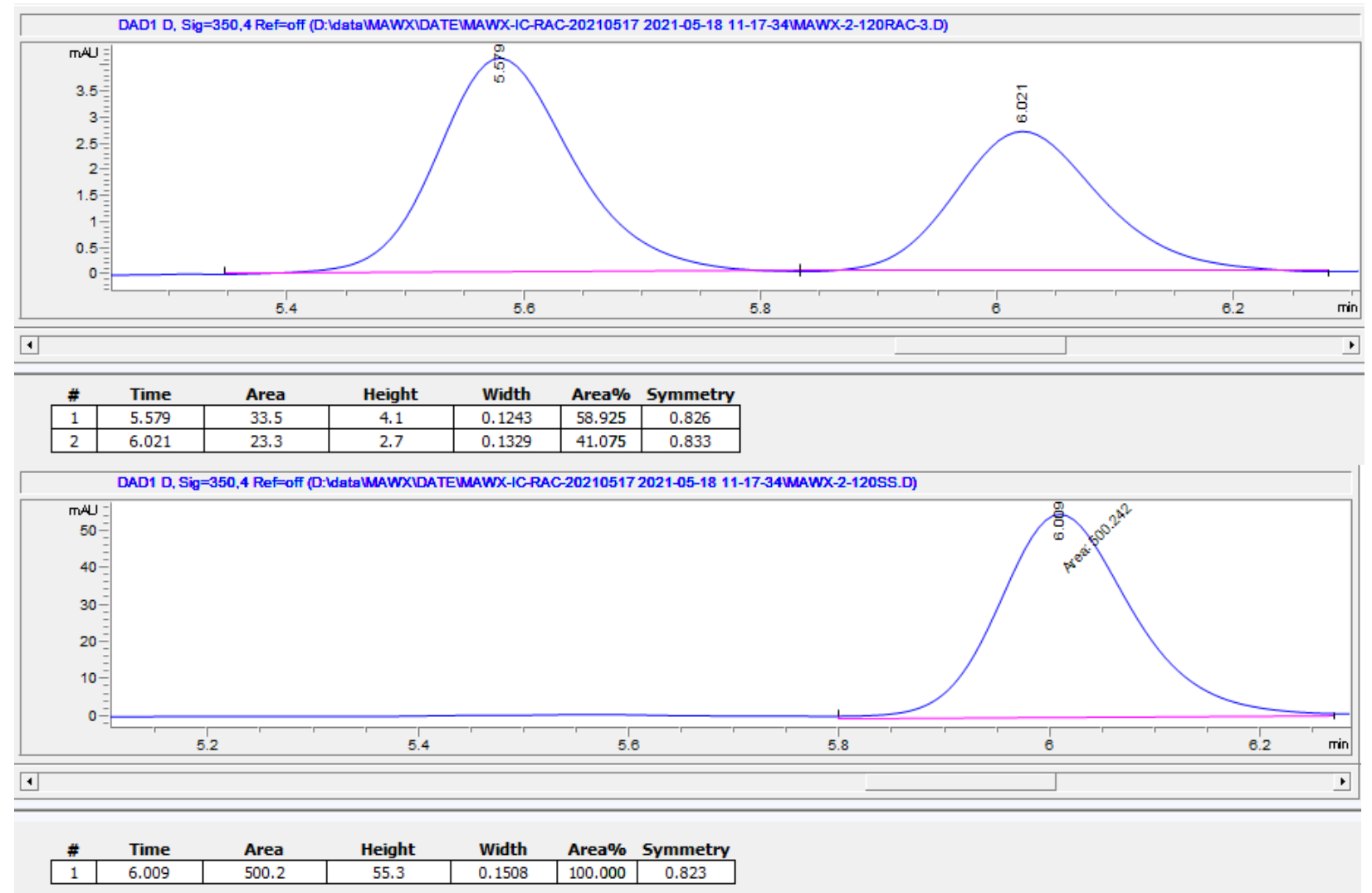

Figure S32. HPLC analysis of (top) racemic sample, and (bottom) $(S, S)$ sample of $\mathbf{4 j}$. 


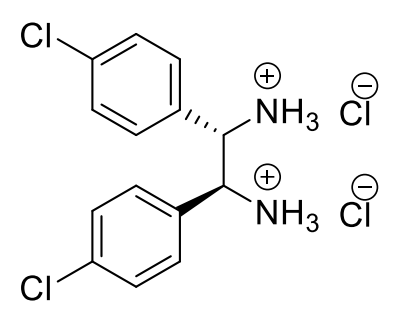

Prepared according to general procedure from $1(50 \mathrm{mg}, 0.20 \mathrm{mmol})$ and 4-chlorobenzaldehyde (2k) (60 mg, $0.43 \mathrm{mmol}$ ); washed with DCM, EA and THF to give the title compound as a white solid (51 mg, 71\%).

${ }^{1} \mathrm{H}$ NMR (DMSO-d $\left.6,500 \mathrm{MHz}, 25^{\circ} \mathrm{C}\right): \delta 9.35$ (s, $\left.6 \mathrm{H}\right), 7.42-7.36(\mathrm{~m}, 8 \mathrm{H}), 5.14(\mathrm{~s}, 2 \mathrm{H})$.

${ }^{13} \mathrm{C}\left\{{ }^{1} \mathrm{H}\right\}$ NMR (DMSO- $\left.d_{6}, 101 \mathrm{MHz}, 25{ }^{\circ} \mathrm{C}\right) \delta 133.89,132.02,130.74,128.59,55.87$.

HRMS (ESI) calculated for $\mathrm{C}_{14} \mathrm{H}_{15} \mathrm{Cl}_{2} \mathrm{~N}_{2},[\mathrm{M}+\mathrm{H}]^{+}:$: 281.0607 , Found: 281.0603.

HPLC analysis condition:

Solvent: $n$-hexane/ iso-propanol $=90: 10$

Column: CHIRALPAK IC column (Nomura Chemical Co., Ltd.; inner diameter $4.6 \mathrm{~mm} \times$ length $250 \mathrm{~mm}$; particle size $=5 \mu \mathrm{m}$ )

Column temperature: $25^{\circ} \mathrm{C}$

Detector: UV at $350 \mathrm{~nm}$

Flow rate: $0.6 \mathrm{~mL} / \mathrm{min}$

Injection volume: $5 \mu \mathrm{L}$

$(R, R)-\mathbf{4 k} \mathrm{t}_{\mathrm{R}}=10.6 \mathrm{~min},(S, S)-\mathbf{4} \mathbf{k} \mathrm{t}_{\mathrm{R}}=12.7 \mathrm{~min}$ for racemic sample of $\mathbf{4 k}$.

ee $>99 \%$ 


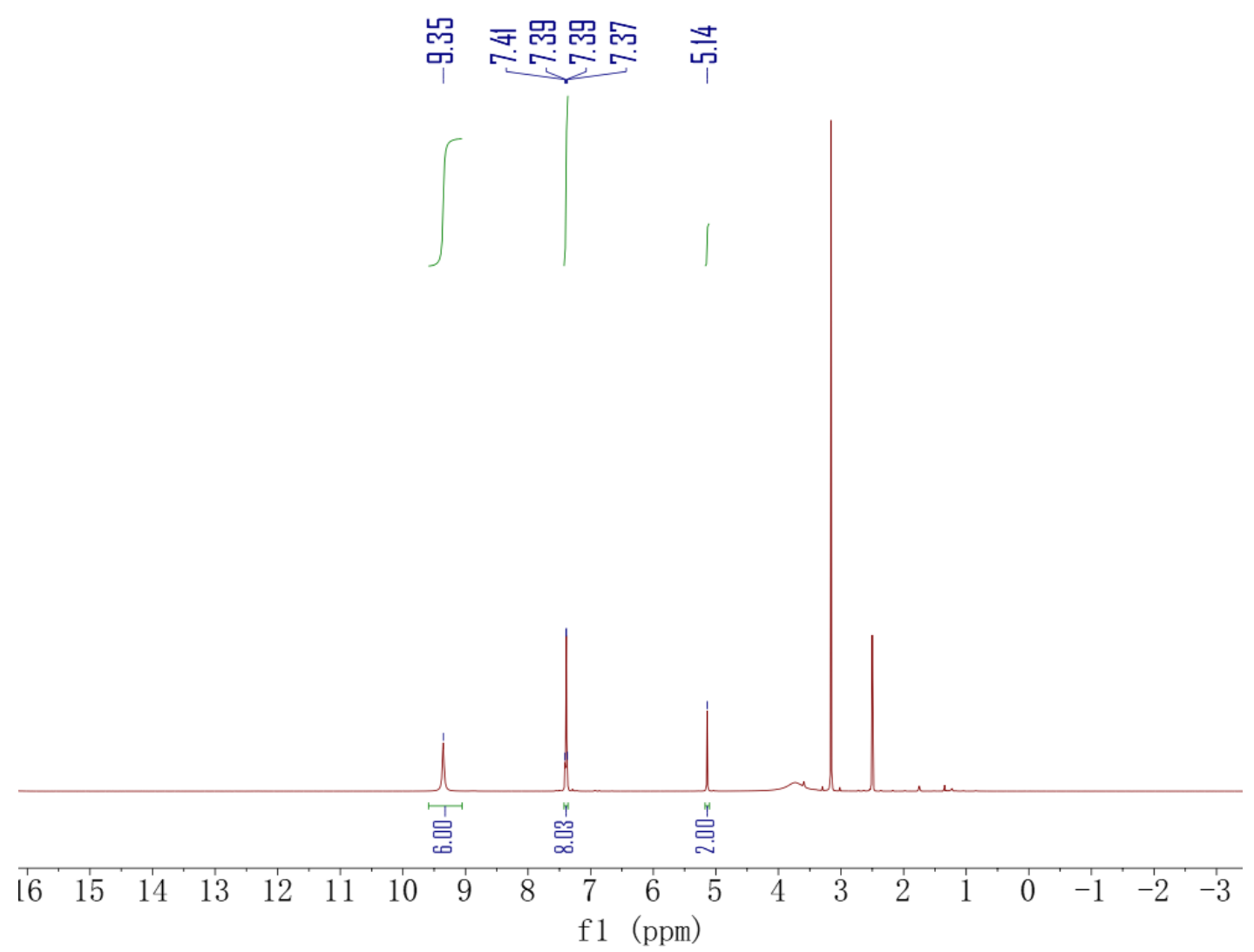

Figure S33. ${ }^{1} \mathrm{H}$ NMR of $4 \mathbf{k}$.

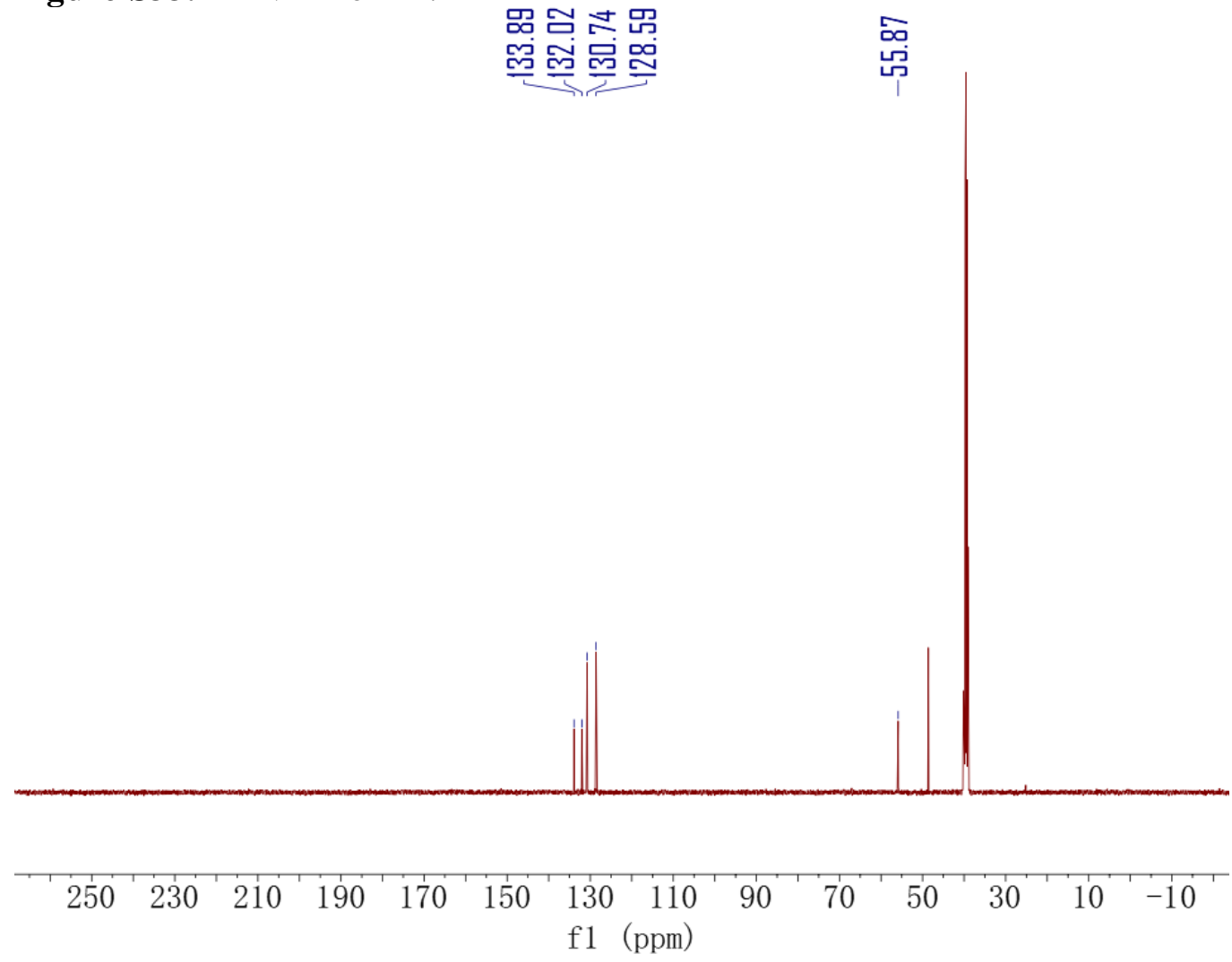

Figure S34. ${ }^{13} \mathrm{C}\left\{{ }^{1} \mathrm{H}\right\}$ NMR of $\mathbf{4 k}$. 

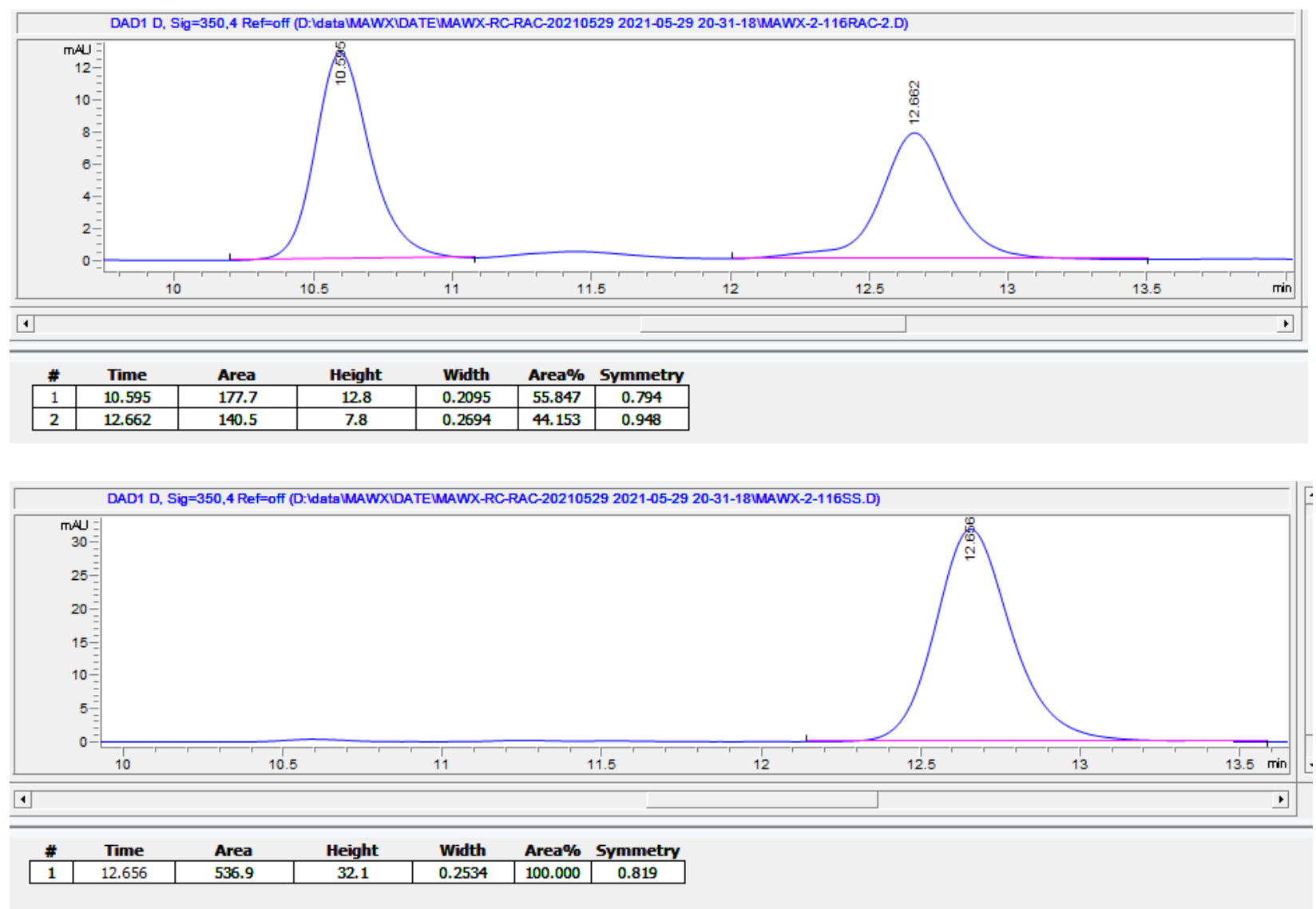

Figure S35. HPLC analysis of (top) racemic sample, and (bottom) $(S, S)$ sample of $\mathbf{4 k}$. 


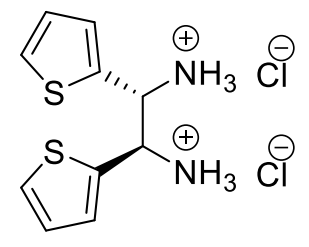

Prepared according to general procedure from 1 (50 mg, $0.20 \mathrm{mmol})$ and thiophene-2carbaldehyde (2l) (48 mg, $0.43 \mathrm{mmol}$ ); washed with DCM, EA and THF to give the title compound as a white solid (58 $\mathrm{mg}, 97 \%)$.

${ }^{1} \mathrm{H}$ NMR (DMSO- $\left.d_{6}, 500 \mathrm{MHz}, 25{ }^{\circ} \mathrm{C}\right): \delta 9.49(\mathrm{~s}, 6 \mathrm{H}), 7.51(\mathrm{dd}, J=5.0,1.2 \mathrm{~Hz}, 2 \mathrm{H}), 7.35(\mathrm{dd}$, $J=3.6,1.2 \mathrm{~Hz}, 2 \mathrm{H}), 6.98(\mathrm{dd}, J=5.1,3.6 \mathrm{~Hz}, 2 \mathrm{H}), 5.39(\mathrm{~s}, 2 \mathrm{H})$.

${ }^{13} \mathrm{C}\left\{{ }^{1} \mathrm{H}\right\}$ NMR (DMSO- $\left.d_{6}, 101 \mathrm{MHz}, 25{ }^{\circ} \mathrm{C}\right) \delta 134.39,129.31,128.01,127.11,52.50$.

HRMS (ESI) calculated for $\mathrm{C}_{10} \mathrm{H}_{13} \mathrm{~N}_{2} \mathrm{~S}_{2},[\mathrm{M}+\mathrm{H}]^{+}:$225.0515, Found: 225.0505 .

HPLC analysis condition:

Solvent: n-hexane/ iso-propanol $=99: 1$

Column: CHIRALPAK IC column (Nomura Chemical Co., Ltd.; inner diameter $4.6 \mathrm{~mm} \times$ length $250 \mathrm{~mm}$; particle size $=5 \mu \mathrm{m}$ )

Column temperature: $25{ }^{\circ} \mathrm{C}$

Detector: UV at $254 \mathrm{~nm}$

Flow rate: $0.8 \mathrm{~mL} / \mathrm{min}$

Injection volume: $5 \mu \mathrm{L}$

$(S, S)-\mathbf{4 l} \mathrm{t}_{\mathrm{R}}=45.1 \mathrm{~min},(R, R)-\mathbf{4} \mathbf{l} \mathrm{t}_{\mathrm{R}}=48.7 \mathrm{~min}$ for racemic sample of $\mathbf{4 l}$.

ee $>99 \%$ 


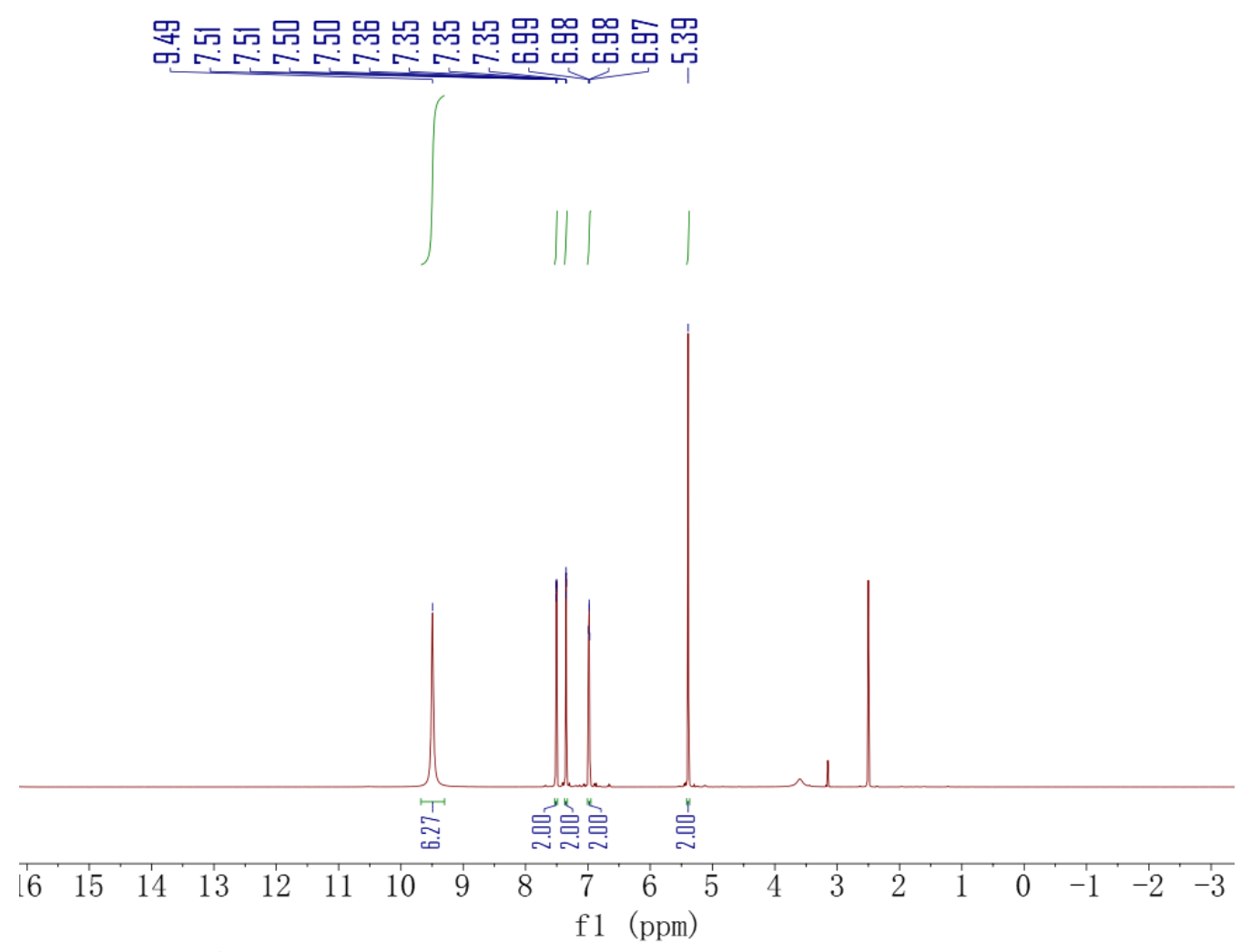

Figure S36. ${ }^{1} \mathrm{H}$ NMR of $\mathbf{4 l}$.

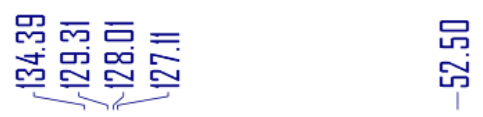

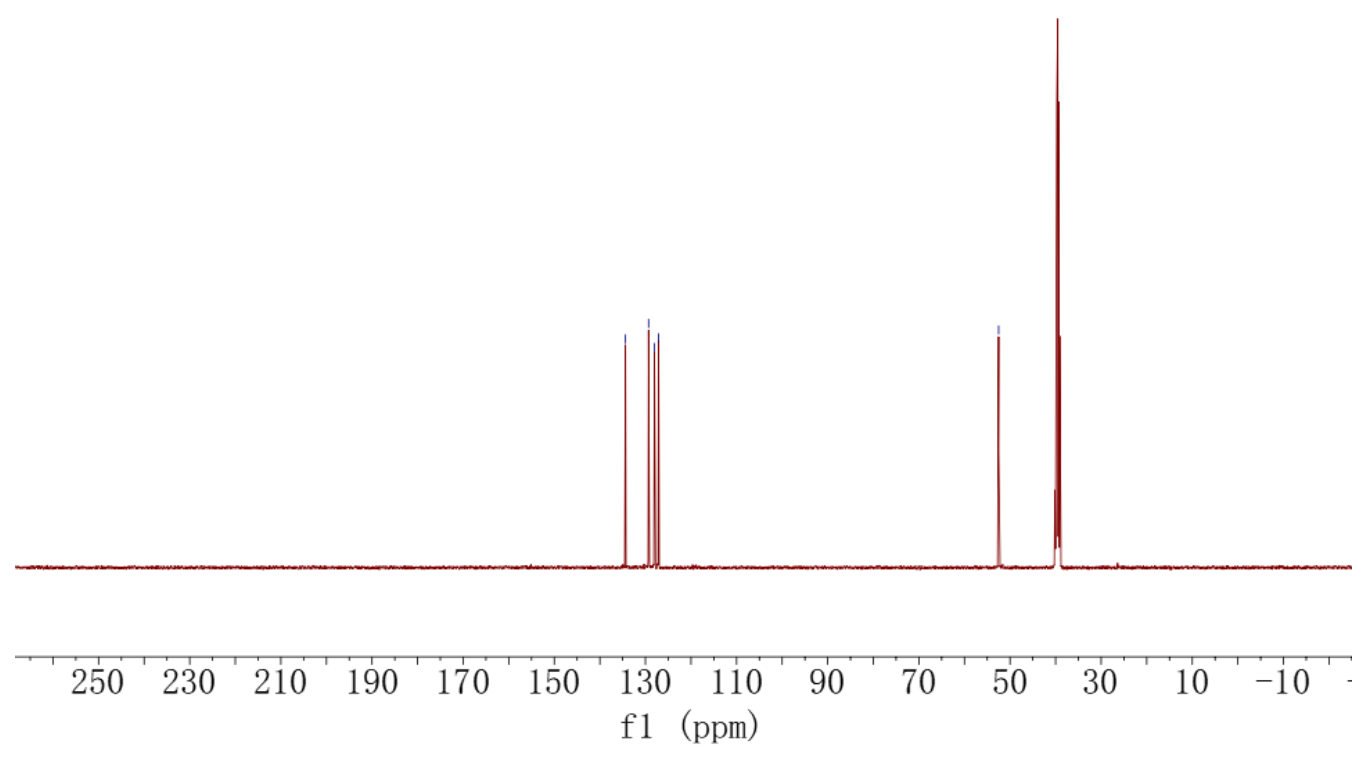

Figure S37. ${ }^{13} \mathrm{C}\left\{{ }^{1} \mathrm{H}\right\}$ NMR of 41. 

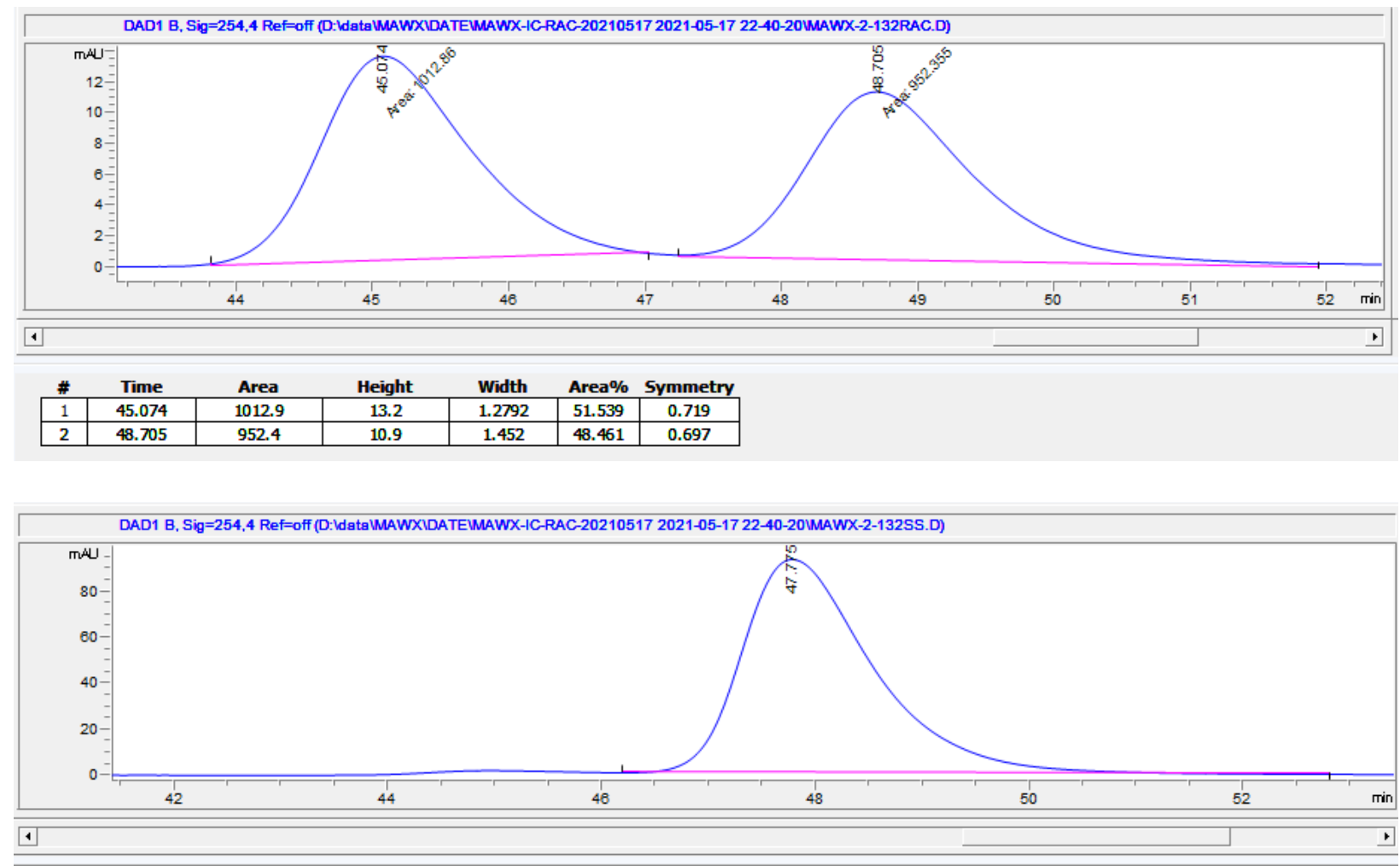

\begin{tabular}{|c|c|c|c|c|c|c|}
\hline$\#$ & Time & Area & Height & Width & Area\% & Symmetry \\
\hline 1 & 47.775 & 8027.8 & 93.9 & 1.291 & 100.000 & 0.582 \\
\hline
\end{tabular}

Figure S38. HPLC analysis of (top) racemic sample, and (bottom) $(R, R)$ sample of $\mathbf{4 l}$. 


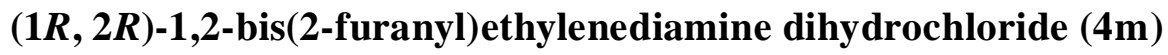

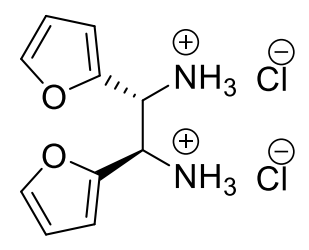

Prepared according to general procedure from $1(50 \mathrm{mg}, 0.20 \mathrm{mmol})$ and furan-2-carbaldehyde (2m) (41 mg, $0.43 \mathrm{mmol}$ ); washed with DCM, EA and THF to give the title compound as a white solid (53 mg, 99\%).

${ }^{1} \mathrm{H}$ NMR (DMSO- $\left.d_{6}, 500 \mathrm{MHz}, 25^{\circ} \mathrm{C}\right): \delta 9.45(\mathrm{~s}, 6 \mathrm{H}), 7.64(\mathrm{~d}, J=1.8 \mathrm{~Hz}, 2 \mathrm{H}), 6.55(\mathrm{~d}, J=3.3$ $\mathrm{Hz}, 2 \mathrm{H}), 6.42(\mathrm{dd}, J=3.4,1.8 \mathrm{~Hz}, 2 \mathrm{H}), 5.17(\mathrm{~s}, 2 \mathrm{H})$.

${ }^{13} \mathrm{C}\left\{{ }^{1} \mathrm{H}\right\}$ NMR (DMSO- $\left.d_{6}, 101 \mathrm{MHz}, 25{ }^{\circ} \mathrm{C}\right) \delta 145.59,144.06,111.27,110.92,49.41$.

HRMS (ESI) calculated for $\mathrm{C}_{10} \mathrm{H}_{13} \mathrm{~N}_{2} \mathrm{O}_{2},[\mathrm{M}+\mathrm{H}]^{+}:$193.0972, Found: 193.0964.

HPLC analysis condition:

Solvent: n-hexane/ iso-propanol $=99: 1$

Column: CHIRALPAK IC column (Nomura Chemical Co., Ltd.; inner diameter $4.6 \mathrm{~mm} \times$ length $250 \mathrm{~mm}$; particle size $=5 \mu \mathrm{m}$ )

Column temperature: $25{ }^{\circ} \mathrm{C}$

Detector: UV at $254 \mathrm{~nm}$

Flow rate: $0.8 \mathrm{~mL} / \mathrm{min}$

Injection volume: $5 \mu \mathrm{L}$

$(S, S)-\mathbf{4 m} \mathrm{t}_{\mathrm{R}}=39.1 \mathrm{~min},(R, R)-\mathbf{4} \mathbf{m} \mathrm{t}_{\mathrm{R}}=42.4 \mathrm{~min}$ for racemic sample of $\mathbf{4 m}$.

ee $>99 \%$ 


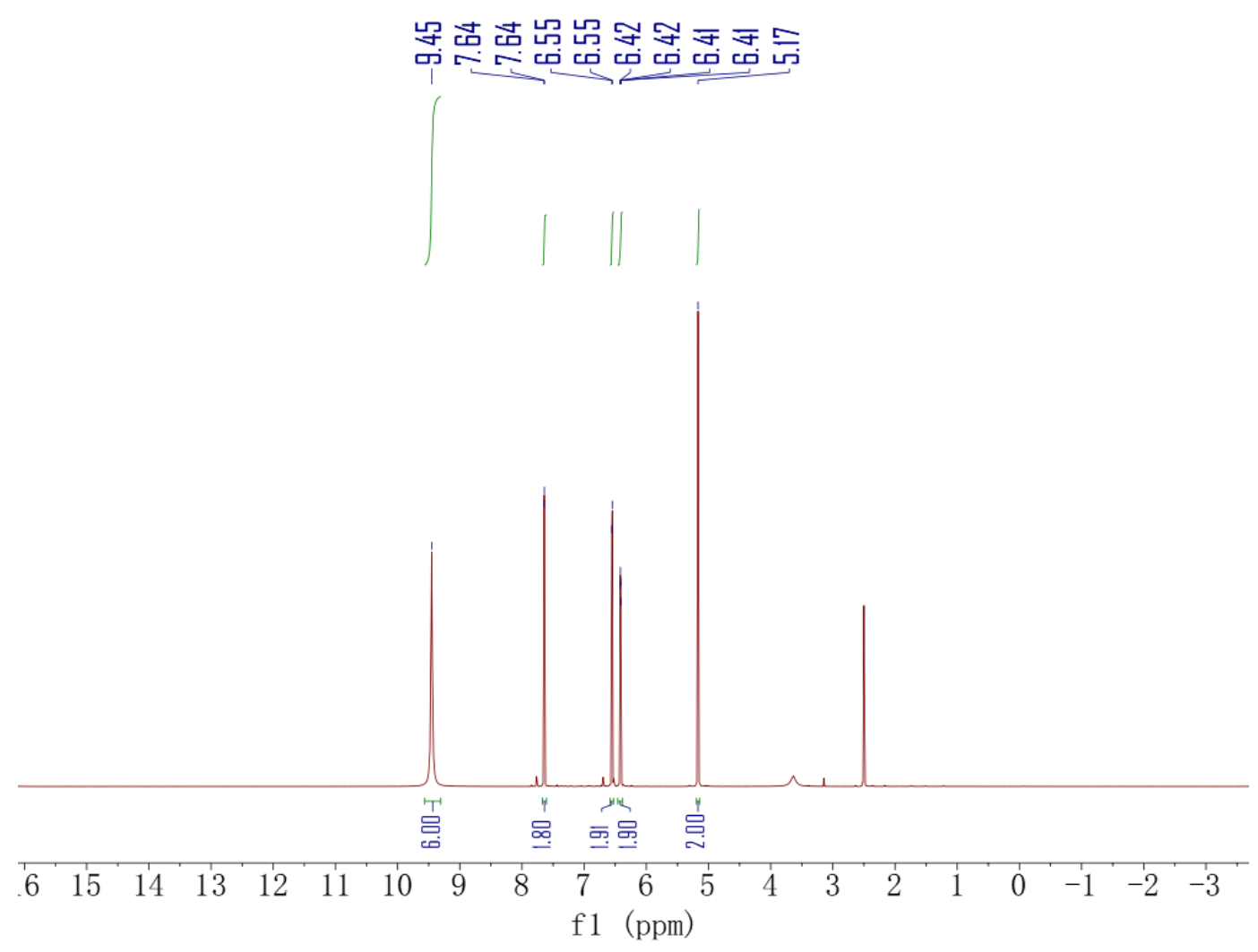

Figure S39. ${ }^{1} \mathrm{H}$ NMR of $\mathbf{4 m}$.

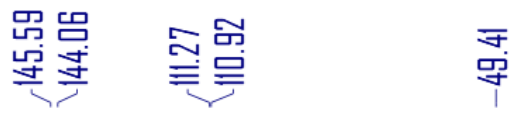

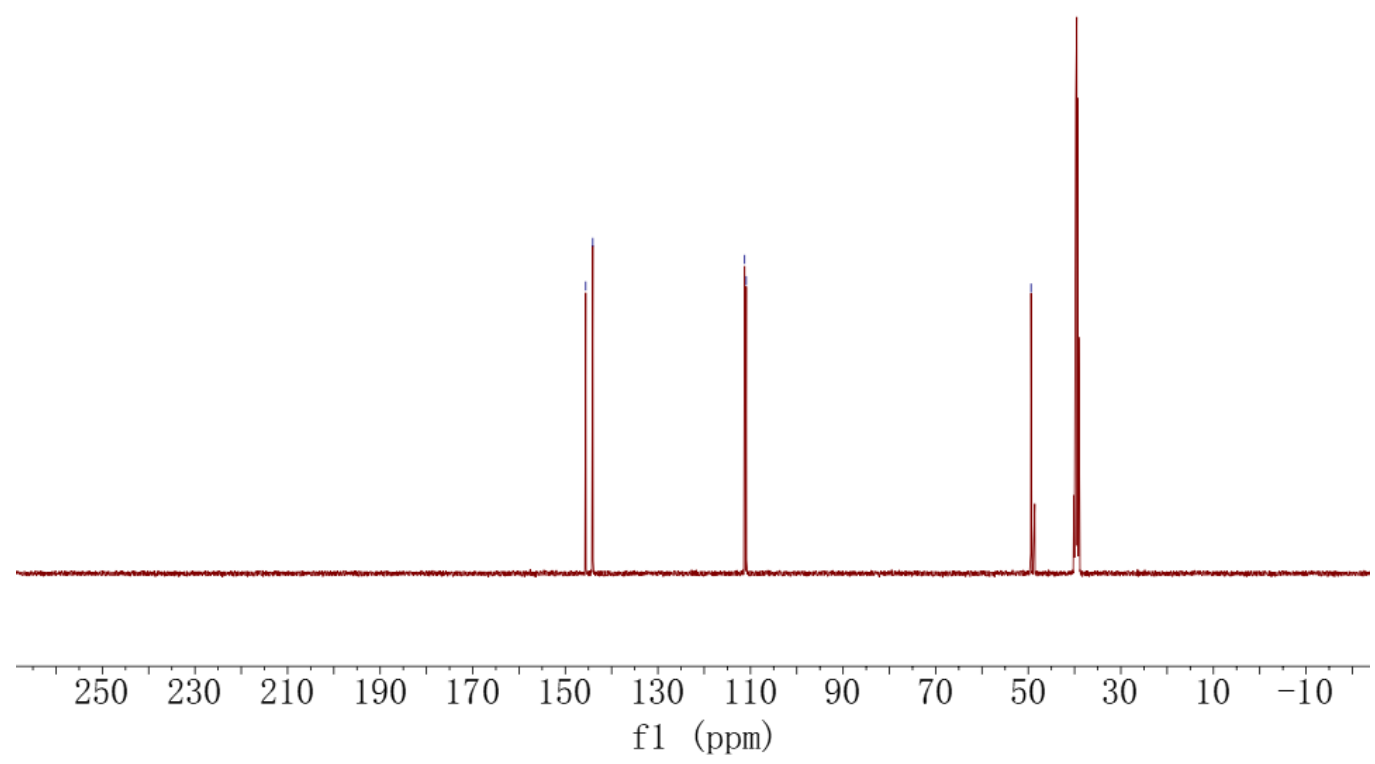

Figure S40. ${ }^{13} \mathrm{C}\left\{{ }^{1} \mathrm{H}\right\}$ NMR of $\mathbf{4 m}$. 

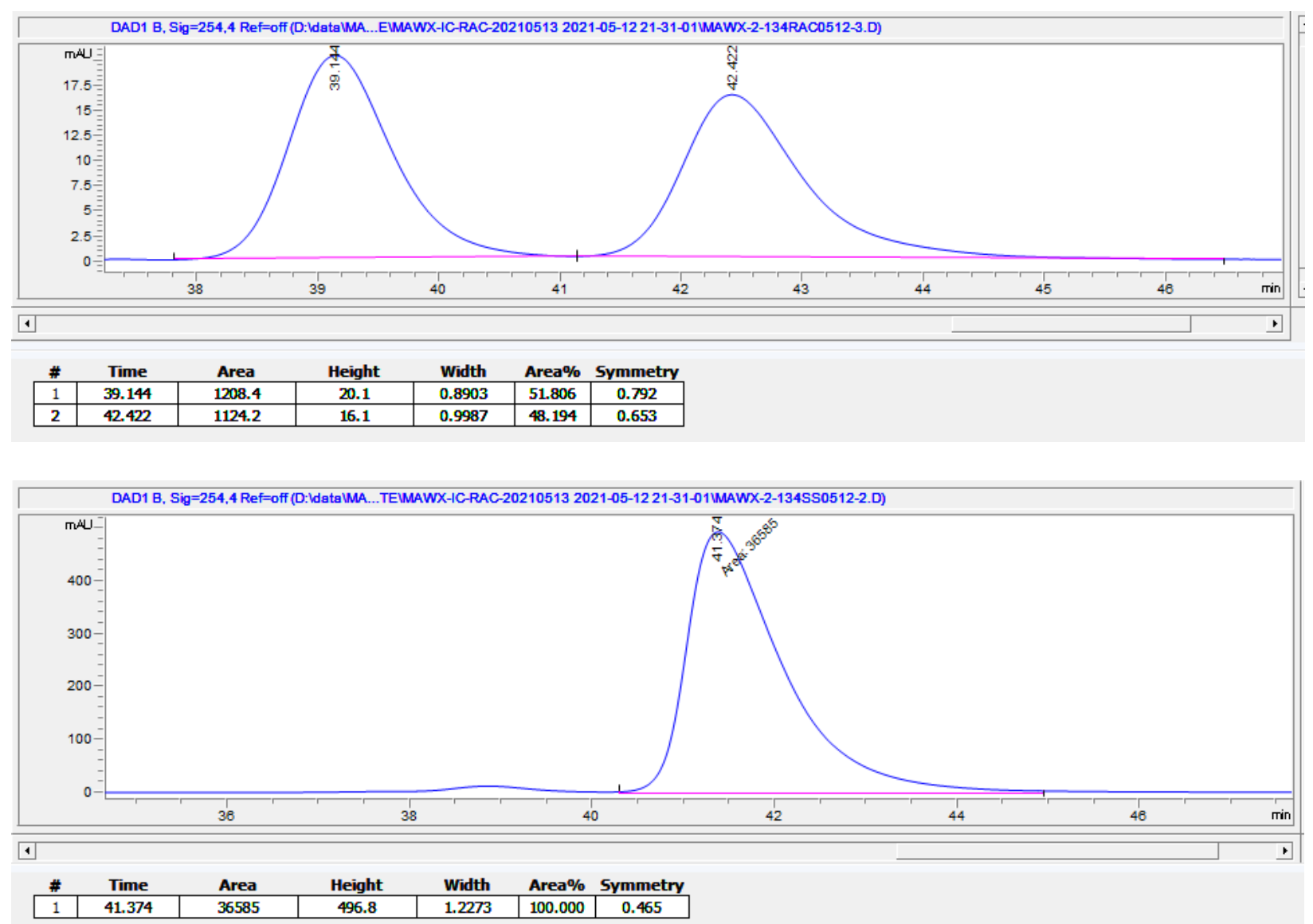

Figure S41. HPLC analysis of (top) racemic sample, and (bottom) $(R, R)$ sample of $\mathbf{4 m}$. 


\section{(2R,3R)-diethyl 2,3-diamino-2,3-dimethylsuccinate dihydrochloride (4n)}

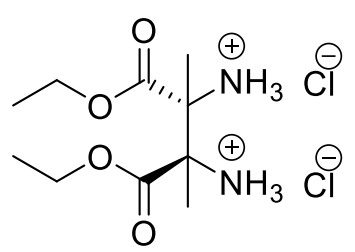

Prepared according to general procedure from $1(50 \mathrm{mg}, 0.20 \mathrm{mmol})$ and ethyl pyruvate (2n) (50 $\mathrm{mg}, 0.43 \mathrm{mmol}$ ); washed with DCM, EA and THF to give the title compound as a white solid (62 mg, $99 \%)$.

${ }^{1} \mathrm{H}$ NMR (DMSO- $\left.d_{6}, 400 \mathrm{MHz}, 25^{\circ} \mathrm{C}\right): \delta 9.37$ (s, $\left.6 \mathrm{H}\right), 4.36-4.07$ (m, $\left.4 \mathrm{H}\right), 1.66(\mathrm{~s}, 6 \mathrm{H}), 1.25$ (t, $J=7.1 \mathrm{~Hz}, 6 \mathrm{H})$.

${ }^{13} \mathrm{C}\left\{{ }^{1} \mathrm{H}\right\}$ NMR (DMSO- $\left.d_{6}, 101 \mathrm{MHz}, 25{ }^{\circ} \mathrm{C}\right) \delta 167.19,63.50,62.52,18.36,13.57$.

HRMS (ESI) calculated for $\mathrm{C}_{10} \mathrm{H}_{21} \mathrm{~N}_{2} \mathrm{O}_{4},[\mathrm{M}+\mathrm{H}]^{+}:$: 233.1496, Found: 233.1485 .

HPLC analysis condition:

Solvent: $n$-hexane/ iso-propanol $=80: 20$

Column: CHIRALPAK IC column (Nomura Chemical Co., Ltd.; inner diameter $4.6 \mathrm{~mm} \times$ length $250 \mathrm{~mm}$; particle size $=5 \mu \mathrm{m}$ )

Column temperature: $25{ }^{\circ} \mathrm{C}$

Detector: UV at $254 \mathrm{~nm}$

Flow rate: $0.8 \mathrm{~mL} / \mathrm{min}$

Injection volume: $5 \mu \mathrm{L}$

$(S, S)-\mathbf{4 n} \mathrm{t}_{\mathrm{R}}=10.3 \mathrm{~min},(R, R)-\mathbf{4 n} \mathrm{t}_{\mathrm{R}}=12.4 \mathrm{~min}$ for racemic sample of $\mathbf{4 n}$.

ee $>99 \%$ 


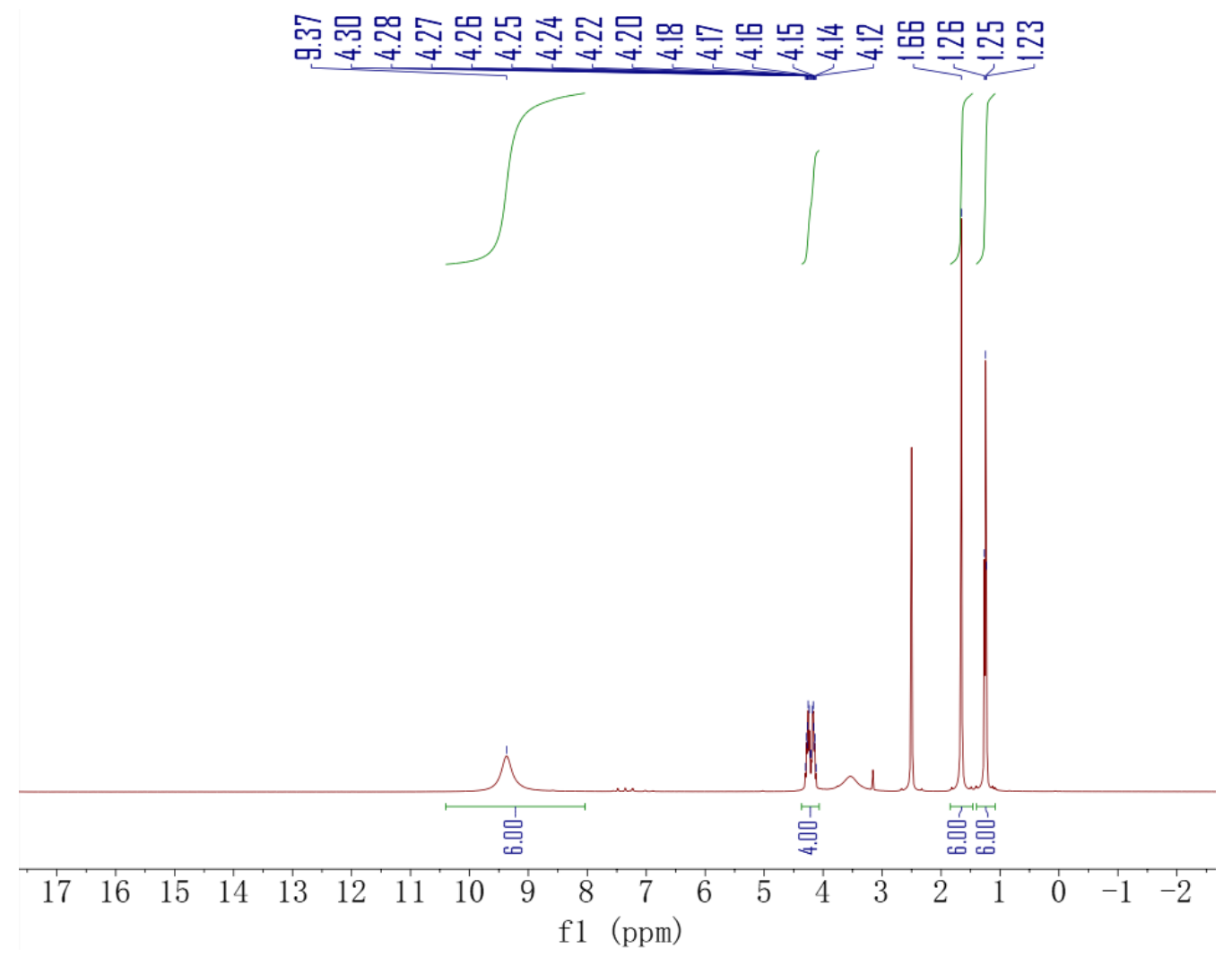

Figure S42. ${ }^{1} \mathrm{H}$ NMR of $\mathbf{4 n}$.

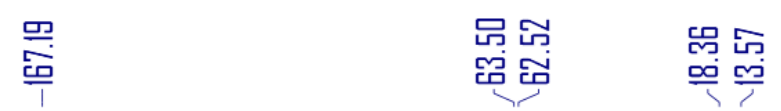

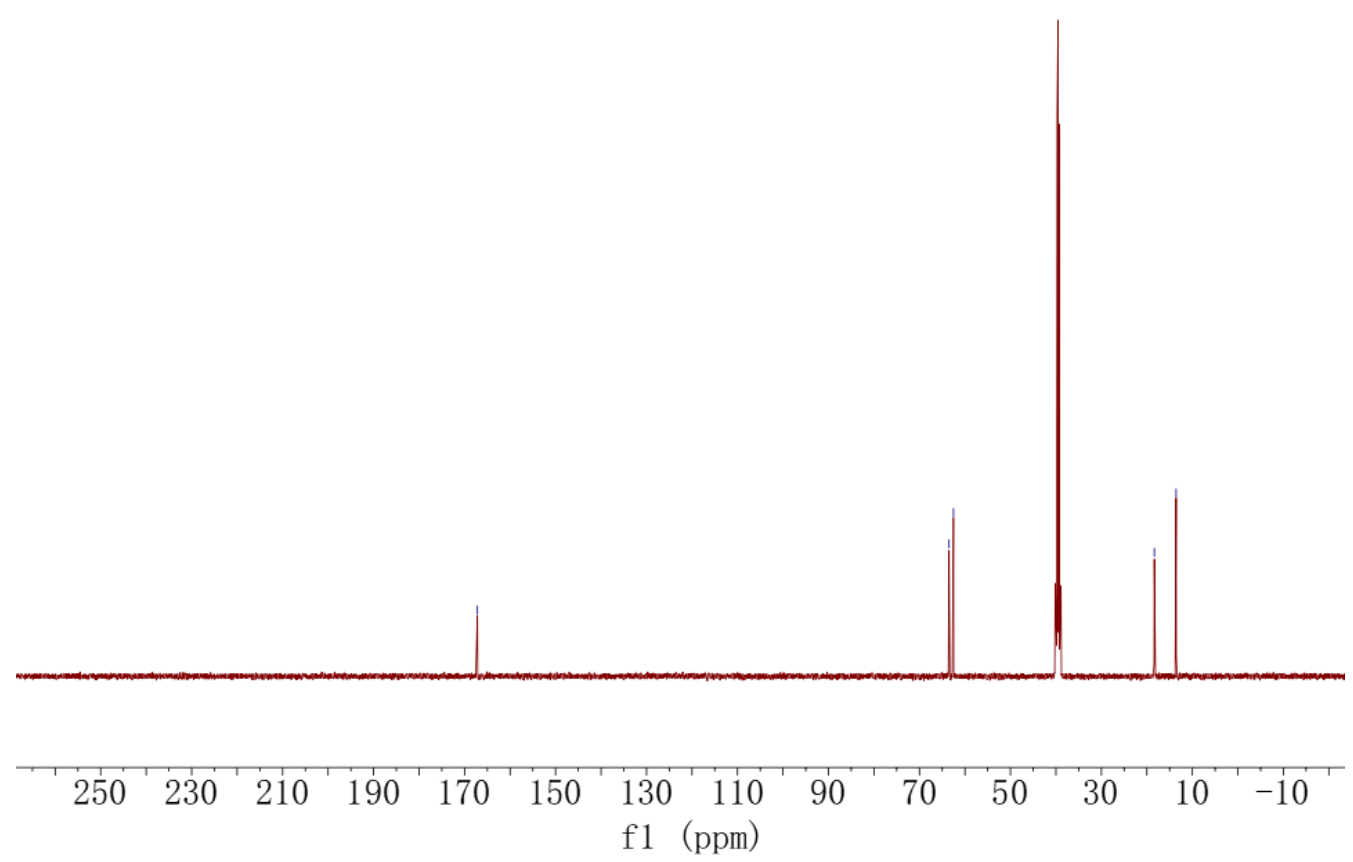

Figure S43. ${ }^{13} \mathrm{C}\left\{{ }^{1} \mathrm{H}\right\}$ NMR of $4 \mathbf{n}$. 


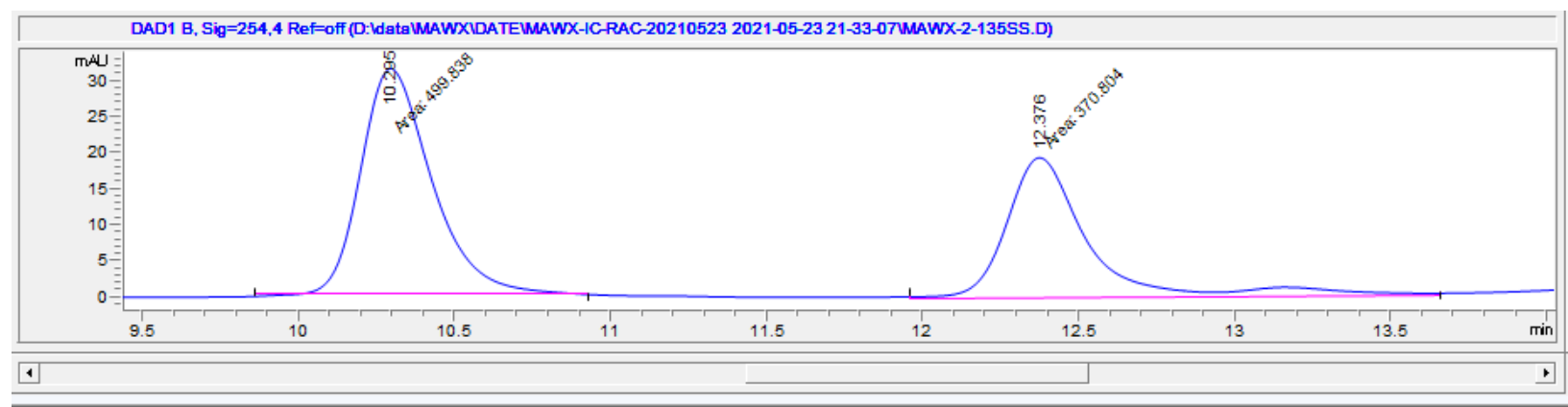

\begin{tabular}{|c|c|c|c|c|c|c|} 
\# & Time & Area & Height & Width & \multicolumn{2}{c|}{ Area\% } \\
\hline 1 & 10.295 & 499.8 & 31.3 & 0.2661 & 57.410 & 0.702 \\
\hline 2 & 12.376 & 370.8 & 19.4 & 0.3183 & 42.590 & 0.615 \\
\hline
\end{tabular}

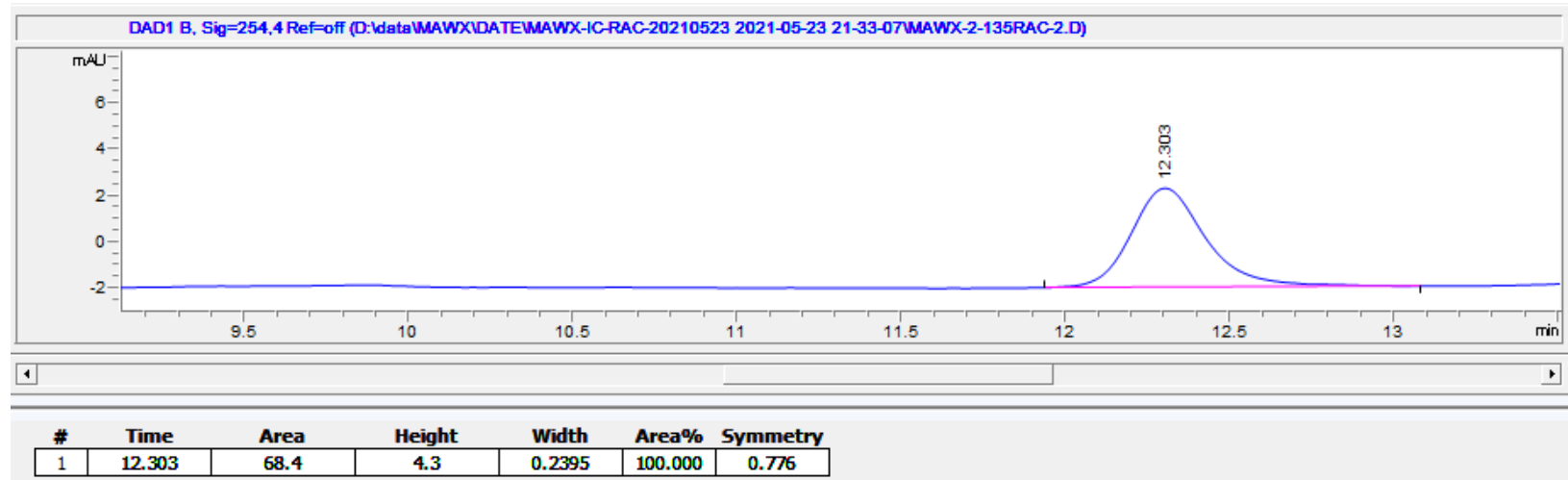

Figure S44. HPLC analysis of (top) racemic sample, and (bottom) $(R, R)$ sample of $\mathbf{4 n}$. 


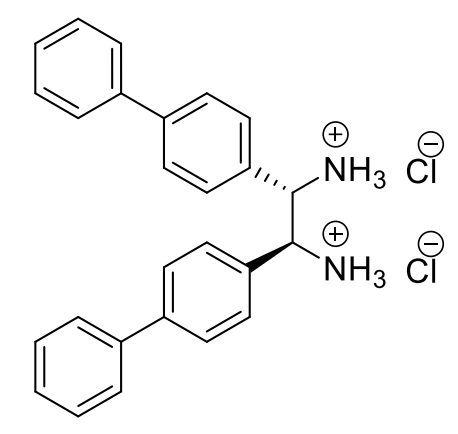

Prepared according to general procedure from 1 (50 mg, $0.20 \mathrm{mmol})$ and (1,1'-biphenyl)-4carbaldehyde (2o) (78 mg, $0.43 \mathrm{mmol})$; washed with DCM, EA and THF to give the title compound as a white solid (71 $\mathrm{mg}, 80 \%)$.

${ }^{1} \mathrm{H}$ NMR (DMSO- $\left.d_{6}, 400 \mathrm{MHz}, 25{ }^{\circ} \mathrm{C}\right): \delta 9.46(\mathrm{~s}, 6 \mathrm{H}), 7.60(\mathrm{~d}, J=7.7 \mathrm{~Hz}, 8 \mathrm{H}), 7.54(\mathrm{~d}, J=8.1$ $\mathrm{Hz}, 4 \mathrm{H}), 7.41$ (t, $J=7.5 \mathrm{~Hz}, 4 \mathrm{H}), 7.33$ (t, $J=7.3 \mathrm{~Hz}, 2 \mathrm{H}), 5.27(\mathrm{~s}, 2 \mathrm{H})$.

${ }^{13} \mathrm{C}\left\{{ }^{1} \mathrm{H}\right\}$ NMR (DMSO- $\left.d_{6}, 101 \mathrm{MHz}, 25{ }^{\circ} \mathrm{C}\right) \delta 140.48,138.97,132.38,129.57,129.02,127.87$, 126.62, 126.56, 56.40.

HRMS (ESI) calculated for $\mathrm{C}_{26} \mathrm{H}_{25} \mathrm{~N}_{2},[\mathrm{M}+\mathrm{H}]^{+}:$365.2012, Found: 365.2007.

HPLC analysis condition:

Solvent: n-hexane/ iso-propanol $=97: 3$

Column: CHIRALPAK IC column (Nomura Chemical Co., Ltd.; inner diameter $4.6 \mathrm{~mm} \times$ length $250 \mathrm{~mm}$; particle size $=5 \mu \mathrm{m}$ )

Column temperature: $25{ }^{\circ} \mathrm{C}$

Detector: UV at $350 \mathrm{~nm}$

Flow rate: $0.8 \mathrm{~mL} / \mathrm{min}$

Injection volume: $5 \mu \mathrm{L}$

$(R, R)-\mathbf{4 o} \mathrm{t}_{\mathrm{R}}=28.0 \mathrm{~min},(S, S)-\mathbf{4 o} \mathrm{t}_{\mathrm{R}}=31.5 \mathrm{~min}$ for racemic sample of $\mathbf{4 o}$.

ee $>99 \%$ 


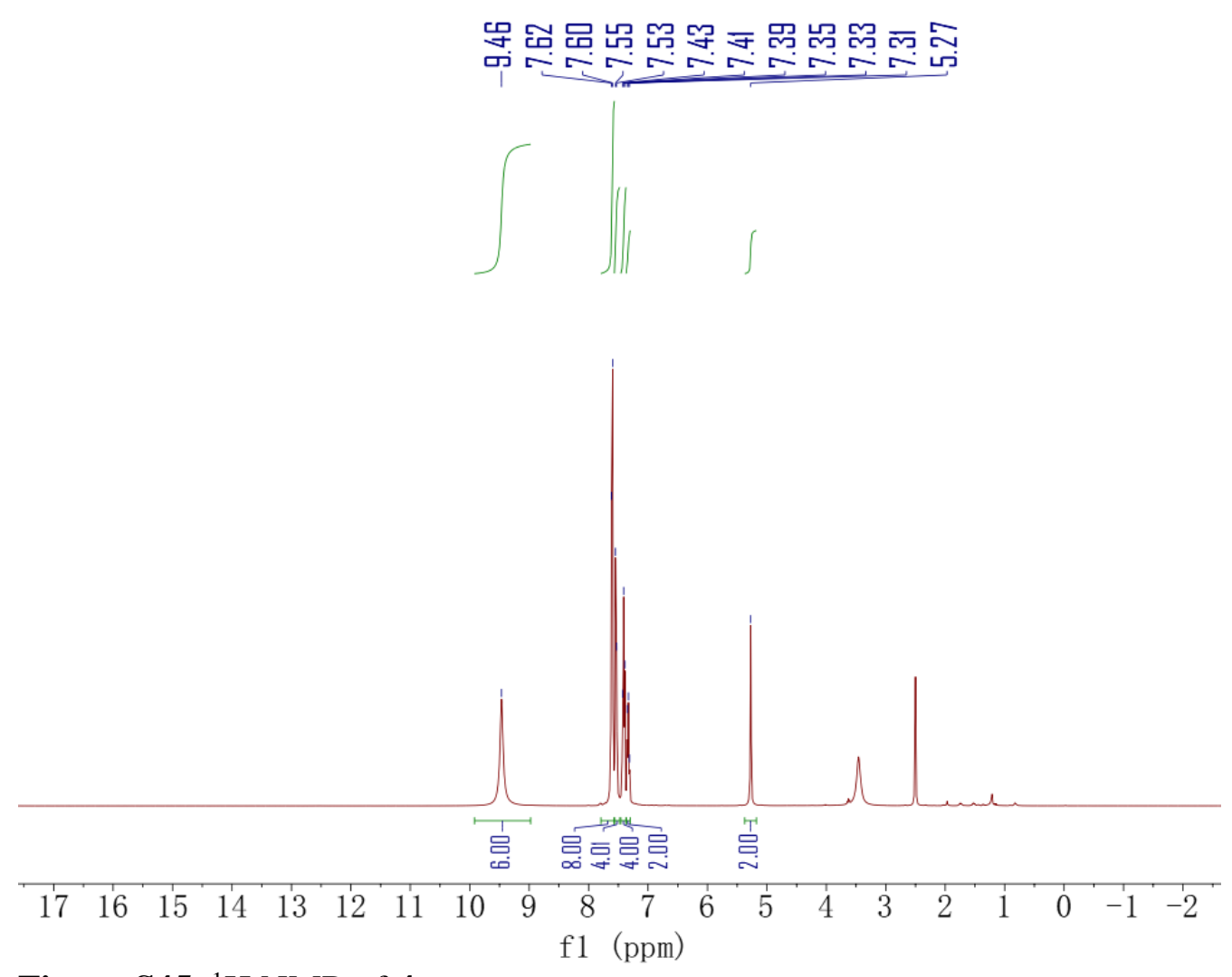

Figure S45. ${ }^{1} \mathrm{H}$ NMR of 40.

\begin{tabular}{|c|}
\hline 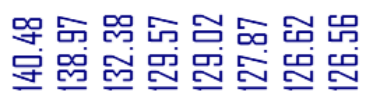 \\
\hline
\end{tabular}

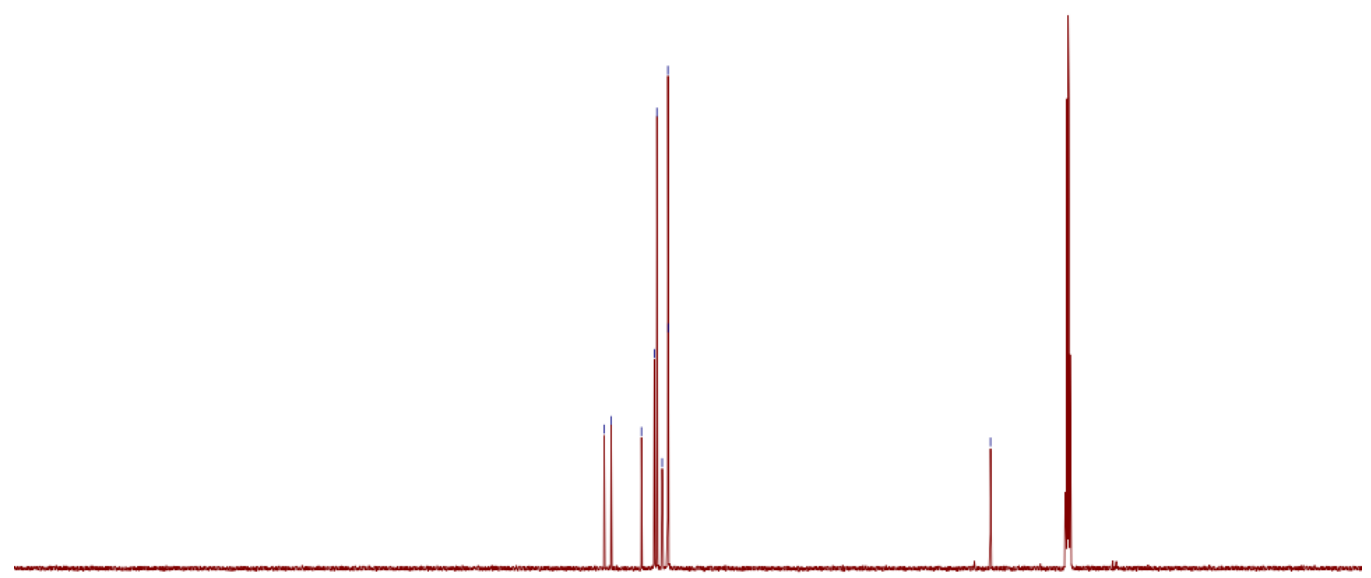

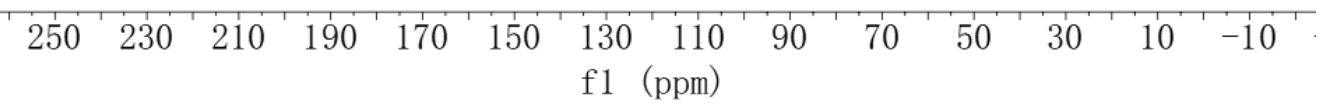

Figure S46. ${ }^{13} \mathrm{C}\left\{{ }^{1} \mathrm{H}\right\}$ NMR of 40. 

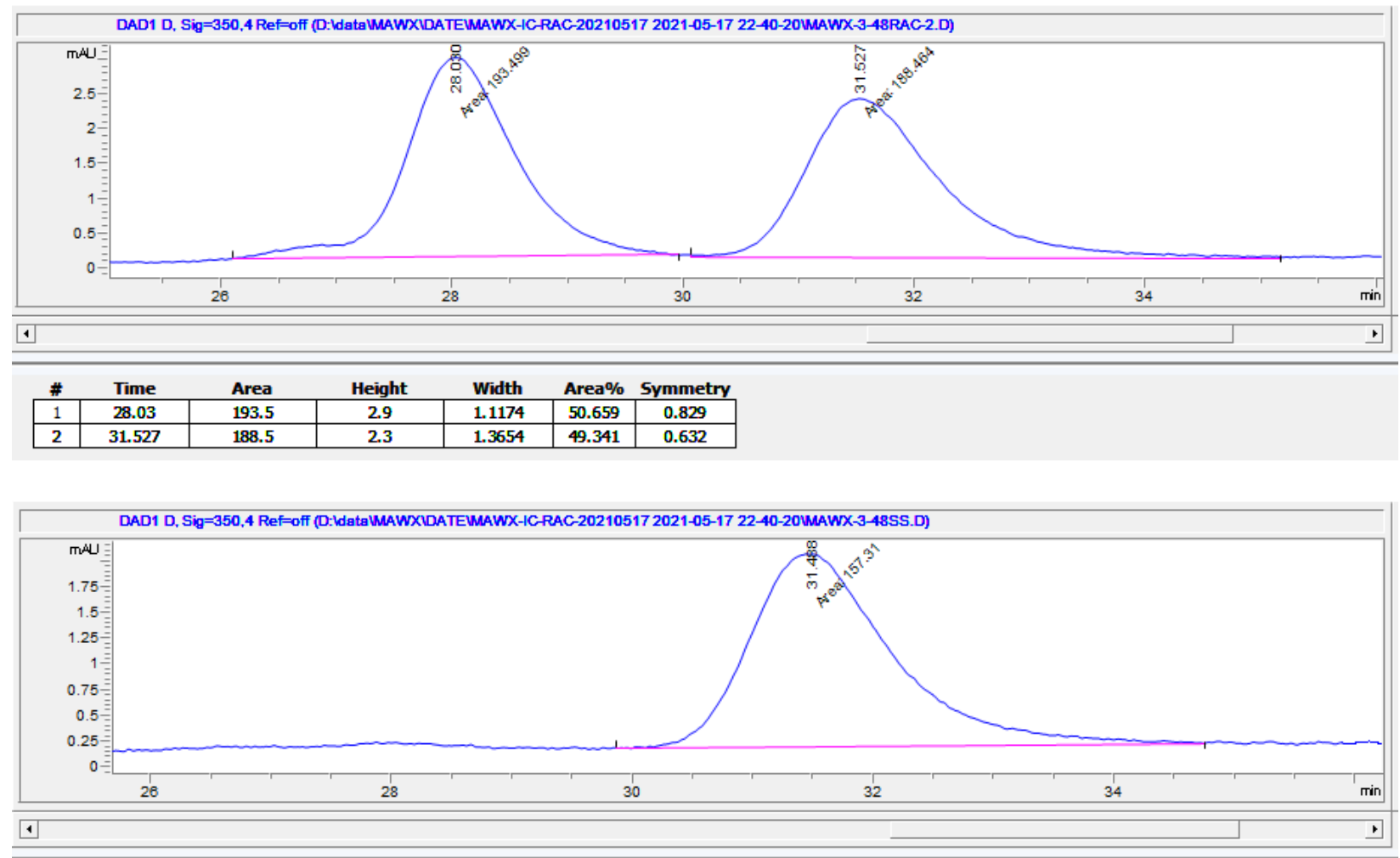

\begin{tabular}{|c|c|c|c|c|c|c|} 
\# & \multicolumn{1}{c}{ Time } & Area & \multicolumn{1}{c}{ Height } & Width & \multicolumn{2}{c|}{ Area\% } \\
\hline 1 & 31.488 & 157.3 & 1.9 & 1.3924 & 100.000 & 0.729 \\
\hline
\end{tabular}

Figure S47. HPLC analysis of (top) racemic sample, and (bottom) $(S, S)$ sample of $4 \mathbf{0 .}$ 


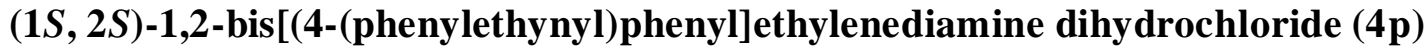

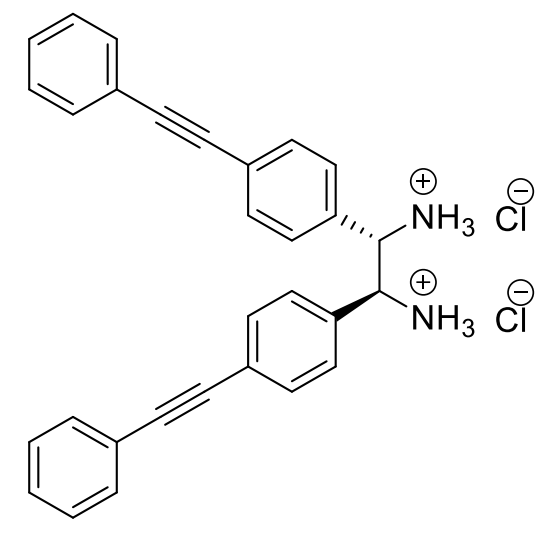

Prepared according to general procedure from $1(50 \mathrm{mg}, 0.20 \mathrm{mmol})$ and 4(phenylethynyl)benzaldehyde (2p) (89 mg, $0.43 \mathrm{mmol}$ ); washed with DCM, EA and THF to give the title compound as a white solid $(75 \mathrm{mg}, 75 \%)$.

${ }^{1} \mathrm{H}$ NMR (DMSO- $\left.d_{6}, 500 \mathrm{MHz}, 25^{\circ} \mathrm{C}\right): \delta 9.48$ (s, $\left.6 \mathrm{H}\right), 7.59-7.49$ (m, $\left.4 \mathrm{H}\right), 7.45$ (s, $\left.8 \mathrm{H}\right), 7.43-$ $7.28(\mathrm{~m}, 6 \mathrm{H}), 5.22(\mathrm{~s}, 2 \mathrm{H})$.

${ }^{13} \mathrm{C}\left\{{ }^{1} \mathrm{H}\right\}$ NMR (DMSO- $\left.d_{6}, 126 \mathrm{MHz}, 25{ }^{\circ} \mathrm{C}\right) \delta 133.73,131.44,131.38,129.27,129.06,128.81$, $122.89,121.94,90.41,88.63,56.51$.

HRMS (ESI) calculated for $\mathrm{C}_{30} \mathrm{H}_{25} \mathrm{~N}_{2},[\mathrm{M}+\mathrm{H}]^{+}:$413.2012, Found: 413.1998.

HPLC analysis condition:

Solvent: $n$-hexane/ iso-propanol $=99: 1$

Column: CHIRALPAK IC column (Nomura Chemical Co., Ltd.; inner diameter $4.6 \mathrm{~mm} \times$ length $250 \mathrm{~mm}$; particle size $=5 \mu \mathrm{m}$ )

Column temperature: $25{ }^{\circ} \mathrm{C}$

Detector: UV at $254 \mathrm{~nm}$

Flow rate: $1.0 \mathrm{~mL} / \mathrm{min}$

Injection volume: $5 \mu \mathrm{L}$

$(S, S)-\mathbf{4} \mathbf{p} t_{\mathrm{R}}=39.9 \mathrm{~min},(R, R)-\mathbf{4} \mathbf{p} \mathrm{t}_{\mathrm{R}}=47.1 \mathrm{~min}$ for racemic sample of $\mathbf{4} \mathbf{p}$.

ee $>99 \%$ 


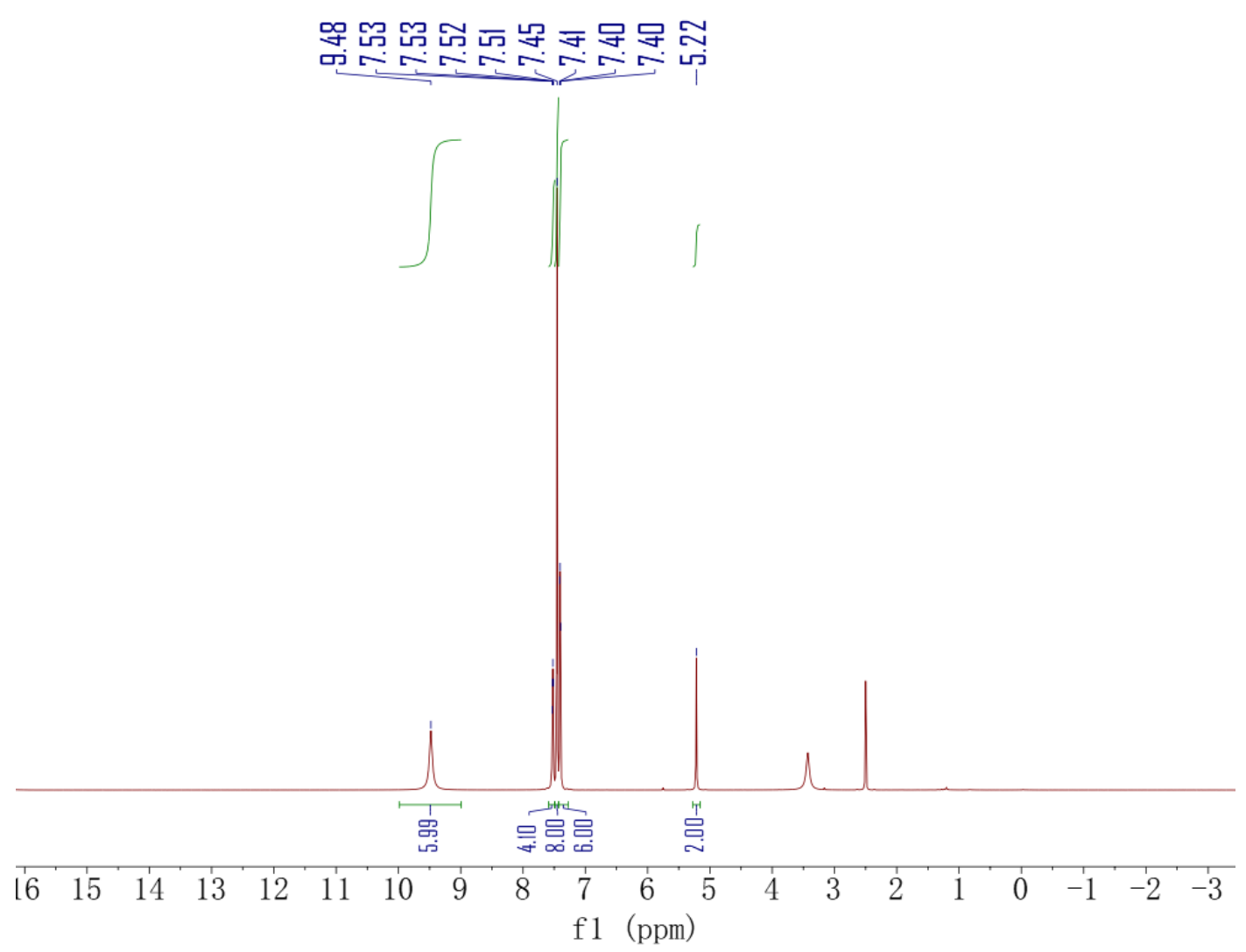

Figure S48. ${ }^{1} \mathrm{H}$ NMR of 4 p.

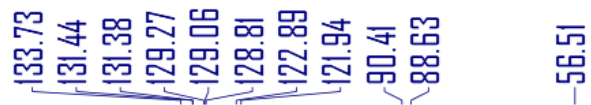

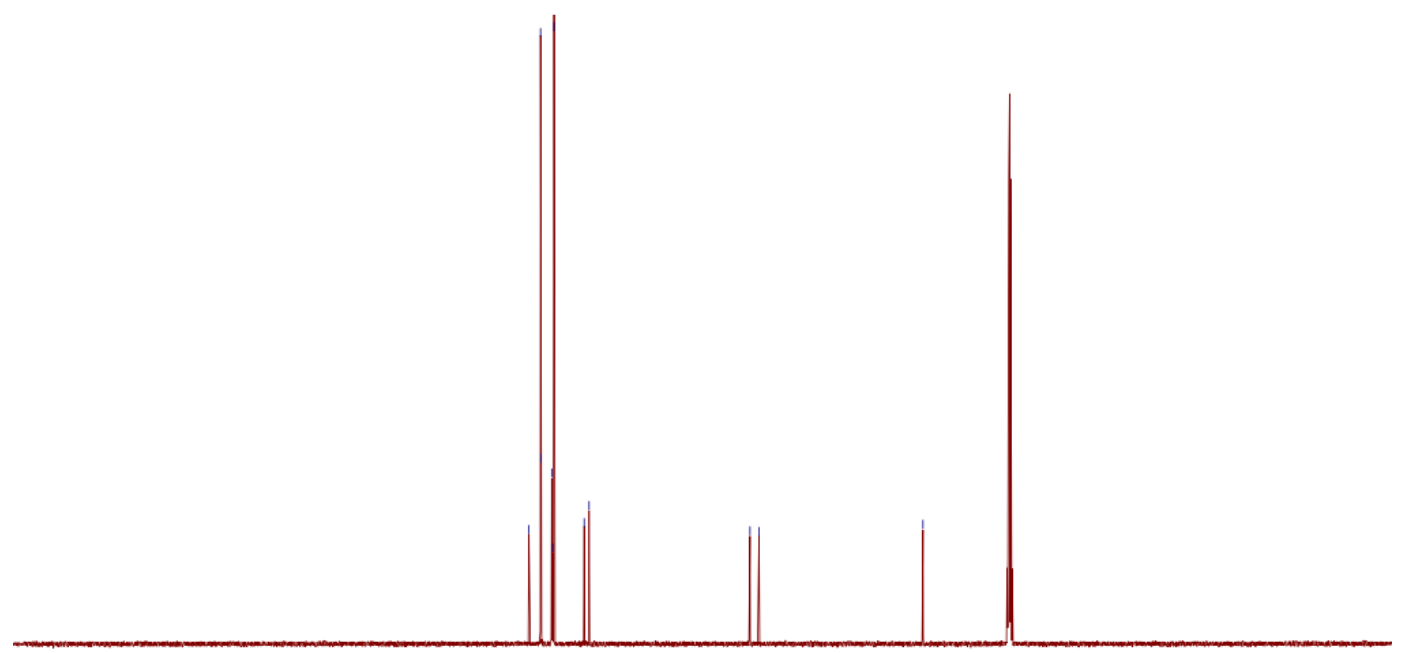

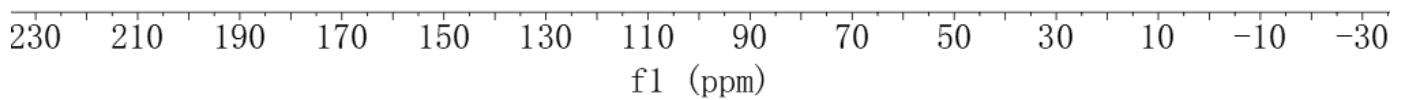

Figure S49. ${ }^{13} \mathrm{C}\left\{{ }^{1} \mathrm{H}\right\} \mathrm{NMR}$ of $\mathbf{4 p}$. 

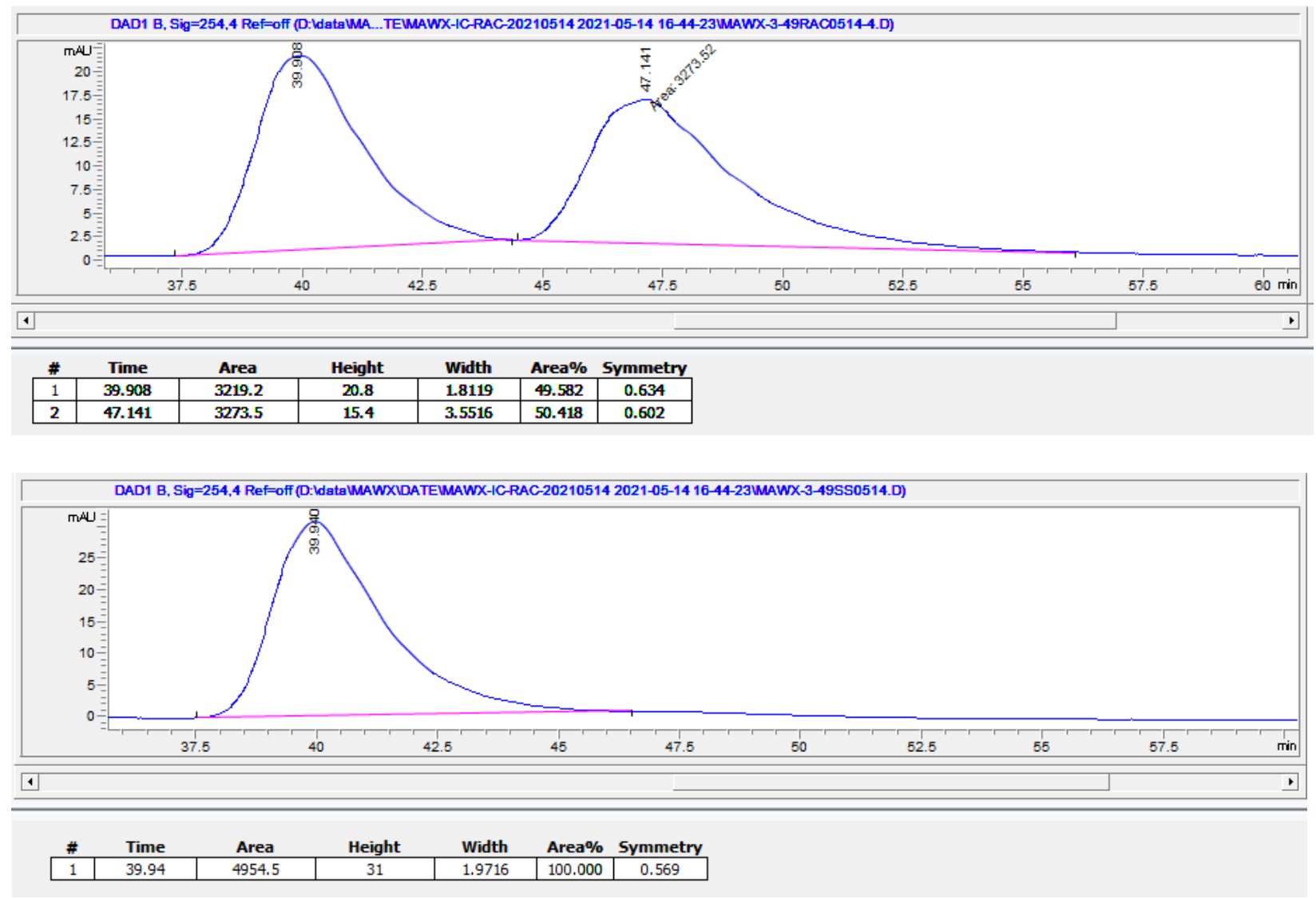

Figure S50. HPLC analysis of (top) racemic sample, and (bottom) $(S, S)$ sample of $\mathbf{4 p}$. 


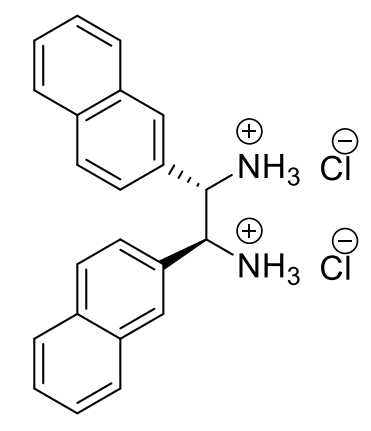

Prepared according to general procedure from 1 (50 $\mathrm{mg}, 0.20 \mathrm{mmol}$ ) and 2-naphthaldehyde (2q) (67 mg, $0.43 \mathrm{mmol}$ ); washed with DCM, EA and THF to give the title compound as a white solid (65 mg, 83\%).

${ }^{1} \mathrm{H}$ NMR (DMSO- $\left.d_{6}, 500 \mathrm{MHz}, 25^{\circ} \mathrm{C}\right): \delta 9.41(\mathrm{~s}, 6 \mathrm{H}), 7.98(\mathrm{~s}, 2 \mathrm{H}), 7.83-7.76(\mathrm{~m}, 6 \mathrm{H}), 7.53-$ $7.47(\mathrm{~m}, 6 \mathrm{H}), 5.38(\mathrm{~s}, 2 \mathrm{H})$.

${ }^{13} \mathrm{C}\left\{{ }^{1} \mathrm{H}\right\}$ NMR (DMSO- $\left.d_{6}, 101 \mathrm{MHz}, 25{ }^{\circ} \mathrm{C}\right) \delta 132.70,132.19,130.62,128.79,128.13,127.90$, $127.56,126.98,126.69,125.44,56.87$.

HRMS (ESI) calculated for $\mathrm{C}_{22} \mathrm{H}_{21} \mathrm{~N}_{2},[\mathrm{M}+\mathrm{H}]^{+}:$313.1699, Found: 313.1691.

HPLC analysis condition:

Solvent: $n$-hexane/ iso-propanol $=80: 20$

Column: CHIRALPAK IC column (Nomura Chemical Co., Ltd.; inner diameter $4.6 \mathrm{~mm} \times$ length $250 \mathrm{~mm}$; particle size $=5 \mu \mathrm{m}$ )

Column temperature: $25{ }^{\circ} \mathrm{C}$

Detector: UV at $350 \mathrm{~nm}$

Flow rate: $0.8 \mathrm{~mL} / \mathrm{min}$

Injection volume: $5 \mu \mathrm{L}$

$(R, R)-\mathbf{4 q} t_{R}=8.5 \min ,(S, S)-\mathbf{4 q} t_{R}=9.8 \mathrm{~min}$ for racemic sample of $\mathbf{4 q}$.

ee $>99 \%$ 


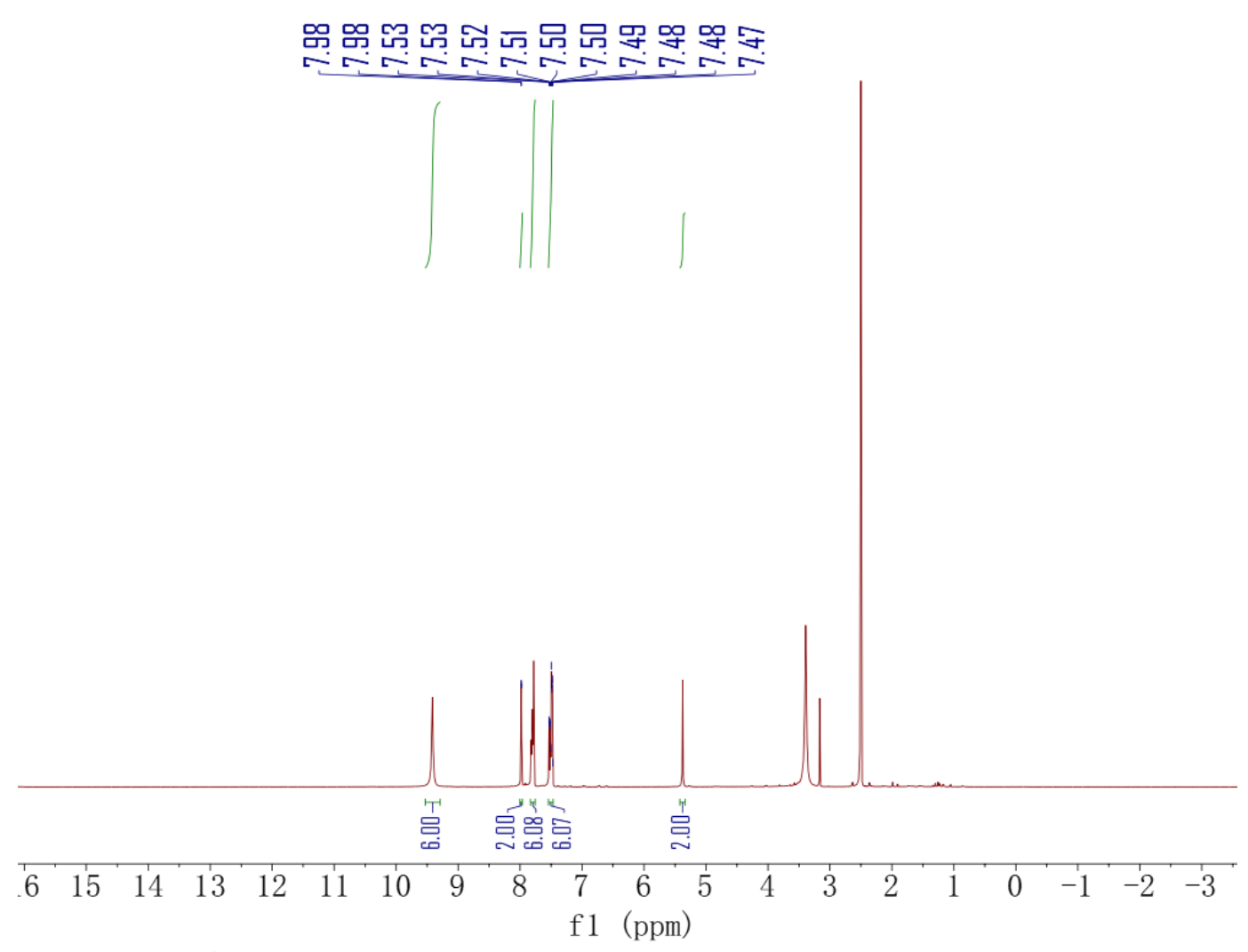

Figure S51. ${ }^{1} \mathrm{H}$ NMR of 4q.
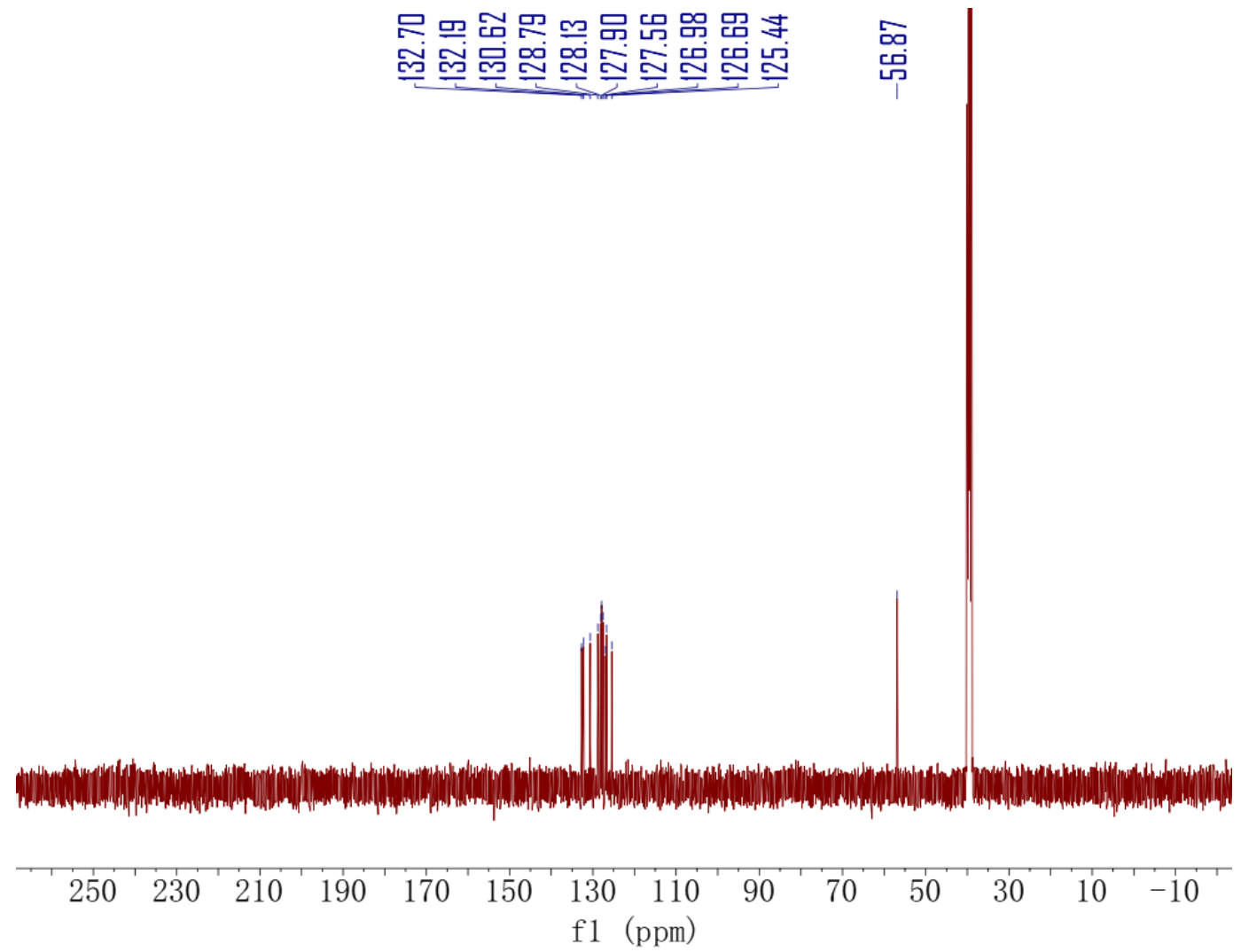

Figure S52. ${ }^{13} \mathrm{C}\left\{{ }^{1} \mathrm{H}\right\}$ NMR of $\mathbf{4 q}$. 

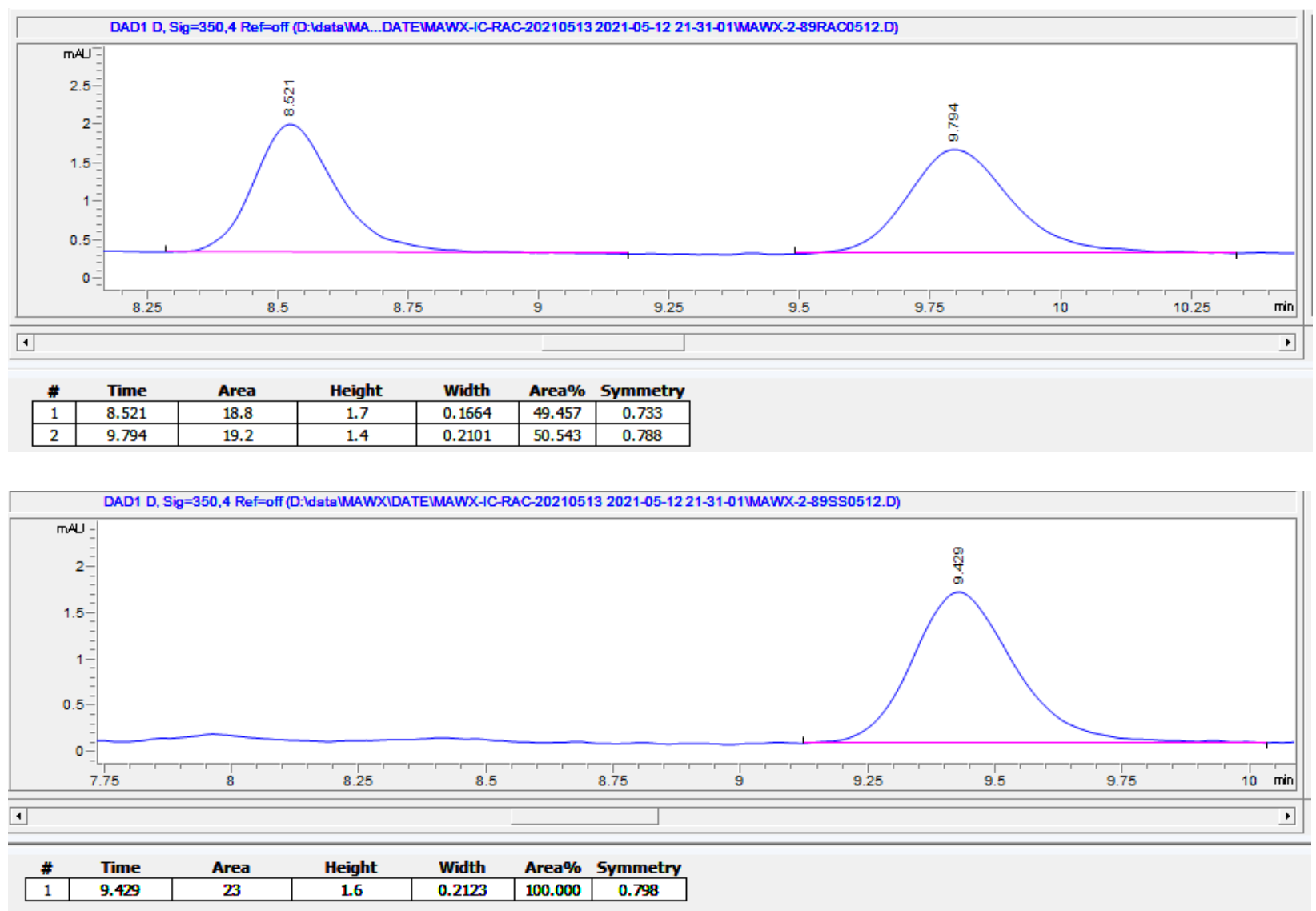

Figure S53. HPLC analysis of (top) racemic sample, and (bottom) $(S, S)$ sample of 4q. 


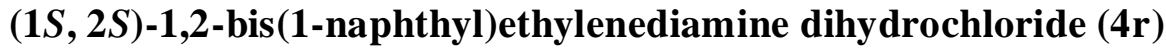

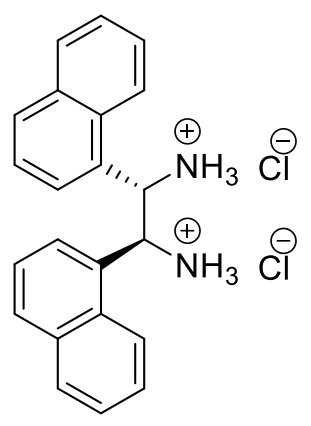

Prepared according to general procedure from $1(50 \mathrm{mg}, 0.20 \mathrm{mmol})$ and 1-naphthaldehyde (2r) (67 mg, $0.43 \mathrm{mmol}$ ); washed with DCM, EA and THF to give the title compound as a white solid (71 mg, 90\%).

${ }^{1} \mathrm{H}$ NMR (DMSO- $\left.d_{6}, 400 \mathrm{MHz}, 25^{\circ} \mathrm{C}\right): \delta 9.49(\mathrm{~s}, 6 \mathrm{H}), 8.34(\mathrm{~d}, J=8.6 \mathrm{~Hz}, 2 \mathrm{H}), 8.14(\mathrm{~d}, J=7.6$ $\mathrm{Hz}, 2 \mathrm{H}), 7.71(\mathrm{~d}, J=8.2 \mathrm{~Hz}, 2 \mathrm{H}), 7.59(\mathrm{~d}, J=8.1 \mathrm{~Hz}, 4 \mathrm{H}), 7.43(\mathrm{t}, J=7.5 \mathrm{~Hz}, 2 \mathrm{H}), 7.16(\mathrm{t}, J$ $=7.8 \mathrm{~Hz}, 2 \mathrm{H}), 6.40(\mathrm{~s}, 2 \mathrm{H})$.

${ }^{13} \mathrm{C}\left\{{ }^{1} \mathrm{H}\right\}$ NMR (DMSO- $\left.d_{6}, 101 \mathrm{MHz}, 25{ }^{\circ} \mathrm{C}\right) \delta 132.70,130.45,129.32,128.53,126.64,126.18$, $125.86,125.85,124.71,123.15,51.67$.

HRMS (ESI) calculated for $\mathrm{C}_{22} \mathrm{H}_{21} \mathrm{~N}_{2},[\mathrm{M}+\mathrm{H}]^{+}:$313.1699, Found: 313.1687.

HPLC analysis condition:

Solvent: $n$-hexane/ iso-propanol $=90: 10$

Column: CHIRALPAK IC column (Nomura Chemical Co., Ltd.; inner diameter $4.6 \mathrm{~mm} \times$ length $250 \mathrm{~mm}$; particle size $=5 \mu \mathrm{m}$ )

Column temperature: $25^{\circ} \mathrm{C}$

Detector: UV at $254 \mathrm{~nm}$

Flow rate: $0.8 \mathrm{~mL} / \mathrm{min}$

Injection volume: $5 \mu \mathrm{L}$

$(R, R)-\mathbf{4 r} \mathrm{t}_{\mathrm{R}}=10.5 \mathrm{~min},(S, S)-\mathbf{4} \mathbf{r} \mathrm{t}_{\mathrm{R}}=14.0 \mathrm{~min}$ for racemic sample of $\mathbf{4 r}$.

ee $>99 \%$ 


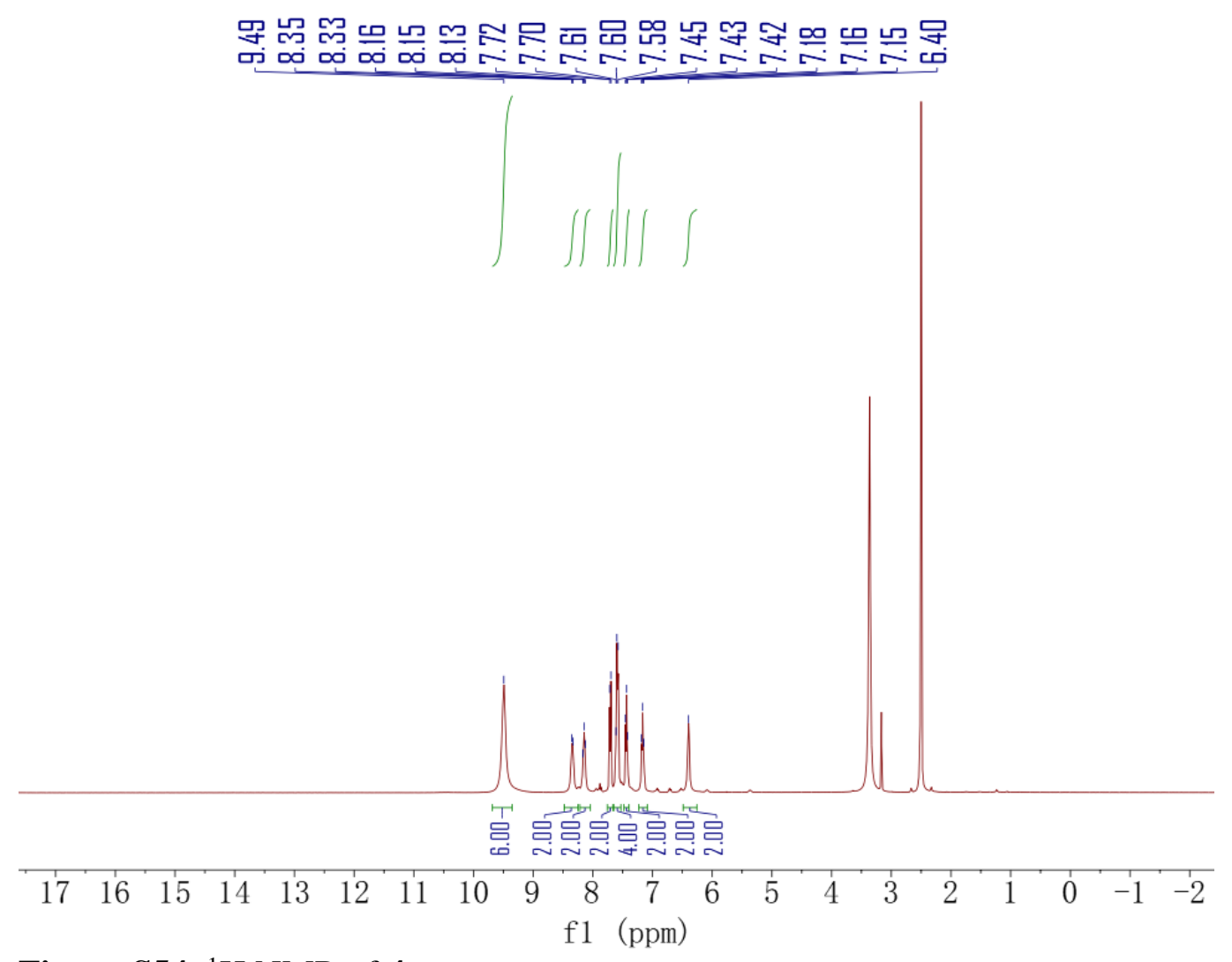

Figure S54. ${ }^{1} \mathrm{H}$ NMR of $4 r$.
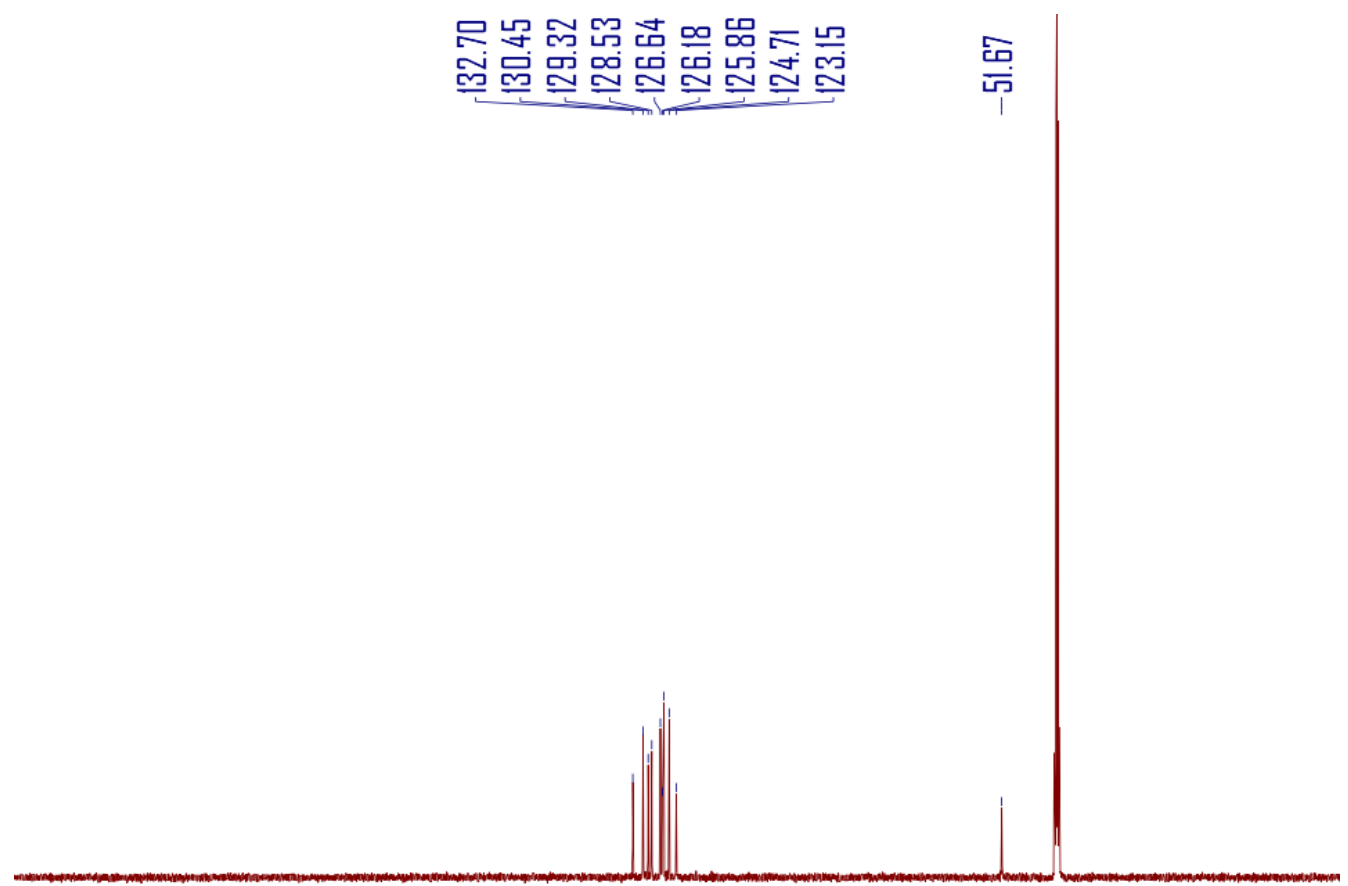

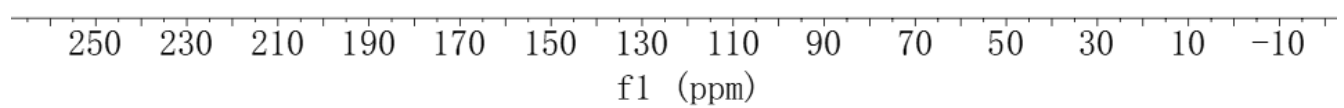

Figure S55. ${ }^{13} \mathrm{C}\left\{{ }^{1} \mathrm{H}\right\}$ NMR of $\mathbf{4 r}$. 

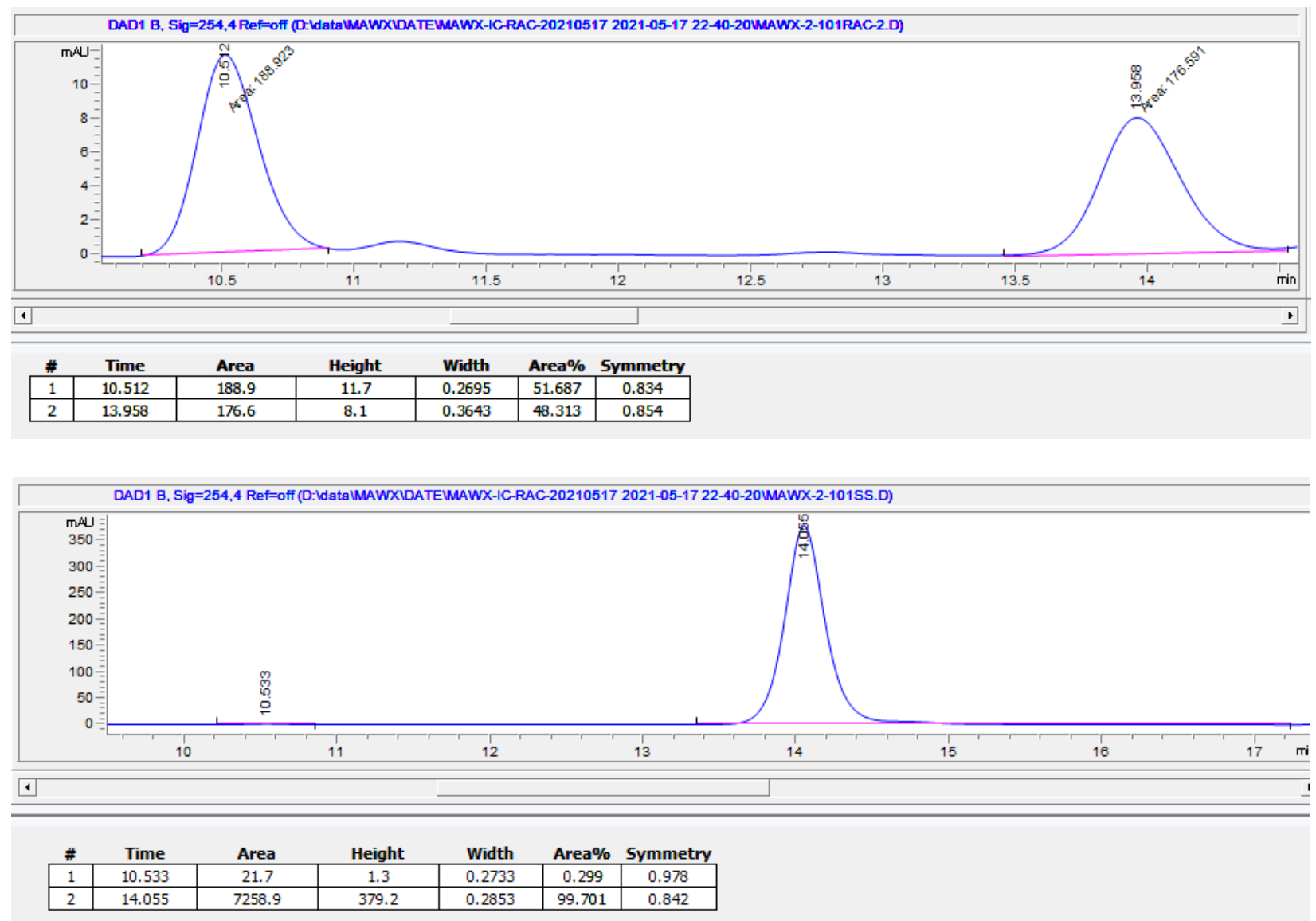

Figure S56. HPLC analysis of (top) racemic sample, and (bottom) $(S, S)$ sample of $4 \mathbf{r}$. 


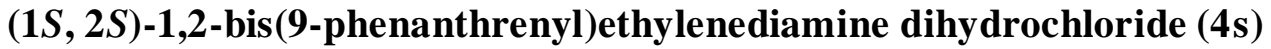

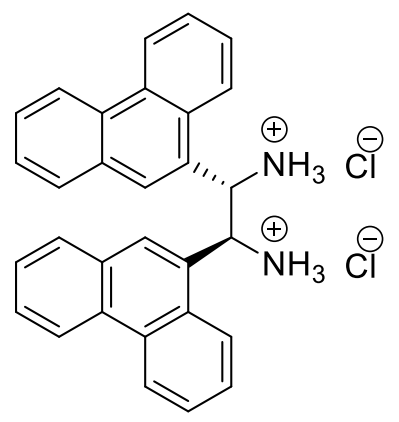

Prepared according to general procedure from $1(50 \mathrm{mg}, 0.20 \mathrm{mmol})$ and 9 -

phenanthrenecarboxaldehyde (2s) (89 $\mathrm{mg}, 0.43 \mathrm{mmol})$; washed with DCM, EA and THF to give the title compound as a white solid $(64 \mathrm{mg}, 65 \%)$.

${ }^{1} \mathrm{H}$ NMR (DMSO- $\left.d_{6}, 400 \mathrm{MHz}, 25^{\circ} \mathrm{C}\right): \delta 9.56(\mathrm{~s}, 6 \mathrm{H}), 8.70(\mathrm{~s}, 2 \mathrm{H}), 8.63-8.43(\mathrm{~m}, 6 \mathrm{H}), 7.74-$ $7.62(\mathrm{~m}, 4 \mathrm{H}), 7.50(\mathrm{~m}, 6 \mathrm{H}), 6.51(\mathrm{~s}, 2 \mathrm{H})$.

${ }^{13} \mathrm{C}\left\{{ }^{1} \mathrm{H}\right\}$ NMR (DMSO- $\left.d_{6}, 101 \mathrm{MHz}, 25{ }^{\circ} \mathrm{C}\right) \delta 129.70,129.45,129.41,129.23,128.99,128.40$, $128.00,127.62,127.05,126.81,126.69,123.98,123.26,122.58,51.70$.

HRMS (ESI) calculated for $\mathrm{C}_{30} \mathrm{H}_{25} \mathrm{~N}_{2},[\mathrm{M}+\mathrm{H}]^{+}$: 413.2012 , Found: 413.2003 .

HPLC analysis condition:

Solvent: $n$-hexane/ iso-propanol $=85: 15$

Column: CHIRALPAK IC column (Nomura Chemical Co., Ltd.; inner diameter $4.6 \mathrm{~mm} \times$ length $250 \mathrm{~mm}$; particle size $=5 \mu \mathrm{m}$ )

Column temperature: $25{ }^{\circ} \mathrm{C}$

Detector: UV at $254 \mathrm{~nm}$

Flow rate: $0.8 \mathrm{~mL} / \mathrm{min}$ Injection volume: $5 \mu \mathrm{L}$ $(R, R)-\mathbf{4} \mathbf{s} \mathrm{t}_{\mathrm{R}}=12.8 \mathrm{~min},(S, S)-\mathbf{4} \mathbf{s} \mathrm{t}_{\mathrm{R}}=14.7 \mathrm{~min}$ for racemic sample of $\mathbf{4 s}$.

ee $>99 \%$ 


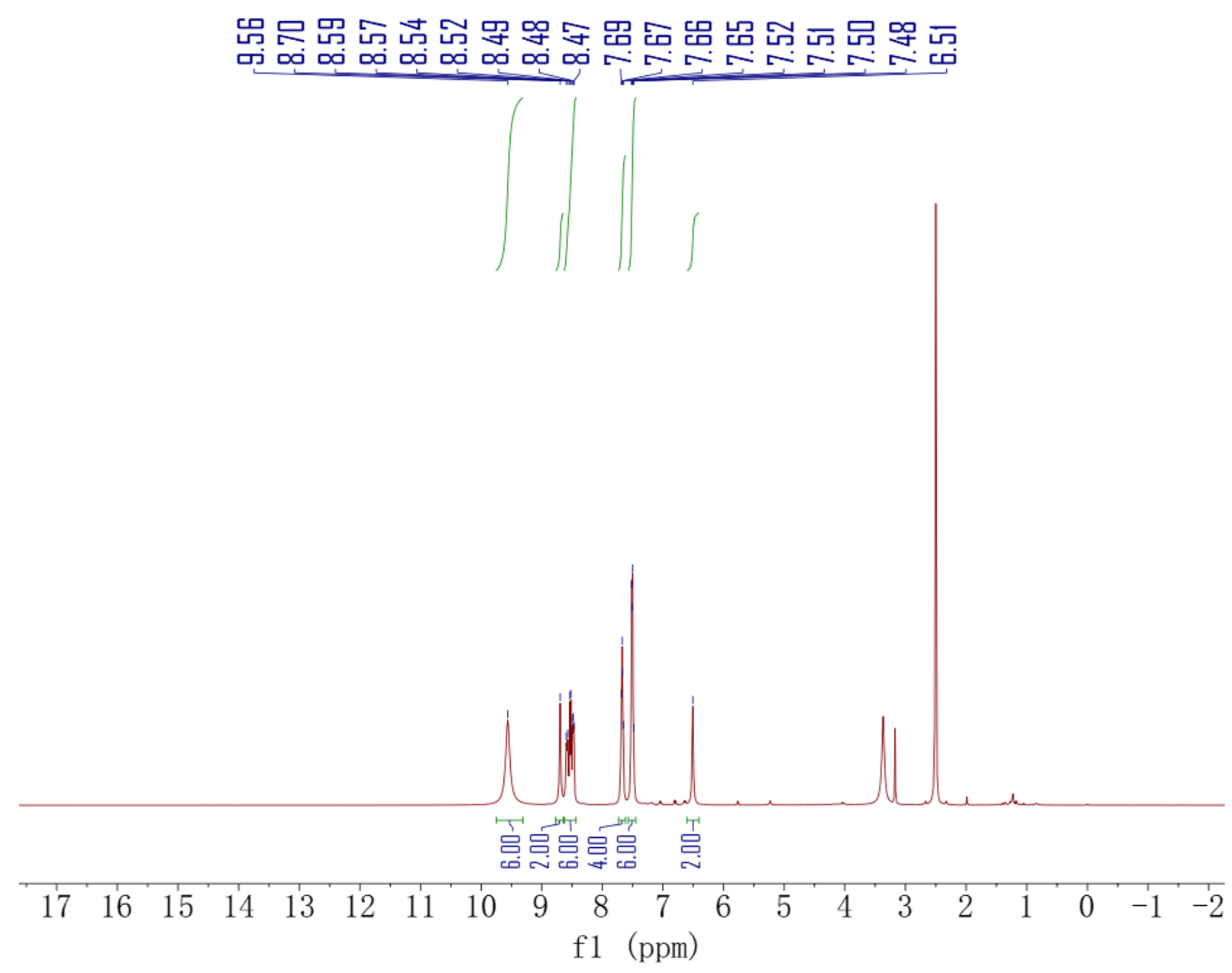

Figure S57. ${ }^{1} \mathrm{H}$ NMR of 4 s.

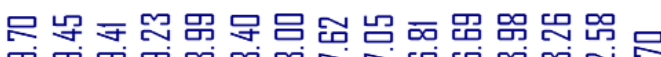

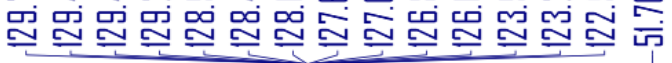

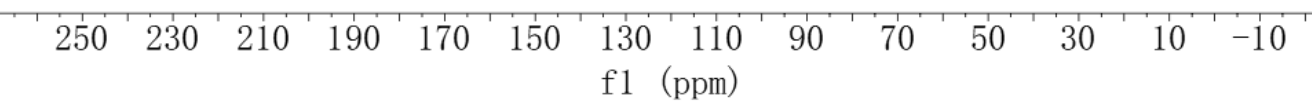

Figure S58. ${ }^{13} \mathrm{C}\left\{{ }^{1} \mathrm{H}\right\}$ NMR of 4 s. 


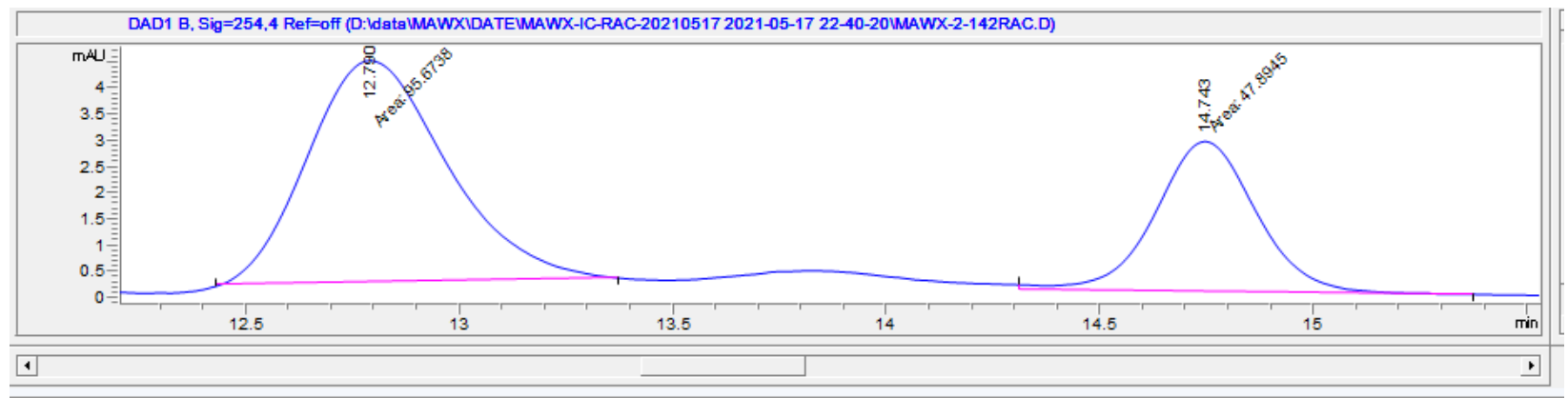

\begin{tabular}{|c|c|c|c|c|c|c|} 
\# & \multicolumn{1}{c}{ Time } & Area & Height & Width & \multicolumn{2}{c|}{ Area\% } \\
\hline 1 & 12.79 & 95.7 & 4.2 & 0.3753 & 66.640 & 0.795 \\
\hline 2 & 14.743 & 47.9 & 2.9 & 0.2769 & 33.360 & 0.937 \\
\hline
\end{tabular}

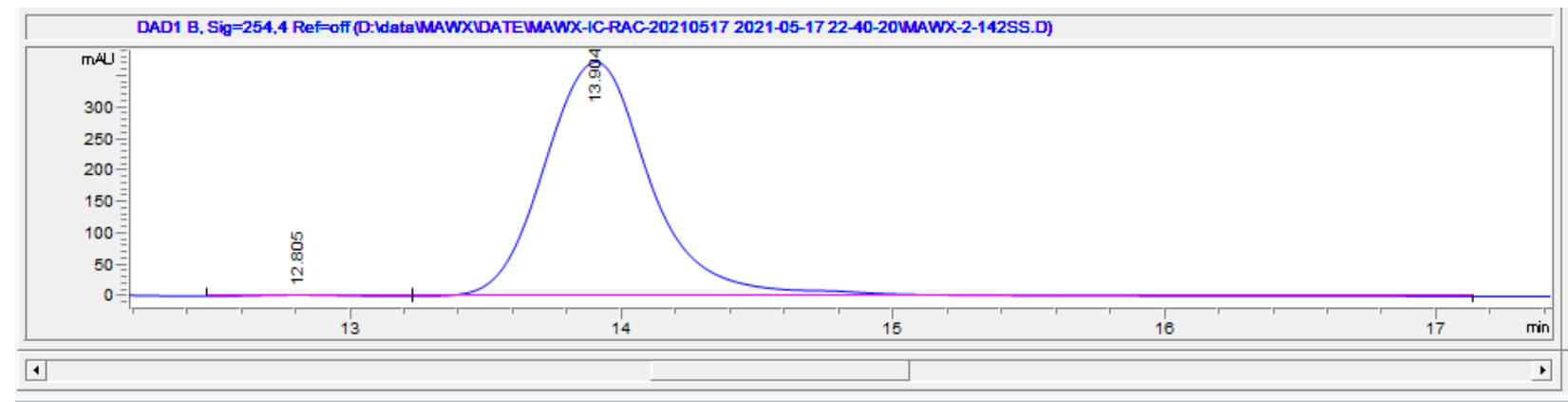

\begin{tabular}{|c|c|c|c|c|c|c|}
\multicolumn{1}{c}{ Time } & \multicolumn{1}{c}{ Area } & Height & Width & \multicolumn{2}{c|}{ Area\% } & \multicolumn{1}{c}{ Symmetry } \\
\hline 1 & 12.805 & 38.3 & 1.9 & 0.3028 & 0.374 & 0.865 \\
\hline 2 & 13.904 & 10212.4 & 371.8 & 0.4205 & 99.626 & 0.853 \\
\hline
\end{tabular}

Figure S59. HPLC analysis of (top) racemic sample, and (bottom) $(S, S)$ sample of $\mathbf{4 s .}$ 


\section{$(1 S, 2 S)$-1,2-bis(1-pyrenyl)ethylenediamine dihydrochloride (4t)}

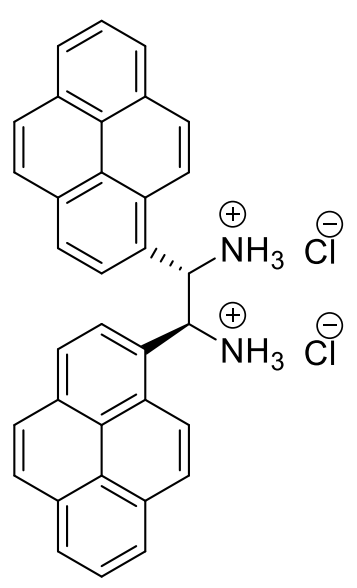

Prepared according to general procedure from 1 (50 $\mathrm{mg}, 0.20 \mathrm{mmol})$ and 1 pyrenecarboxaldehyde (2t) (100 mg, $0.43 \mathrm{mmol}$ ); washed with DCM, EA and THF to give the title compound as a white solid (104 $\mathrm{mg}, 96 \%)$.

${ }^{1} \mathrm{H}$ NMR (DMSO- $\left.d_{6}, 400 \mathrm{MHz}, 25^{\circ} \mathrm{C}\right): \delta 9.84(\mathrm{~s}, 6 \mathrm{H}), 8.83(\mathrm{~m}, 4 \mathrm{H}), 8.38(\mathrm{~d}, J=9.4 \mathrm{~Hz}, 2 \mathrm{H})$, $8.29(\mathrm{~d}, J=7.7 \mathrm{~Hz}, 2 \mathrm{H}), 8.11(\mathrm{~d}, J=7.6 \mathrm{~Hz}, 2 \mathrm{H}), 7.94(\mathrm{~m}, 6 \mathrm{H}), 7.73(\mathrm{~d}, J=8.8 \mathrm{~Hz}, 2 \mathrm{H}), 7.01$ (s, $2 \mathrm{H})$.

${ }^{13} \mathrm{C}\left\{{ }^{1} \mathrm{H}\right\}$ NMR (DMSO- $\left.d_{6}, 101 \mathrm{MHz}, 25{ }^{\circ} \mathrm{C}\right) \delta 130.63,130.42,129.78,128.55,128.33,127.96$, $127.94,126.81,126.47,125.82,125.53,125.52,124.59,123.29,123.24,122.74,52.61$.

HRMS (ESI) calculated for $\mathrm{C}_{34} \mathrm{H}_{25} \mathrm{~N}_{2},[\mathrm{M}+\mathrm{H}]^{+}:$461.2012, Found: 461.1998 .

HPLC analysis condition:

Solvent: $n$-hexane/ iso-propanol $=85: 15$

Column: CHIRALPAK IC-H column (Nomura Chemical Co., Ltd.; inner diameter $4.6 \mathrm{~mm} \times$ length $250 \mathrm{~mm}$; particle size $=5 \mu \mathrm{m}$ )

Column temperature: $25{ }^{\circ} \mathrm{C}$

Detector: UV at $254 \mathrm{~nm}$

Flow rate: $0.8 \mathrm{~mL} / \mathrm{min}$

Injection volume: $5 \mu \mathrm{L}$

$(S, S)-\mathbf{4 t} \mathrm{t}_{\mathrm{R}}=19.2 \mathrm{~min},(R, R)-\mathbf{4 t} \mathrm{t}_{\mathrm{R}}=23.9 \mathrm{~min}$ for racemic sample of $\mathbf{4 t}$.

ee $>99 \%$ 

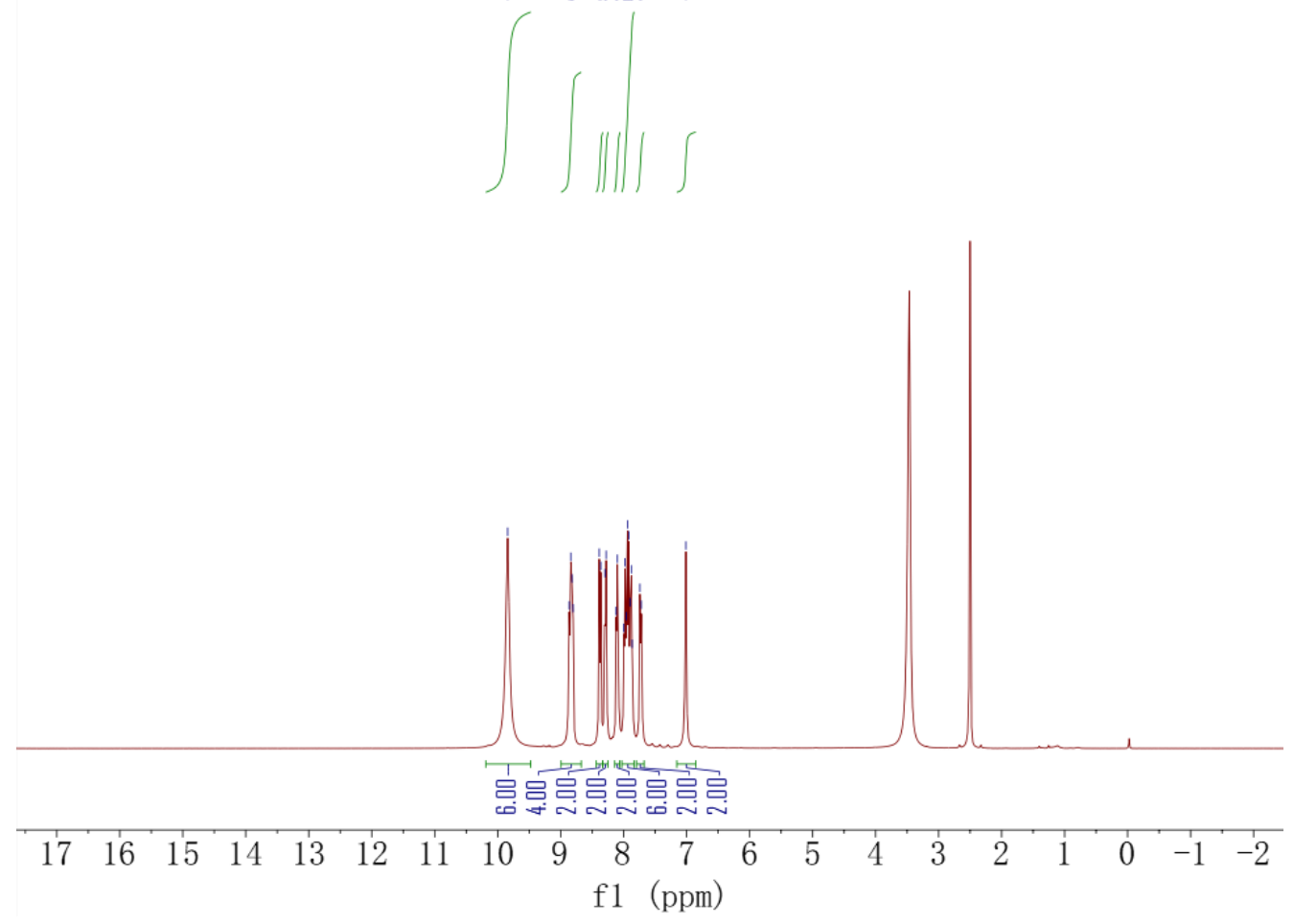

Figure S60. ${ }^{1} \mathrm{H}$ NMR of $\mathbf{4 t}$.

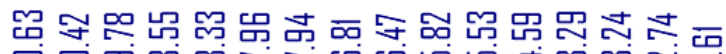

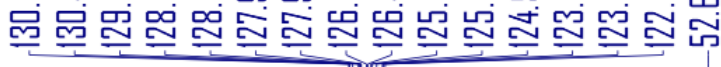

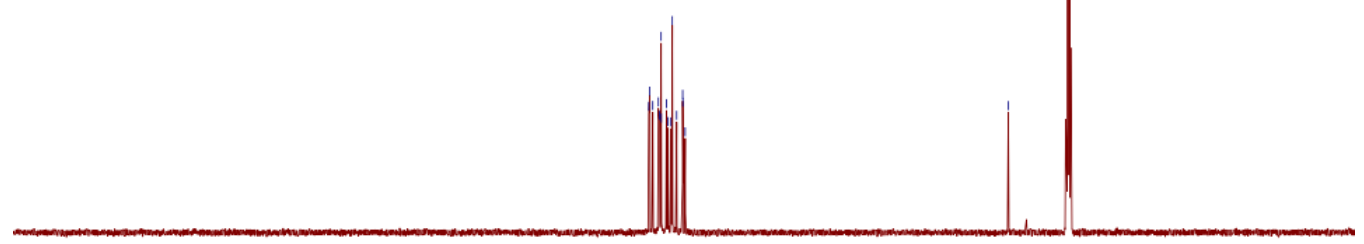

$\begin{array}{lllllllllllll}250 & 230 & 210 & 190 & 170 & 150 & \begin{array}{l}130 \\ \mathrm{f} 1\end{array}\left(\begin{array}{lllll}110 \\ (\mathrm{ppm})\end{array}\right. & 90 & 70 & 50 & 30 & 10 & -10\end{array}$

Figure S61. ${ }^{13} \mathrm{C}\left\{{ }^{1} \mathrm{H}\right\}$ NMR of $\mathbf{4 t}$. 

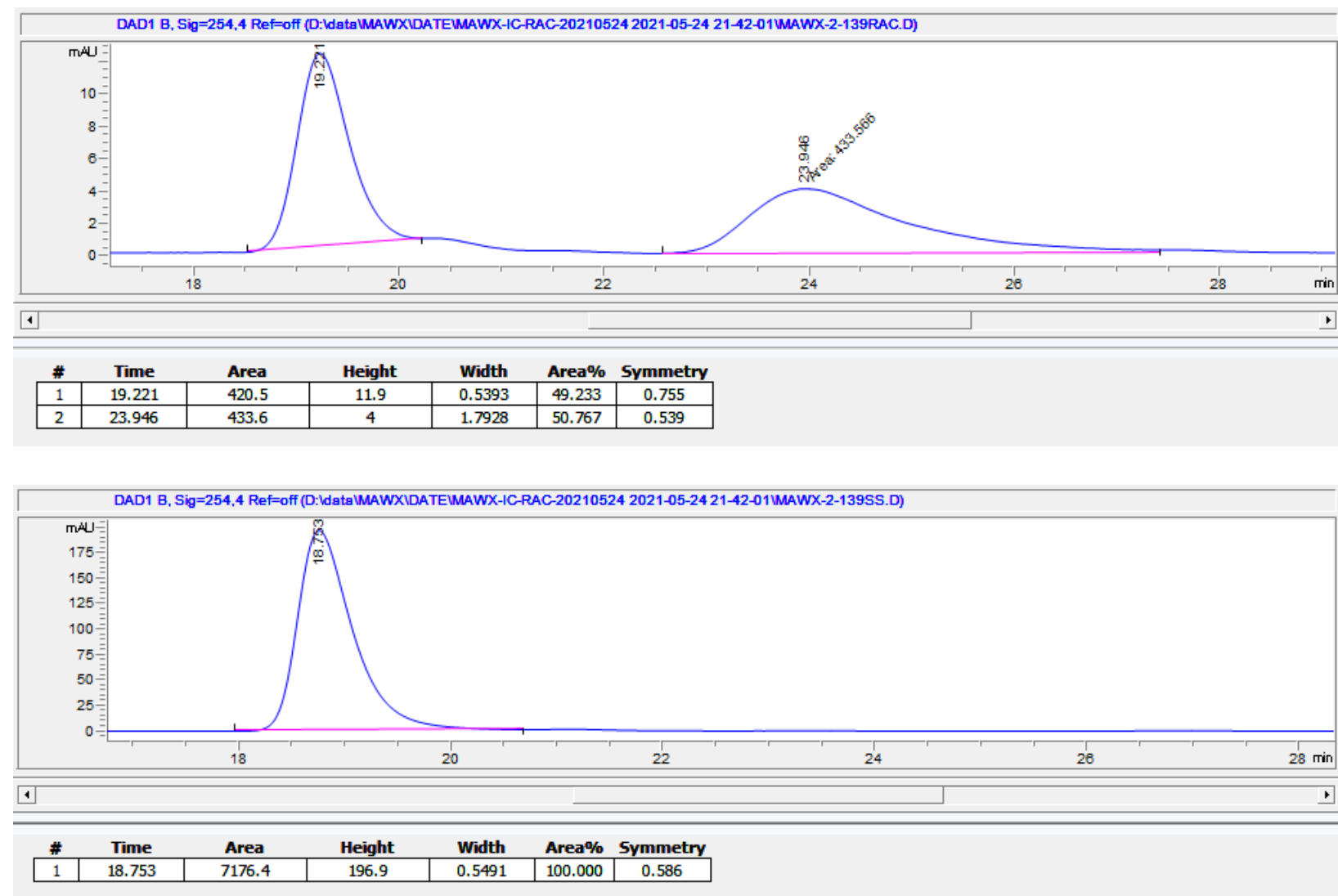

Figure S62. HPLC analysis of (top) racemic sample, and (bottom) $(S, S)$ sample of $\mathbf{4 t}$. 
4. Substrates tested that are not suitable for mechanochemical diaza-Cope rearrangement NOT WORKING<smiles>CC(C)(C)c1cc(O)c(C=O)c(C(C)(C)C)c1</smiles><smiles>O=Cc1ccc(OC2OC(CO)C(O)[C@H](O)[C@H]2O)cc1</smiles>
80154-34-3<smiles>CC(C)(C)c1cc(C=O)c(O)c(C(C)(C)Br)c1</smiles><smiles>CC(Br)(Br)c1cccc(C=O)c1O</smiles><smiles>COc1ccc(O)c(C=O)c1</smiles><smiles>O=CC1=Cc2ccccc2-c2cccc3cccc1c23</smiles>

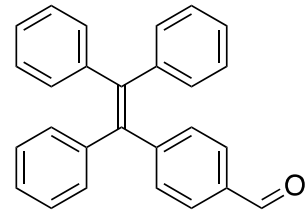<smiles>CC(C)C=O</smiles><smiles>CCCCCCCCCC=O</smiles><smiles>O=CC1CCCCC1</smiles><smiles>CCOC(=O)C(=O)CC</smiles>

Figure S63. Substrates that do not work under our solvent-free mechanochemical diaza-Cope rearrangement protocol. 
5. General procedure of solvent-free mechanochemical one-pot two-step chiral Zn salen complexes<smiles>NC(c1ccccc1O)[C@H](N)c1ccccc1O</smiles>

$(R, R) 1$<smiles>[X]c1ccc(C=O)cc1</smiles>

2.1 equiv.

2

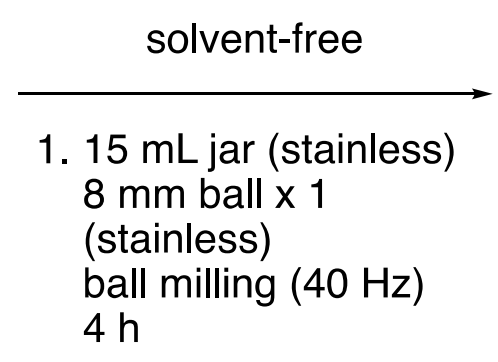

2. $\mathrm{Zn}(\mathrm{OAc})_{2}$ (1.5 equiv) ball milling $(40 \mathrm{~Hz})$ $2 \mathrm{~h}$

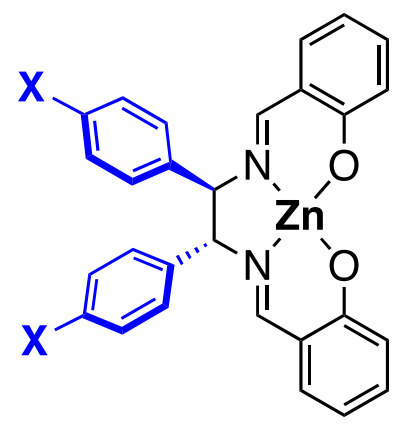

$1(0.20 \mathrm{mmol})$, and $2(0.42 \mathrm{mmol}, 2.1$ equiv.) were placed in a $15 \mathrm{ml}$ stainless steel ball milling vessel (JX-KG0180) loaded with one grinding ball [stainless steel (JX-GZ0142), diameter: $12 \mathrm{~mm}$ ]. After the vessel was closed in air, the vessel was placed in the ball mill machine. After $4 \mathrm{~h}$ of ballmilling at $40 \mathrm{~Hz}$, with a periodic $5 \mathrm{~min}$ pause for every $1 \mathrm{~h}$ of milling, then open the vessel, add $\mathrm{Zn}(\mathrm{OAc}) \cdot 2\left(\mathrm{H}_{2} \mathrm{O}\right)(0.30 \mathrm{mmol}, 1.5$ equiv. $)$, continue to grind the mixture at $40 \mathrm{~Hz}$ for 2 hours, the solid residue is dissolved in $10 \mathrm{~mL}$ methanol, then $20 \mathrm{~mL}$ water is added to it, and the product will precipitate out as a pale-yellow powder, the solid is obtained by filtration, and the filter cake is washed several times with a mixed solvent of 1:2 ratio of $\mathrm{MeOH}$ :water. The volatile was evaporated under reduced pressure to obtain analytically pure 9 . 


\section{$(1 S, 2 S)-Z n(9 b)$}

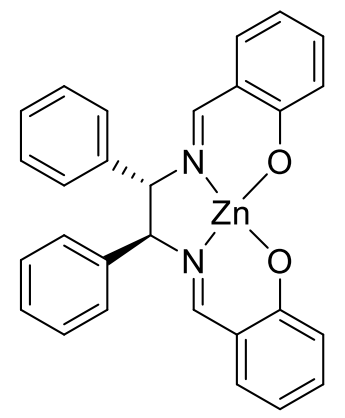

Prepared according to general procedure above from 1 (50 $\mathrm{mg}, 0.20 \mathrm{mmol}$ ) and benzaldehyde (2b) (45 mg, $0.43 \mathrm{mmol})$, and zinc acetate dihydrate $(67 \mathrm{mg}, 0.30 \mathrm{mmol})$ to give the title compound as a light-yellow solid (77 $\mathrm{mg}, 78 \%)$.

${ }^{1} \mathrm{H}$ NMR (DMSO- $\left.d_{6}, 400 \mathrm{MHz}, 25{ }^{\circ} \mathrm{C}\right): \delta 8.22(\mathrm{~s}, 2 \mathrm{H}), 7.39(\mathrm{~d}, J=7.7 \mathrm{~Hz}, 4 \mathrm{H}), 7.34(\mathrm{t}, J=7.5$ $\mathrm{Hz}, 4 \mathrm{H}), 7.26(\mathrm{t}, J=7.2 \mathrm{~Hz}, 2 \mathrm{H}), 7.15(\mathrm{~m}, 2 \mathrm{H}), 7.00(\mathrm{dd}, J=7.8,1.9 \mathrm{~Hz}, 2 \mathrm{H}), 6.65(\mathrm{~d}, J=8.5$ $\mathrm{Hz}, 2 \mathrm{H}), 6.37$ (t, $J=7.3 \mathrm{~Hz}, 2 \mathrm{H}), 5.09$ (s, $2 \mathrm{H})$.

${ }^{13} \mathrm{C}\left\{{ }^{1} \mathrm{H}\right\}$ NMR (DMSO- $\left.d_{6}, 101 \mathrm{MHz}, 25{ }^{\circ} \mathrm{C}\right) \delta 171.33,169.73,141.08,135.12,133.39,128.50$, $127.75,127.51,122.77,119.23,112.36,72.34$.

HRMS (ESI) calculated for $\mathrm{C}_{28} \mathrm{H}_{23} \mathrm{~N}_{2} \mathrm{O}_{2} \mathrm{Zn}^{+},[\mathrm{M}+\mathrm{H}]^{+}: 483.1069$, Found: 483.1027. 


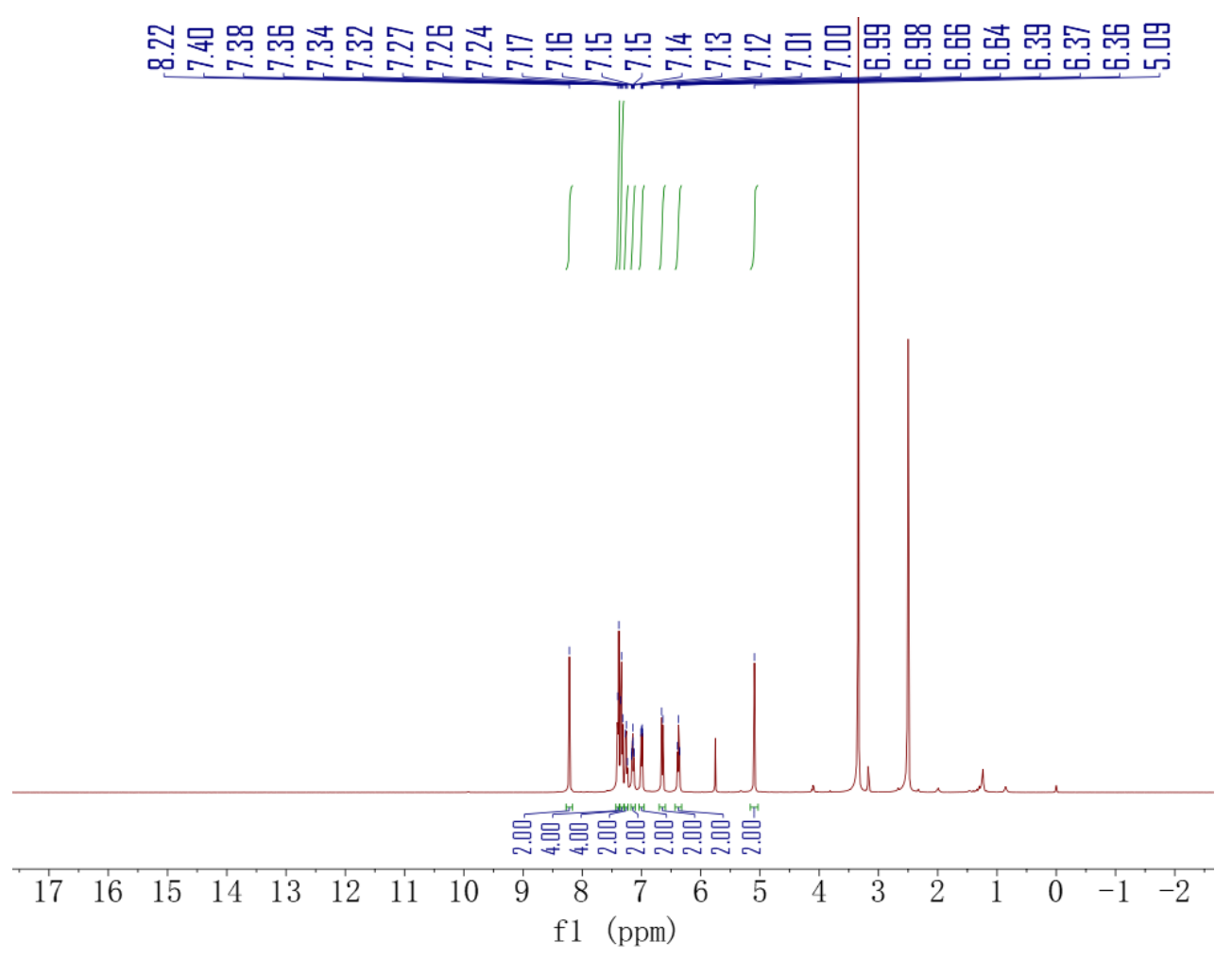

Figure S64. ${ }^{1} \mathrm{H}$ NMR of $9 b$.

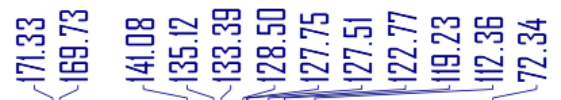

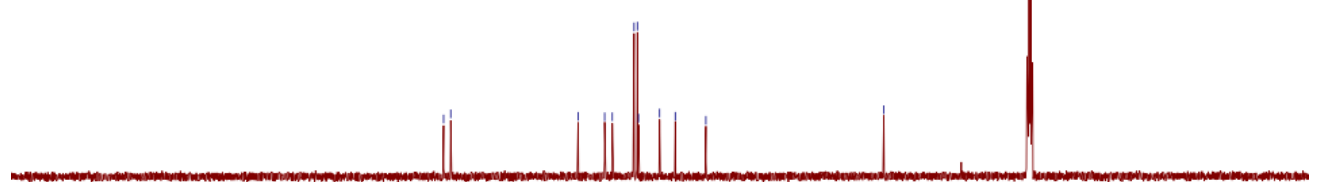

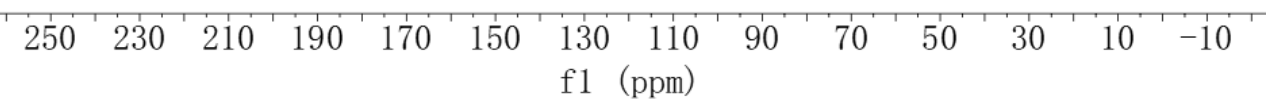

Figure S65. ${ }^{13} \mathrm{C}\left\{{ }^{1} \mathrm{H}\right\} \mathrm{NMR}$ of $\mathbf{9 b}$. 


\section{$(1 S, 2 S)-Z n(9 \mathrm{e})$}

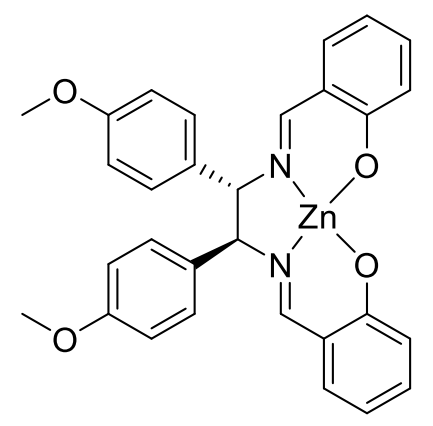

Prepared according to general procedure above from 1 (50 mg, $0.20 \mathrm{mmol})$ and 4 methoxybenzaldehyde (2b) (58 $\mathrm{mg}, 0.43 \mathrm{mmol})$, and zinc acetate dihydrate $(67 \mathrm{mg}, 0.30 \mathrm{mmol})$ to give the title compound as a light-yellow solid (78 $\mathrm{mg}, 70 \%)$.

${ }^{1} \mathrm{H}$ NMR (DMSO- $\left.d_{6}, 400 \mathrm{MHz}, 25{ }^{\circ} \mathrm{C}\right): \delta 8.22(\mathrm{~s}, 2 \mathrm{H}), 7.91(\mathrm{~d}, J=8.0 \mathrm{~Hz}, 4 \mathrm{H}), 7.57(\mathrm{~d}, J=8.1$ $\mathrm{Hz}, 4 \mathrm{H}), 7.15(\mathrm{t}, J=6.7 \mathrm{~Hz}, 2 \mathrm{H}), 7.00(\mathrm{~d}, J=7.2 \mathrm{~Hz}, 2 \mathrm{H}), 6.66(\mathrm{~d}, J=8.5 \mathrm{~Hz}, 2 \mathrm{H}), 6.37(\mathrm{t}, J$ $=7.3 \mathrm{~Hz}, 2 \mathrm{H}), 5.23(\mathrm{~s}, 2 \mathrm{H}), 3.81(\mathrm{~s}, 6 \mathrm{H})$.

${ }^{13} \mathrm{C}\left\{{ }^{1} \mathrm{H}\right\}$ NMR (DMSO- $\left.d_{6}, 101 \mathrm{MHz}, 25{ }^{\circ} \mathrm{C}\right) \delta 171.44,165.94,146.05,135.30,133.66,129.31$, $128.88,128.17,122.86,119.16,112.52,72.05,52.15$.

HRMS (ESI) calculated for $\mathrm{C}_{30} \mathrm{H}_{27} \mathrm{~N}_{2} \mathrm{O}_{4} \mathrm{Zn}^{+}$, [M+H] $]^{+}:$543.1257, Found: 543.1266. 


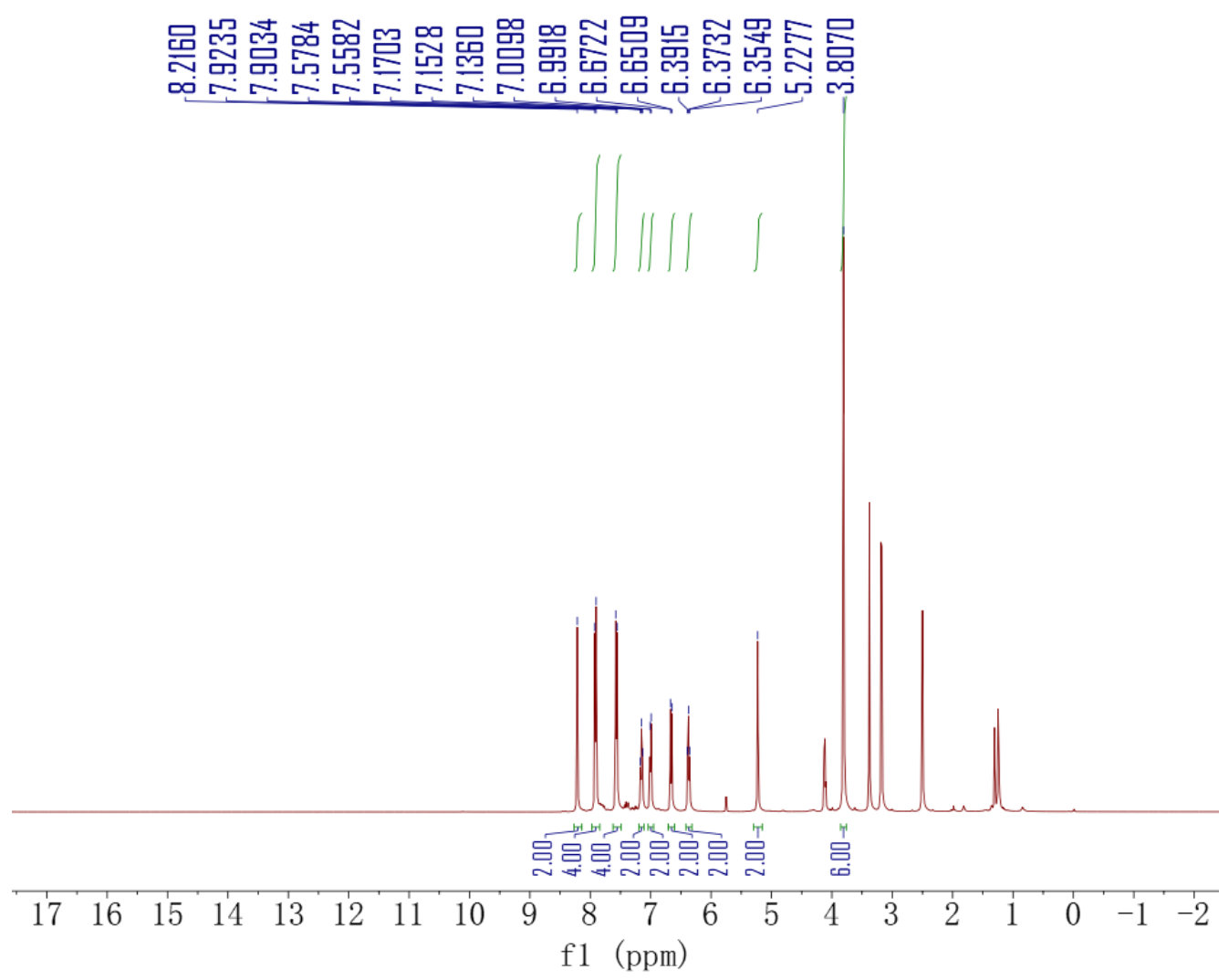

Figure S66. ${ }^{1} \mathrm{H}$ NMR of $9 e$.

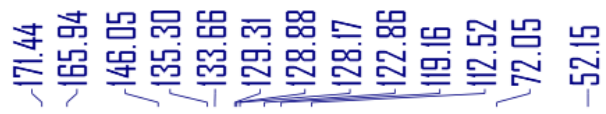
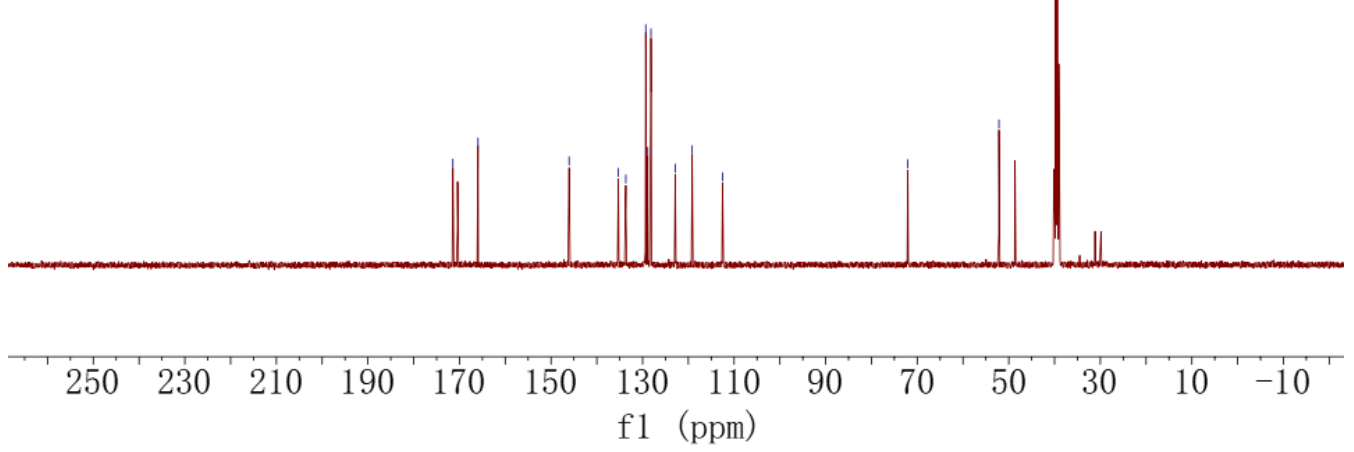

Figure S67. ${ }^{13} \mathrm{C}\left\{{ }^{1} \mathrm{H}\right\}$ NMR of $9 e$.

S76 
6. Monitoring reaction profiles of different reaction techniques with $\mathbf{1}$ and $\mathbf{2 a}$ :

a) Neat stirring (1000 rpm with one $18 \mathrm{~mm}$ stir bar)

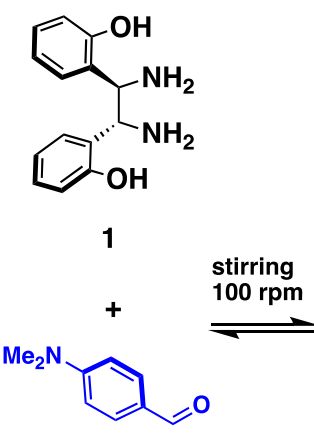

$2 a$
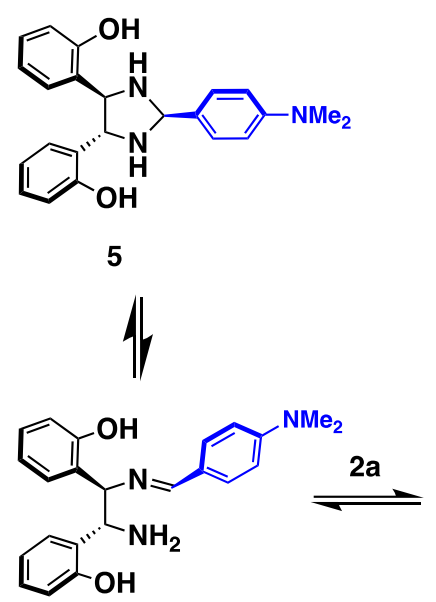

7

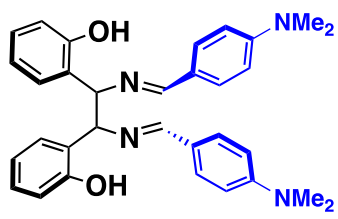

6

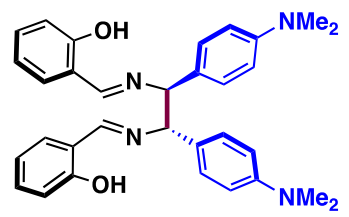

3a

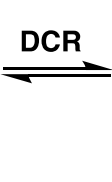

3 a

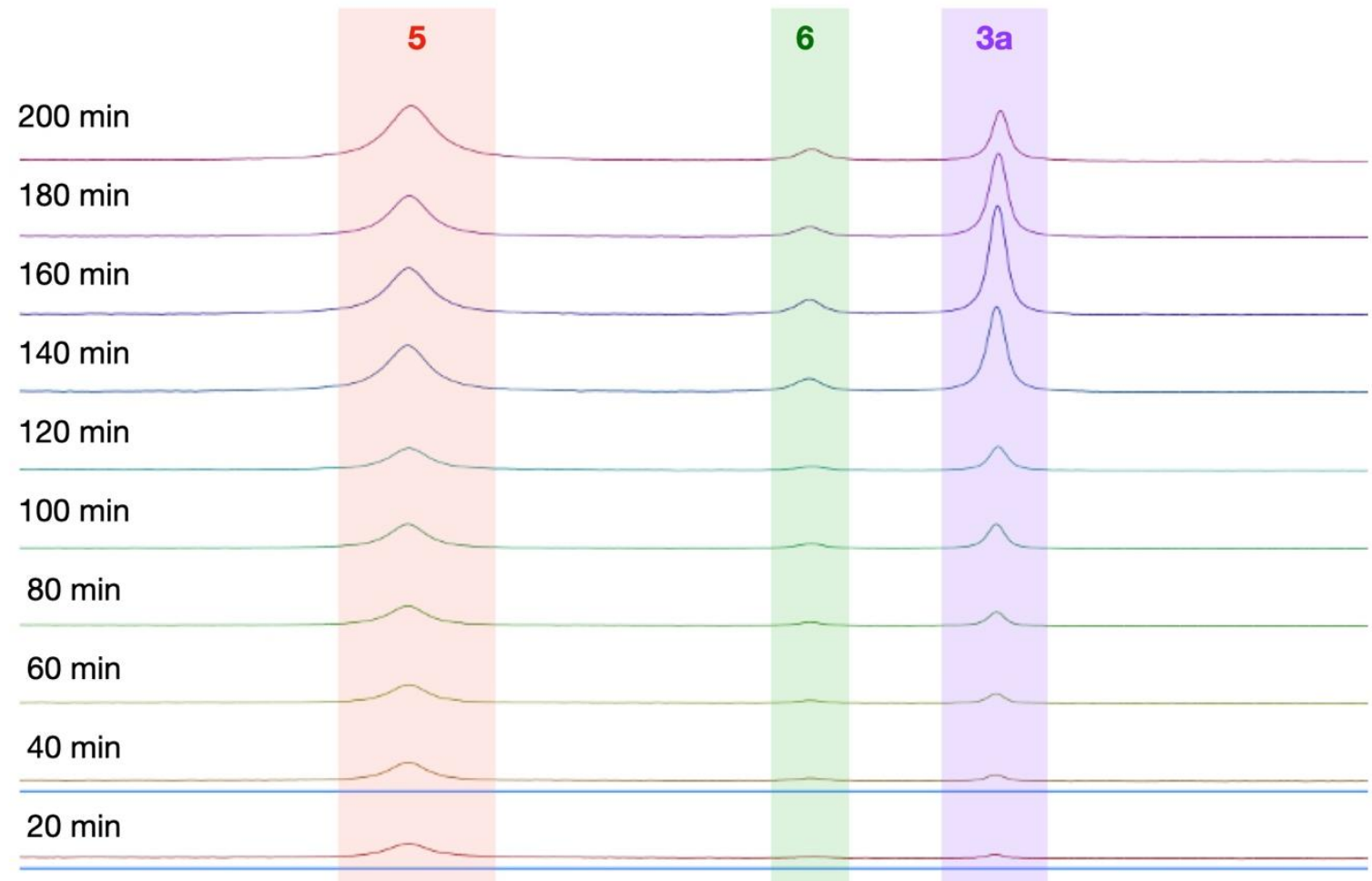
5. 16
5. 13
5. $10 \quad 5.07$
5. 04
5. 01
4. 98
4.95
4. 92
4. 89
4. 864.

Figure S68. ${ }^{1} \mathrm{H}$ NMR spectra of reaction time course monitoring on reaction of $\mathbf{1}$ and $\mathbf{2 a}$ under neat stirring condition. The stirred dry sample was dissolved in DMSO- $d_{6}$ and the NMR sample was injected into an NMR spectrometer as quickly as possible (within $2 \mathrm{~min}$ ) to minimize further solution reaction of the solid sample. After that, data acquisition began immediately to acquire the ${ }^{1} \mathrm{H}$ NMR spectrum. Ratios of different species are determined by NMR integrations against an internal standard with known concentration. 


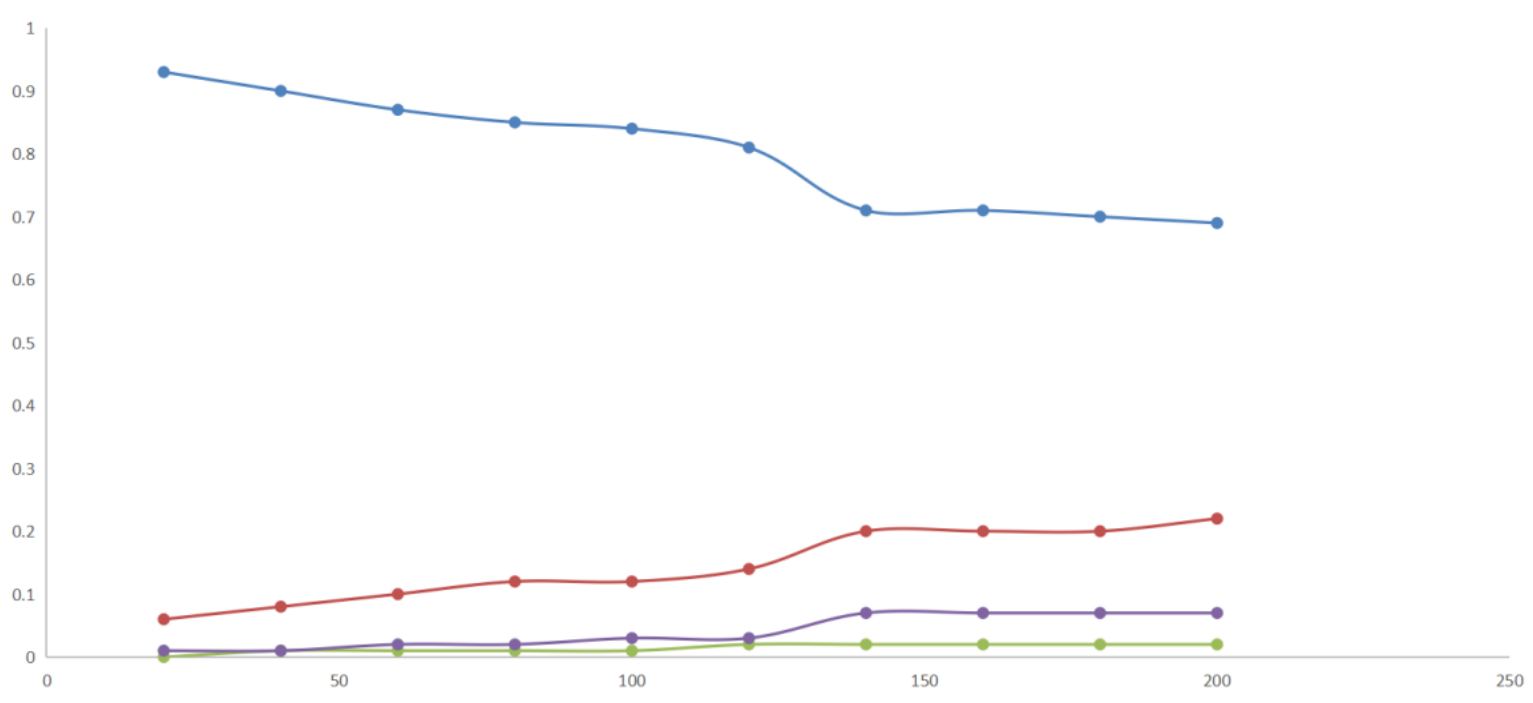

$\mathrm{X}$-axis: time in min; $\mathrm{y}$-axis: \% composition.

Blue: Mother diamine 1

Red: Five-membered ring intermediate $\mathbf{5}$

Purple: Schiff base after rearrangement 3a

Green: Schiff base before rearrangement 6

Figure S69. Plot of reaction time trace of different species: 1 (mother diamine), 5 (intermediate), and 3a (DCR product) under neat stirring condition.

Comment: Solvent-free, using magnetic stirring, under the condition of $1000 \mathrm{rpm}$, the reaction can proceed, but the reaction rate is slow. 
b) Neat sonication $\left(35 \mathrm{kHz}, 4^{\circ} \mathrm{C}\right)$

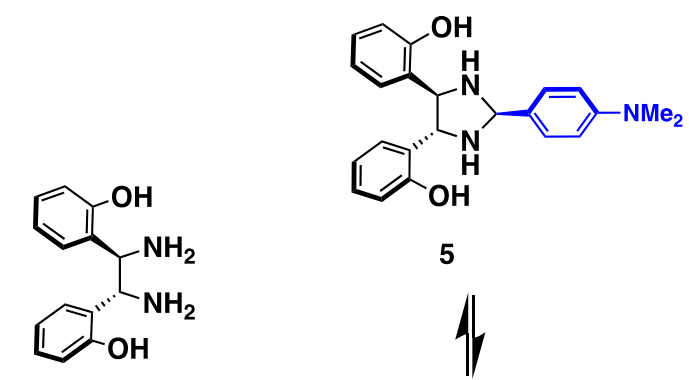

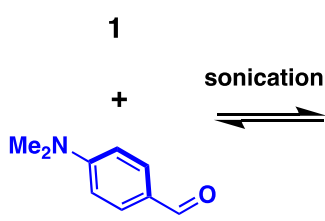

$2 a$

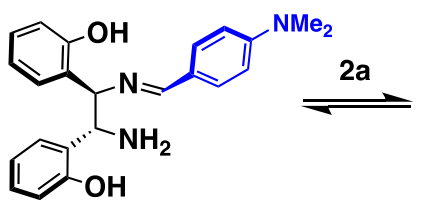

7

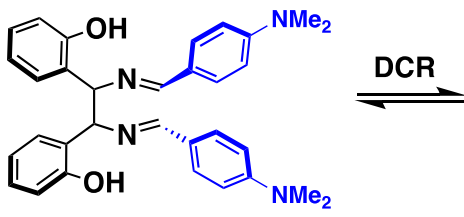

6

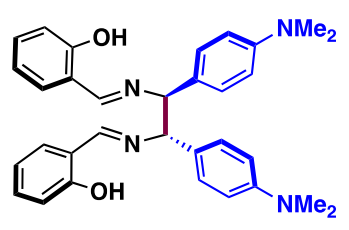

3a

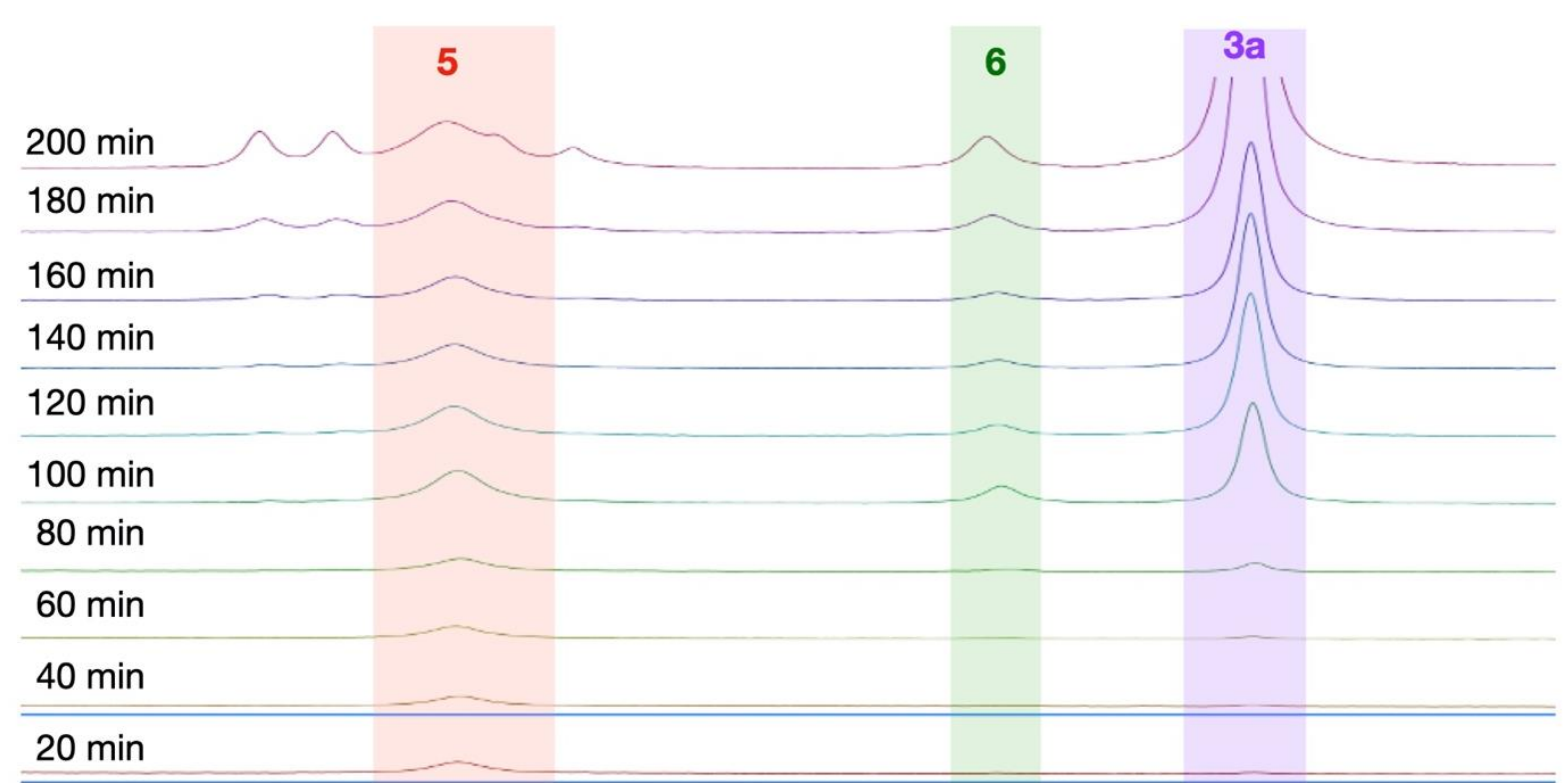

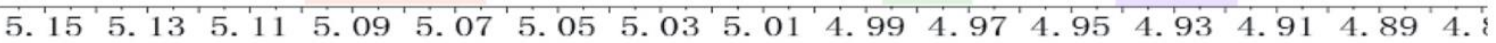

Figure S70. ${ }^{1} \mathrm{H}$ NMR spectra of reaction time course monitoring under neat sonication condition. The sonication sample was dissolved in DMSO- $d_{6}$ and the NMR sample was injected into an NMR spectrometer as quickly as possible (within $2 \mathrm{~min}$ ) to minimize further solution reaction of the sonicated sample. After that, data acquisition began immediately to acquire the ${ }^{1} \mathrm{H}$ NMR spectrum. Ratios of different species are determined by NMR integrations against an internal standard with known concentration. 


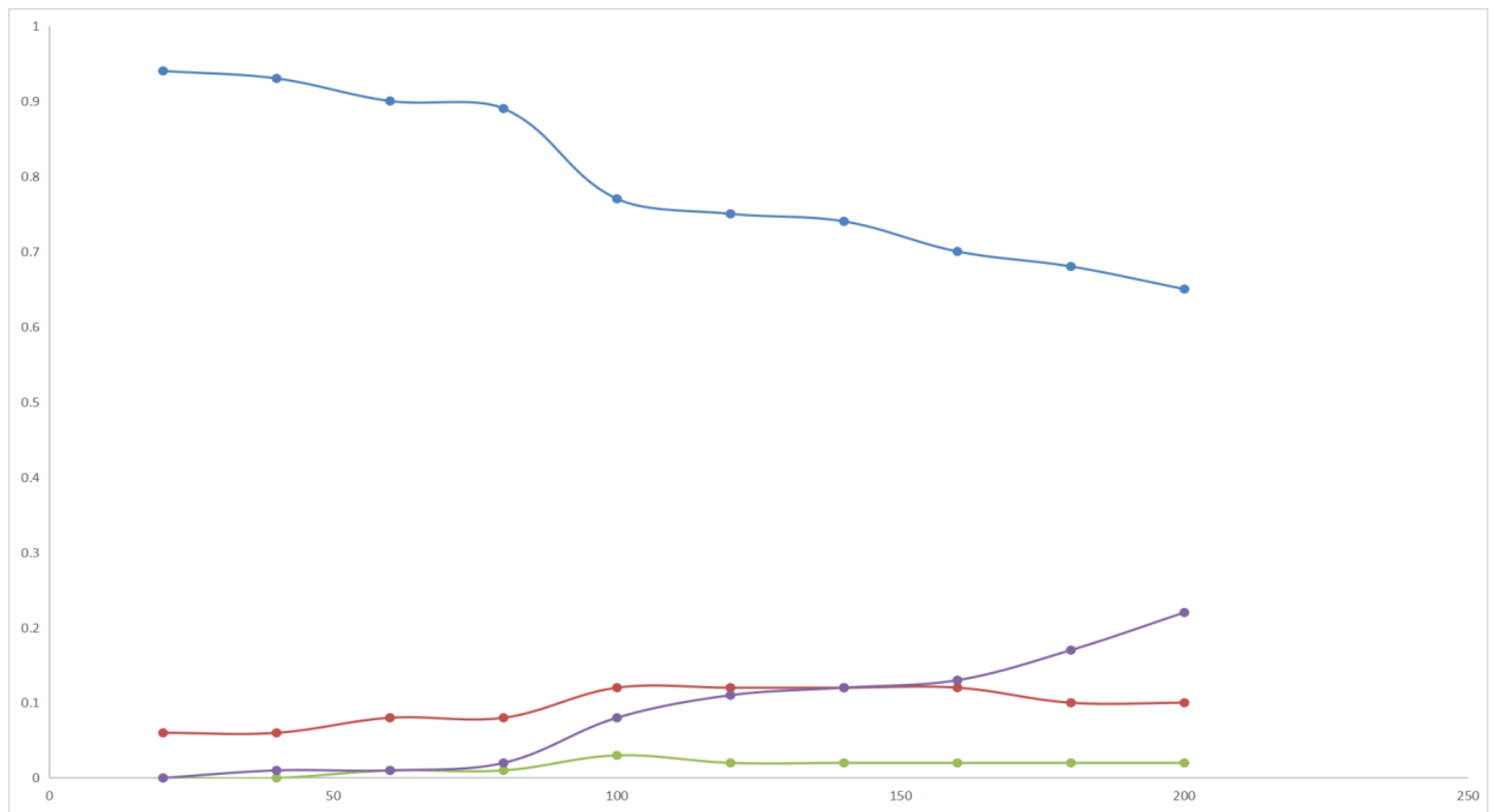

$\mathrm{X}$-axis: time in min; $\mathrm{y}$-axis: \% composition.

Blue: Mother diamine 1

Red: Five-membered ring intermediate $\mathbf{5}$

Purple: Schiff base after rearrangement 3a

Green: Schiff base before rearrangement 6

Figure S71. Plot of reaction time trace of different species: 1 (mother diamine), 5 (intermediate), and 3a (DCR product) under neat sonication condition.

Comment: Under solvent-free, sonication condition, the reaction can proceed, the speed is still $\underline{\text { slow, but faster than the neat stirring reaction condition. }}$ 
c) Solution-based condition (0.4 M in DMSO- $\left.d_{6}, 25^{\circ} \mathrm{C}\right)$

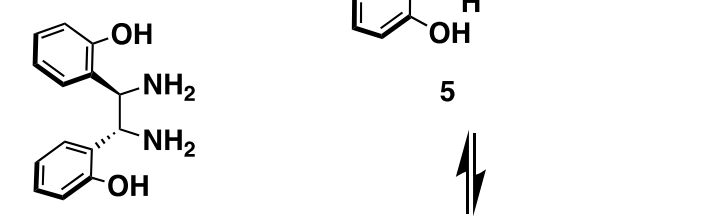

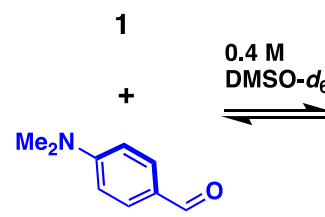

2a

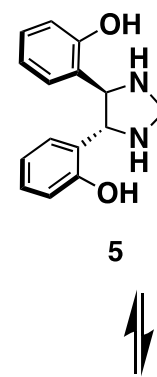

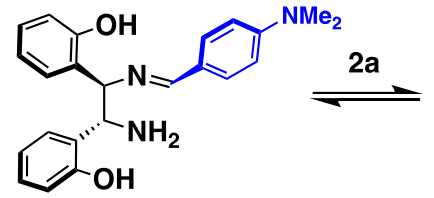

7

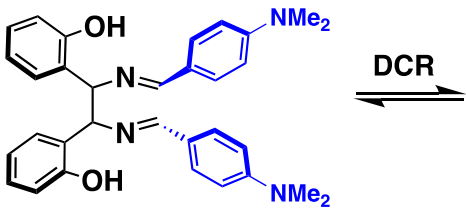

6

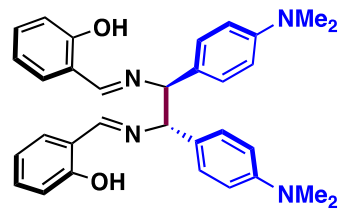

3a

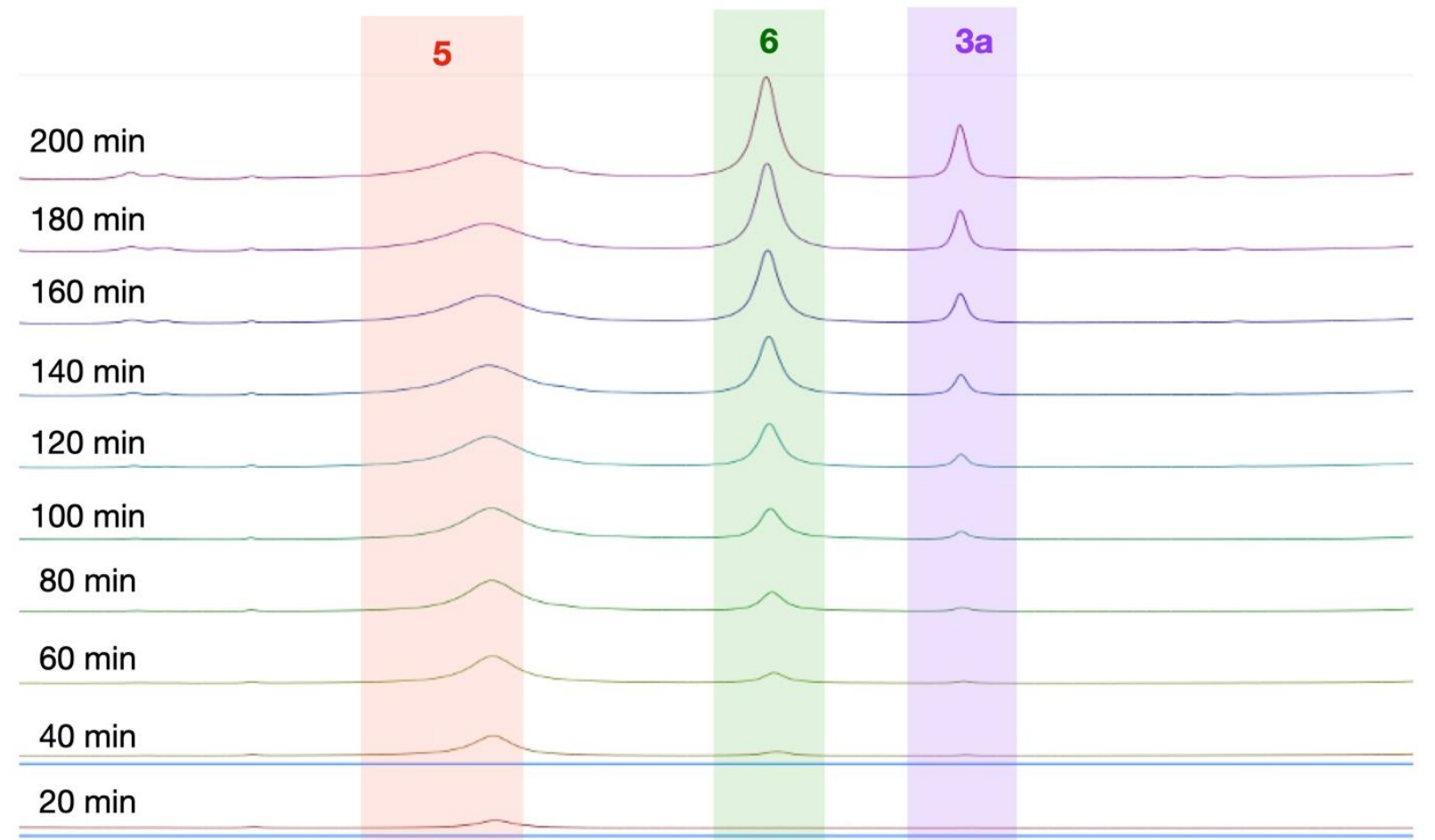

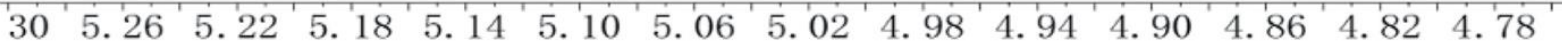

Figure S72. ${ }^{1} \mathrm{H}$ NMR spectra of reaction time course monitoring under solution condition. The NMR sample was periodically checked. Ratios of different species are determined by NMR integrations against an internal standard with known concentration. 


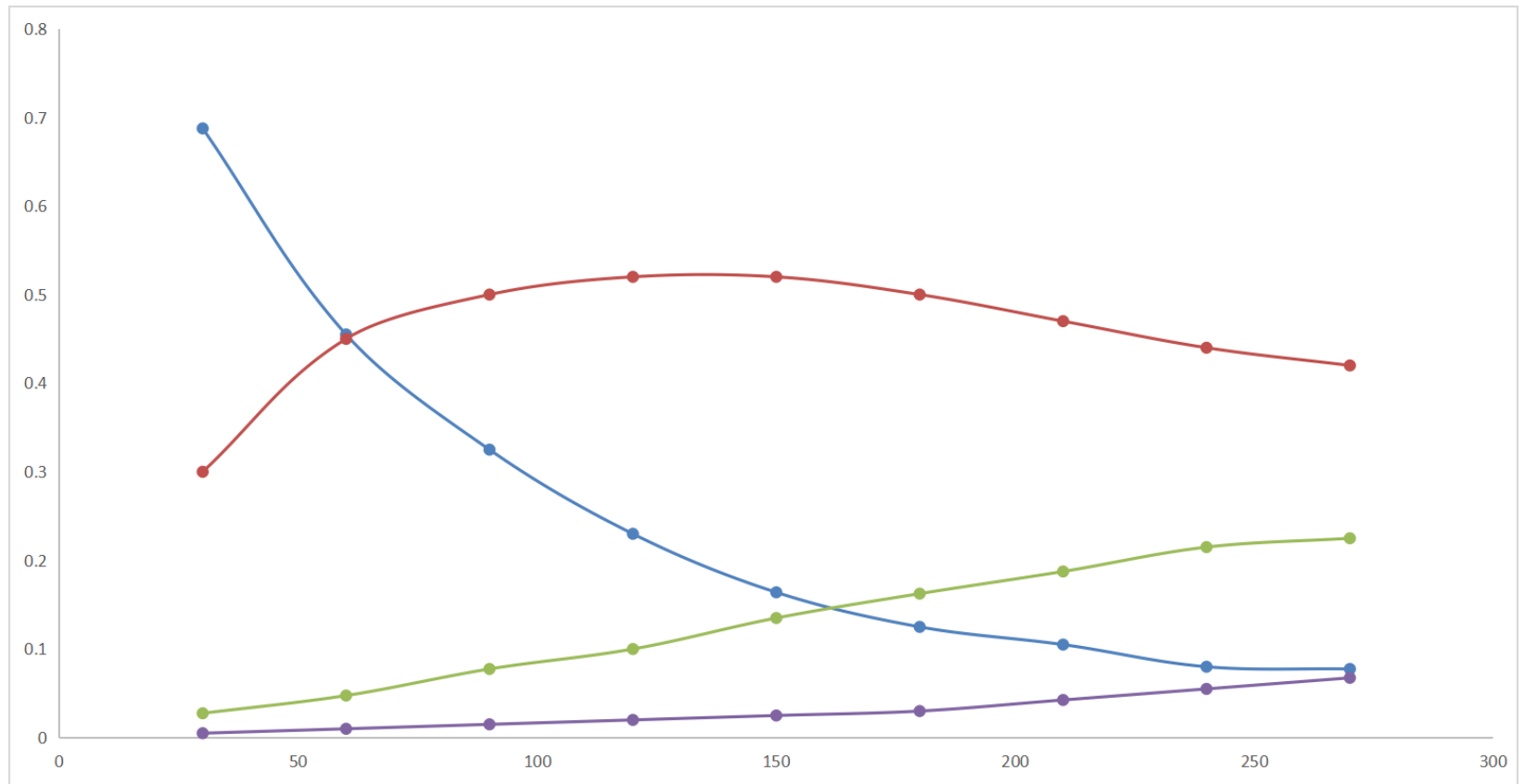

$\mathrm{X}$-axis: time in min; $\mathrm{y}$-axis: \% composition.

Blue: Mother diamine 1

Red: Five-membered ring intermediate $\mathbf{5}$

Purple: Schiff base after rearrangement 3a

Green: Schiff base before rearrangement 6

Figure S73. Plot of reaction time trace of different species: 1 (mother diamine), 5 (intermediate), and 3a (DCR product) under solution-based condition.

Comment: Under solution condition, with DMSO- $d_{6}$ as the solvent, the starting materials' concentrations are twice the concentration used by Chin. ${ }^{1}$ The mother diamine 1 conversion reaches $92 \%$ in 3 hours, but the product yield of $\mathbf{3 a}$ after rearrangement is only $6.75 \%$. 
d) Ball-milling condition $(40 \mathrm{~Hz})$

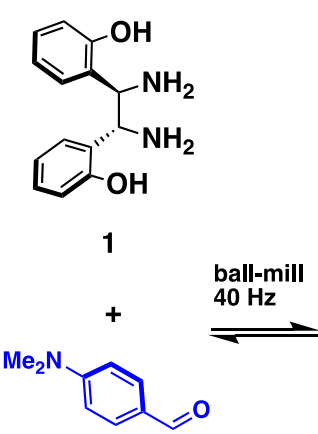

2a<smiles>CN(C)c1ccc(C2NC(c3ccccc3O)[C@H](c3ccccc3O)N2)cc1</smiles>

5

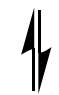

7

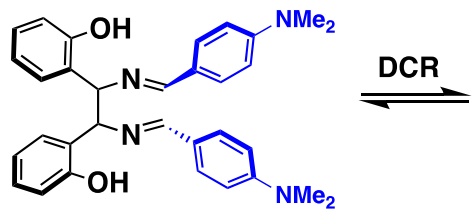

6

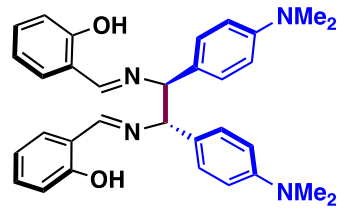

$3 a$

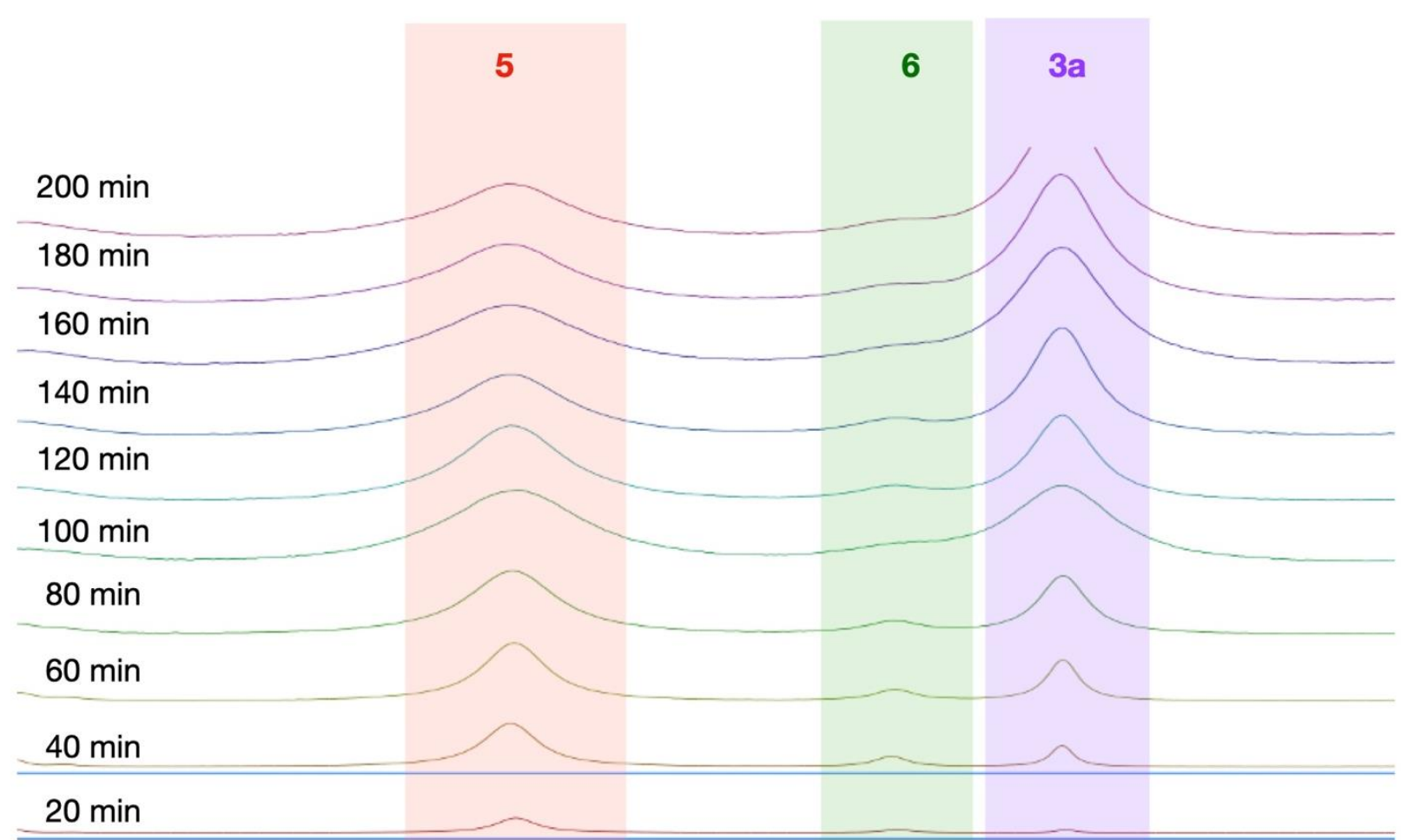
5. 17 5. 14
5. 115.08
5. 055.02
4. $99 \quad 4.96$
4. 93
4. $90 \quad 4.87 \quad 4.8$

Figure S74. ${ }^{1} \mathrm{H}$ NMR spectra of reaction time course monitoring under neat stirring condition. The milled sample was dissolved in DMSO- $d_{6}$ and the NMR sample was injected into an NMR spectrometer as quickly as possible (within $2 \mathrm{~min}$ ) to minimize further solution reaction of the milled solid sample. After that, data acquisition began immediately to acquire the ${ }^{1} \mathrm{H}$ NMR spectrum. Ratios of different species are determined by NMR integrations against an internal standard with known concentration. 


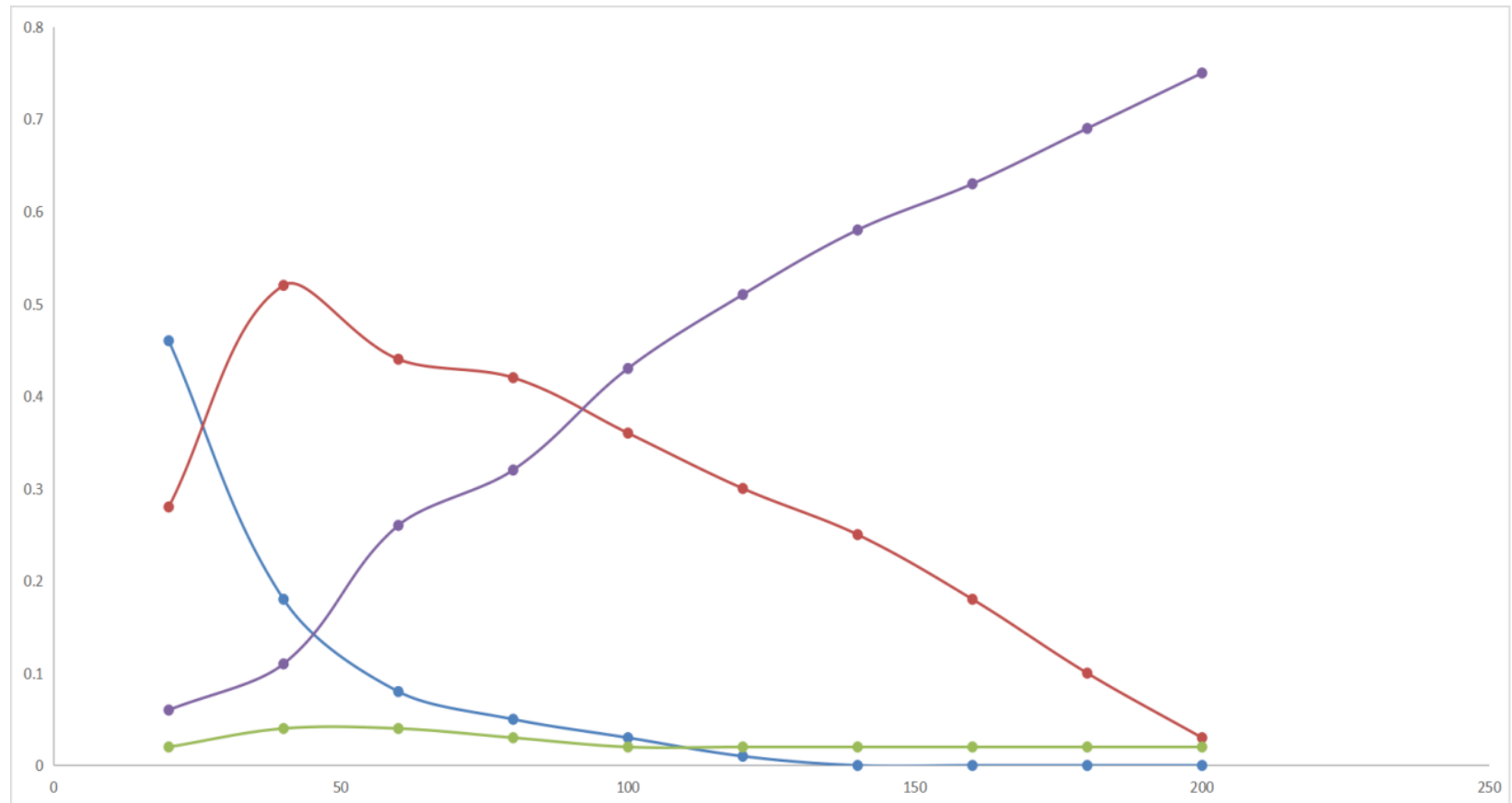

$\mathrm{X}$-axis: time in min; $\mathrm{y}$-axis: \% composition.

Blue: Mother diamine 1

Red: Five-membered ring intermediate $\mathbf{5}$

Purple: Schiff base after rearrangement 3a

Green: Schiff base before rearrangement 6

Figure S75. Plot of reaction time trace of different species: 1 (mother diamine), 5 (intermediate), and 3a (DCR product) under ball-milling condition.

Comment: Under grinding conditions, the reaction is greatly accelerated, the conversion rate of mother diamine 1 reaches $\sim 100 \%$ within $2 \mathrm{hr}$, and the yield after rearrangement 3a reaches $75 \%$ in 200 minutes. 
7. Comparison of benzocrown substrate $\mathbf{2} \mathbf{u}$ in diaza-Cope rearrangement: ball-milling vs. solution
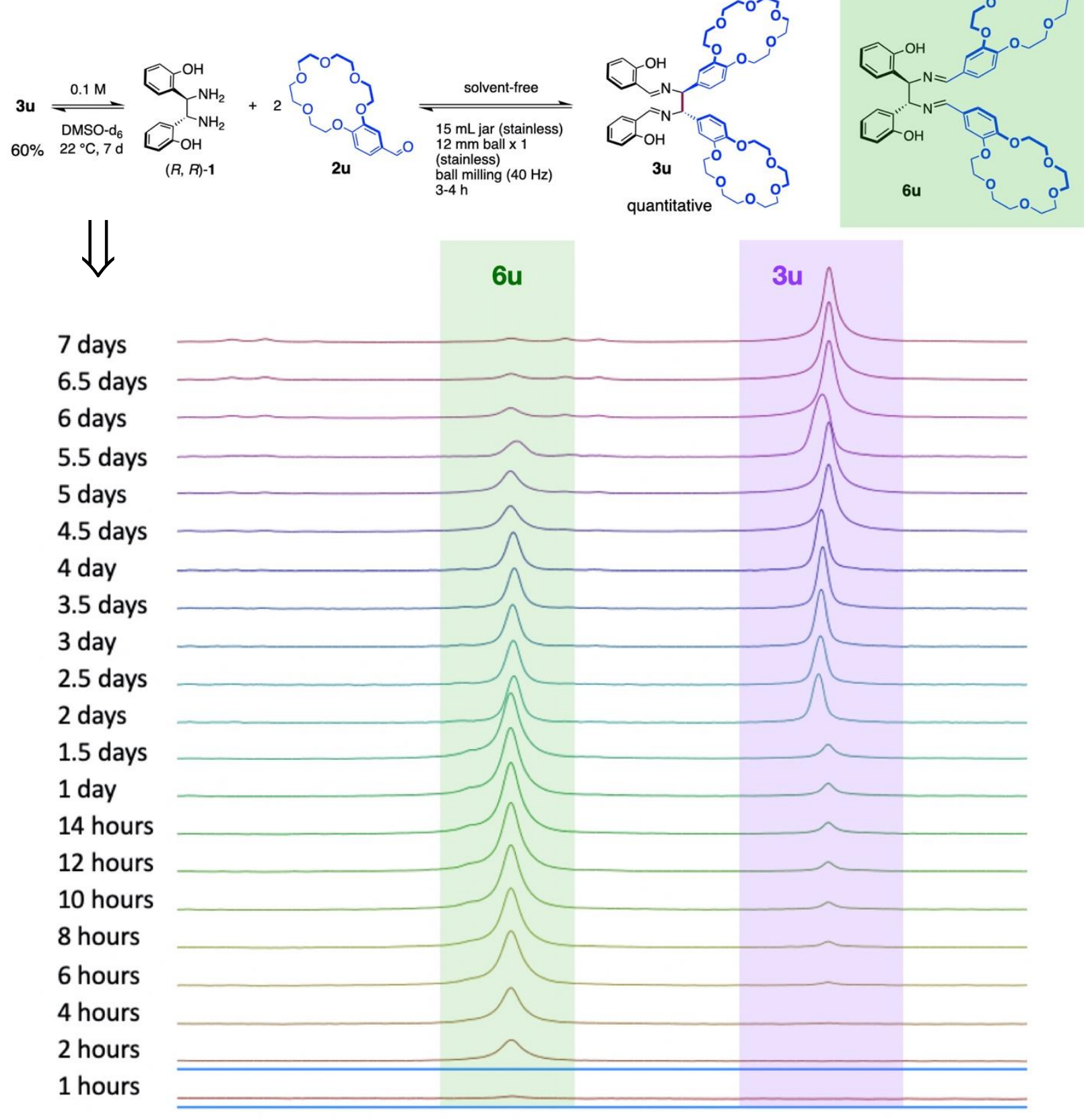

\section{$\begin{array}{lllllllllll}5.30 & 5.25 & 5.20 & 5.15 & 5.10 & 5.05 & 5.00 & 4.95 & 4.90\end{array}$}

Figure S76. ${ }^{1} \mathrm{H}$ NMR time course study of diaza-Cope rearrangement between $\mathbf{1}$ and benzocrown aldehyde $2 \mathbf{u}(0.1 \mathrm{M})$ in DMSO- $d_{6} . \%$ Yield is determined by NMR integrations against an internal standard with known concentration. 
8. PXRD studies
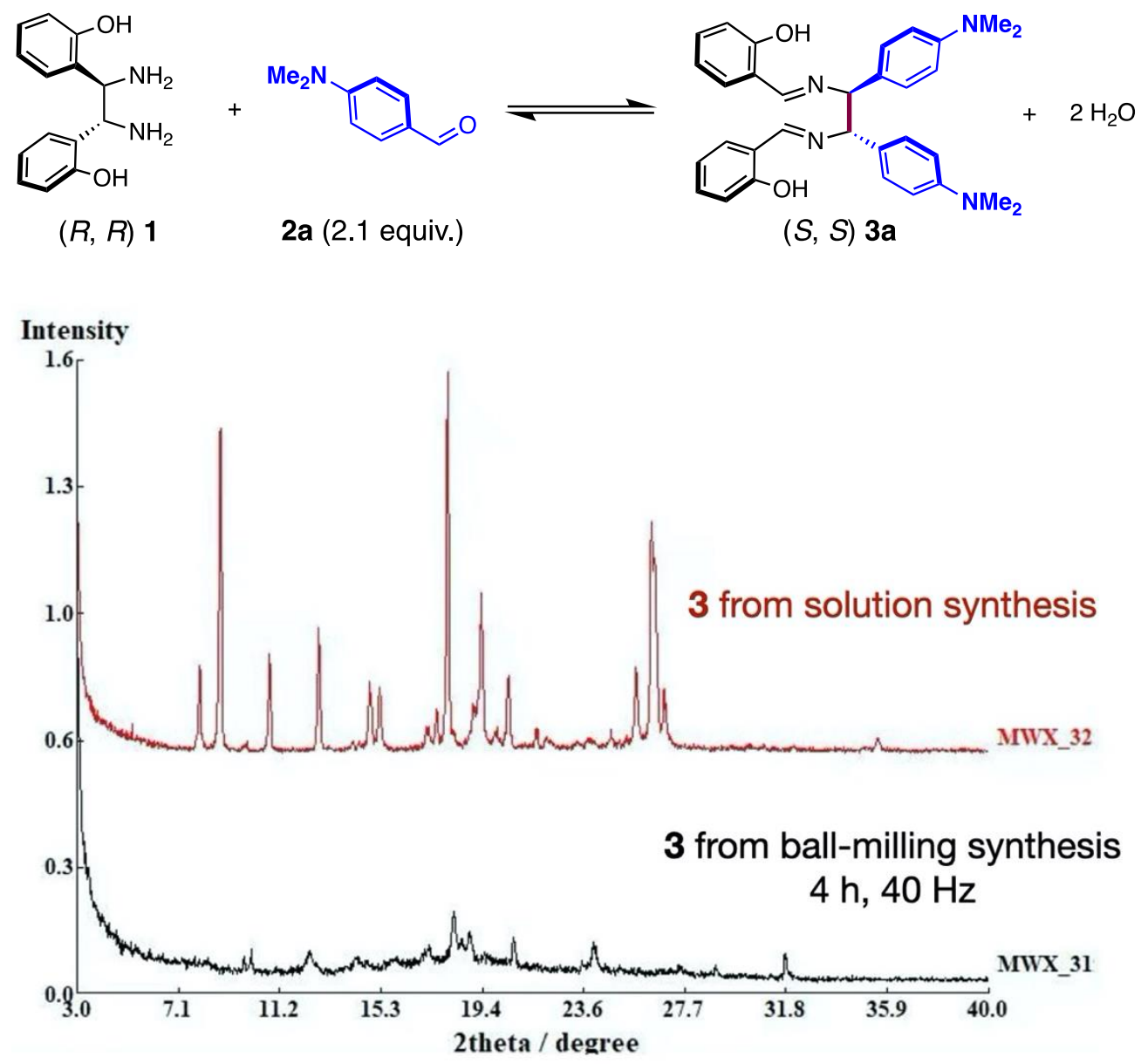

Figure S77a. PXRD patterns of (top) 3a derived from diaza-Cope rearrangement under solutionbased approach, followed by column chromatography purification and solvent removal; (bottom) 3a derived directly from ball-milling reaction after $4 \mathrm{~h}$ at $40 \mathrm{~Hz}$ without any purification. 


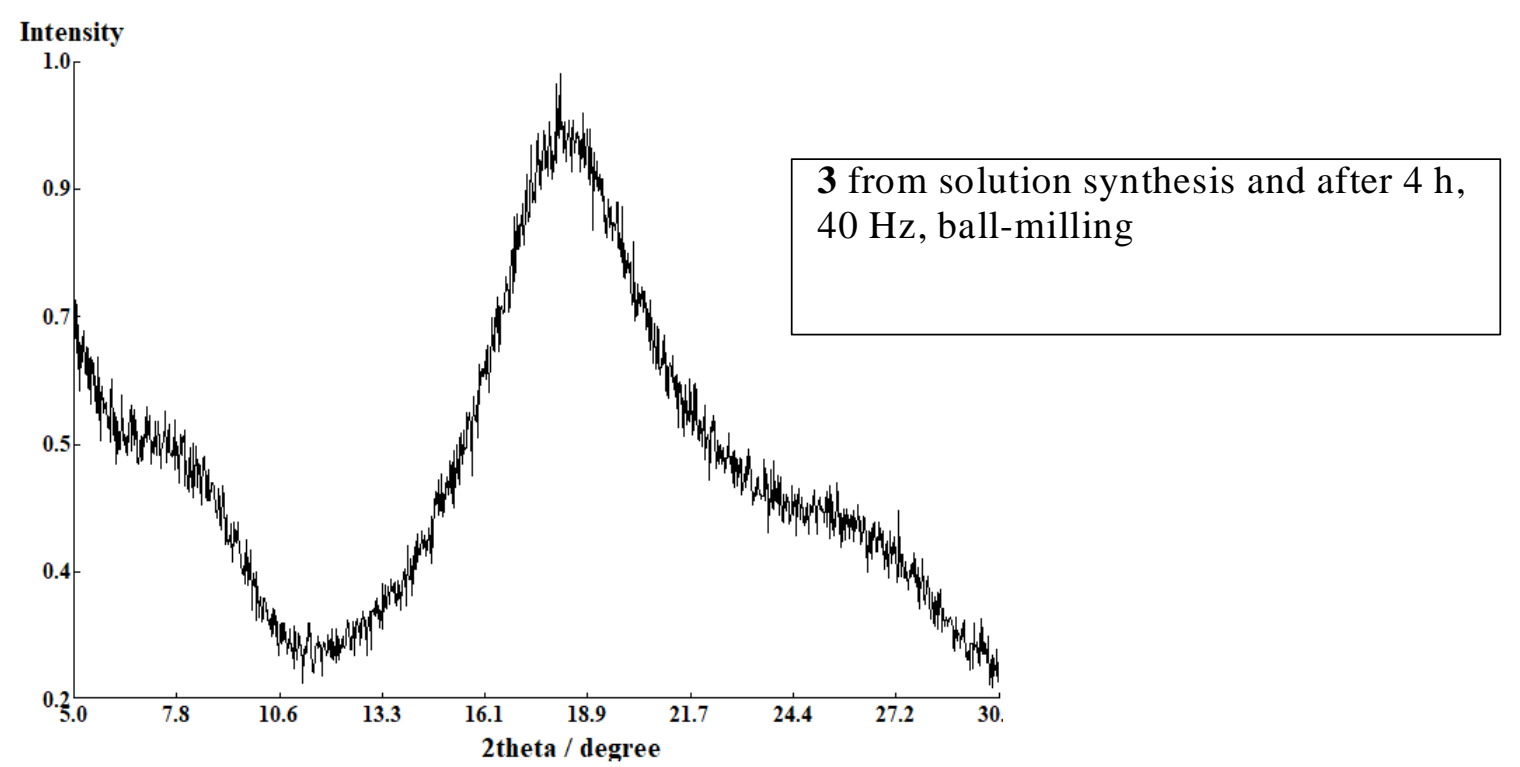

Figure S77b. PXRD patterns of (top) 3a derived from diaza-Cope rearrangement under solutionbased approach, followed by column chromatography purification and solvent removal, followed by ball-milling at $40 \mathrm{~Hz}$ for $4 \mathrm{~h}$.

Comment: 3a subjected from ball-milling reaction clearly generates a crystalline phase (not starting materials) that has a different crystalline phase than one derived from solution-based reaction. When a sample of $\mathbf{3}$, prepared from solution-based reaction, was subject to PXRD experiment, well-defined PXRD peaks were observed (Fig. S77a-top). However, when this powdered sample was subjected to ball-milling at $40 \mathrm{~Hz}$ for $4 \mathrm{~h}$, a feature-less PXRD spectrum was obtained. This suggests that the crystalline phase of $\mathbf{3}$ is destroyed under ball-milling condition (Fig. S77b). 


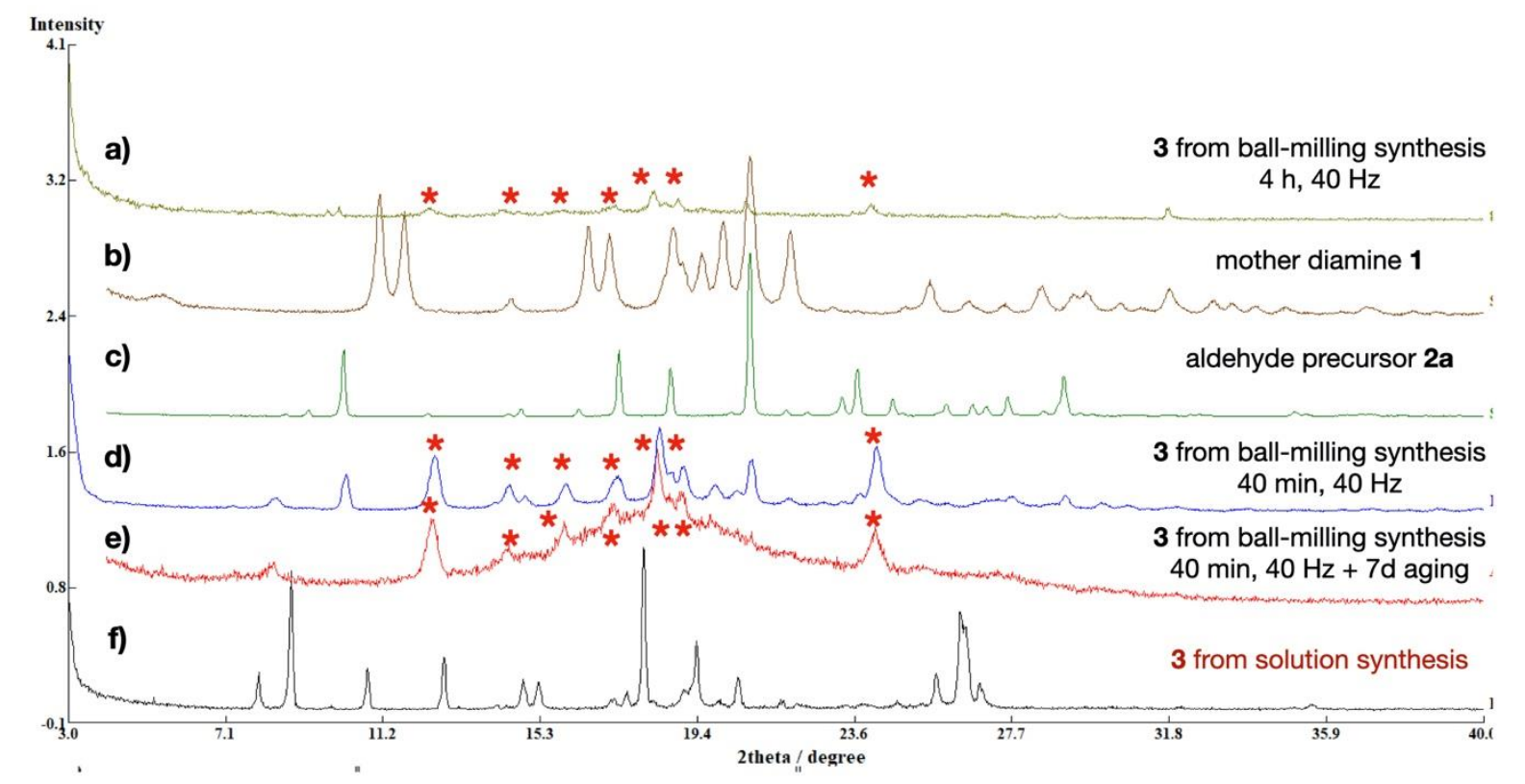

* represents new crystalline phase corresponding to product $3 a$ under mechanochemical synthesis

Figure S78. PXRD patterns of (a) 3a derived directly from ball-milling reaction after $4 \mathrm{~h}$ at 40 Hz without any purification; (b) mother diamine 1; (c) aldehyde precursor 2a; (d) 3a derived directly from ball-milling reaction after $40 \mathrm{~min}$ at $40 \mathrm{~Hz}$ without any purification; (e) 3a derived directly from ball-milling reaction after $40 \mathrm{~min}$ at $40 \mathrm{~Hz}$ without any purification, followed by aging for $7 \mathrm{~d}$ at rt; (f) 3a derived from diaza-Cope rearrangement under solution-based approach, followed by column chromatography purification and solvent removal.

Comment: The new crystalline phase derived from solid-state ball-milling gradually appears from $40 \mathrm{~min}$ to $4 \mathrm{~h}$ of ball-milling. When ball-milling was stopped at $40 \mathrm{~min}$, followed by $\mathrm{rt}$ aging, the intensity of this new phase, which we assigned to formation of $\mathbf{3}$, increases.

All attempts to isolate imidazoline 5 failed. However, based on the amount of 5 observed in ${ }^{1} \mathrm{H}$ NMR spectra at different time points during the course of ball-milling reaction with $\mathbf{1}$ and $\mathbf{2 a}$, we tentatively assigned the new PXRD signals observed (red asterisks in Fig S78) to 5. 


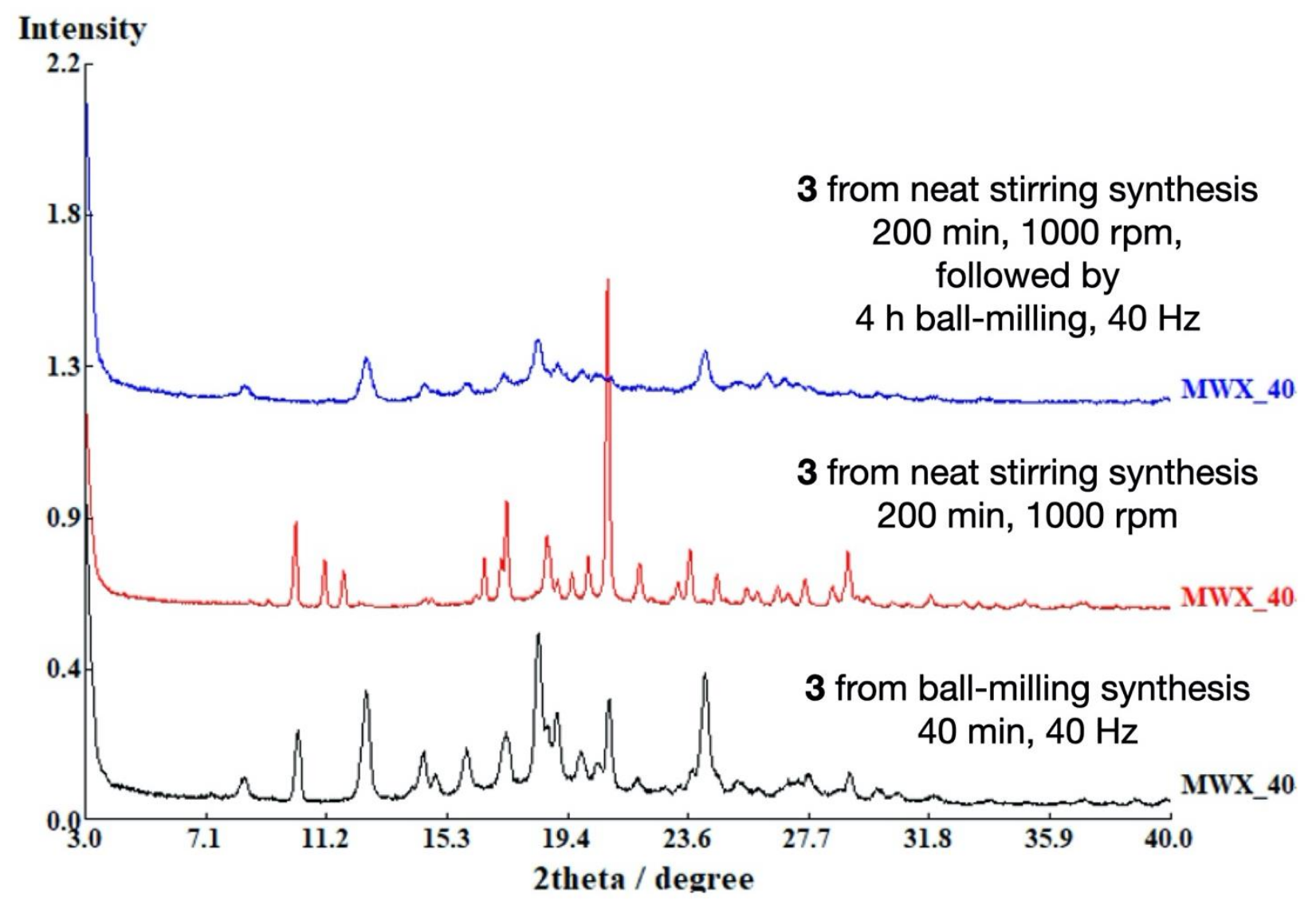

Figure S79. PXRD pattern s of (top) 3a derived from diaza-Cope rearrangement neat stirring approach for $200 \mathrm{~min}$, followed by ball-milling for $4 \mathrm{~h}$ at $40 \mathrm{~Hz}$; (middle) 3a from neat stirring condition for $200 \mathrm{~min}$; and (bottom) 3a derived directly from ball-milling reaction after $40 \mathrm{~min}$ at $40 \mathrm{~Hz}$ without any purification.

Comment: Reaction subjected from neat stirring (physical mixing) recovers mostly starting material $\mathbf{1}$ and 3a, while this mixture upon ball-milling would generate the same crystalline phase of 3a (from ball-milling method). 


\section{HPLC of aging experiments of diaza-Cope rearrangement of $\mathbf{1}$ and $\mathbf{2 a}$}

After mechanochemical reaction between $\mathbf{1}$ and $\mathbf{2 a}$ for $40 \mathrm{~min}$, the sample was kept in centrifuge vial for aging at rt. Periodic HPLC with chiral stationary phase was taken at certain time:

HPLC analysis condition:

Solvent: $n$-hexane/ iso-propanol $=95: 5$

Column: CHIRALPAK IC column (Nomura Chemical Co., Lid.; inner diameter $4.6 \mathrm{~mm} \times$ length 250mm; particle size $=5 \mu \mathrm{m}$ )

Column temperature: $25^{\circ} \mathrm{C}$

Detector: UV at $350 \mathrm{~nm}$

Flow rate: $1.0 \mathrm{~mL} / \mathrm{min}$

Injection volume: $5 \mu \mathrm{L}$

Immediately after ball-milling experiment for $40 \mathrm{~min}$ :

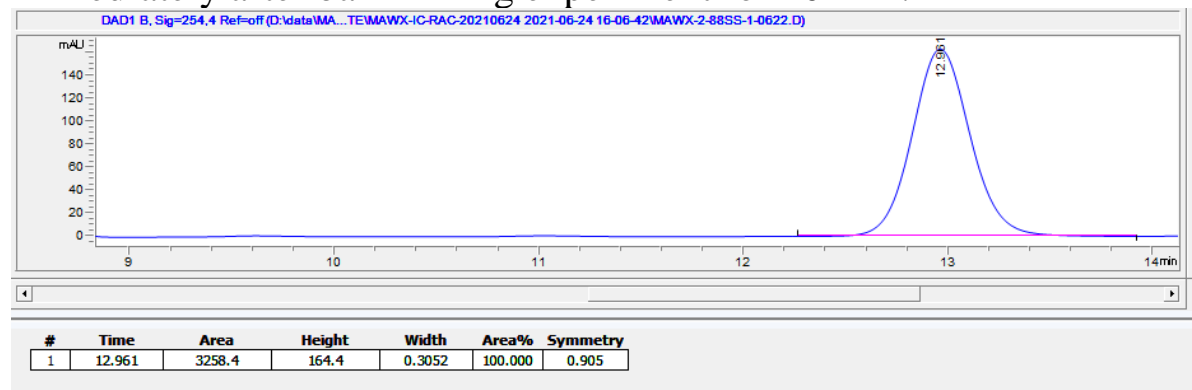

After $10 \mathrm{~h}$ of aging after ball-milling experiment for $40 \mathrm{~min}$ :

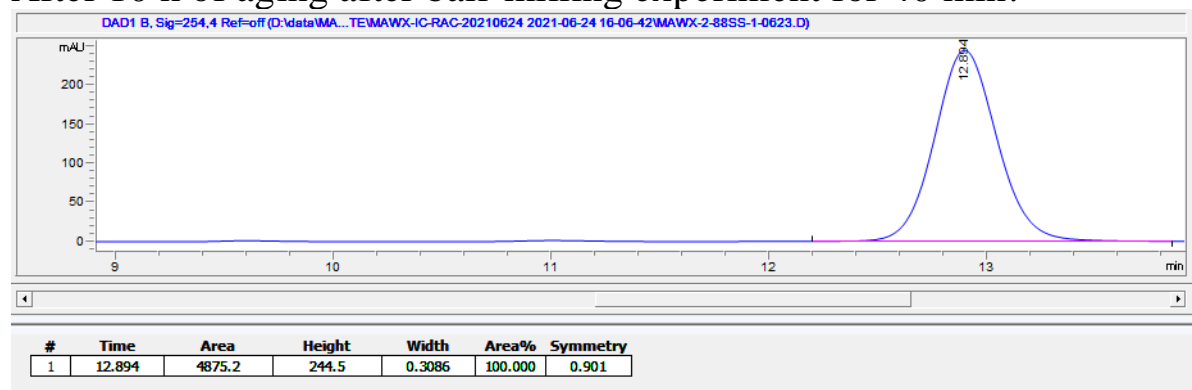

After $3 \mathrm{~d}$ of aging after ball-milling experiment for $40 \mathrm{~min}$ :

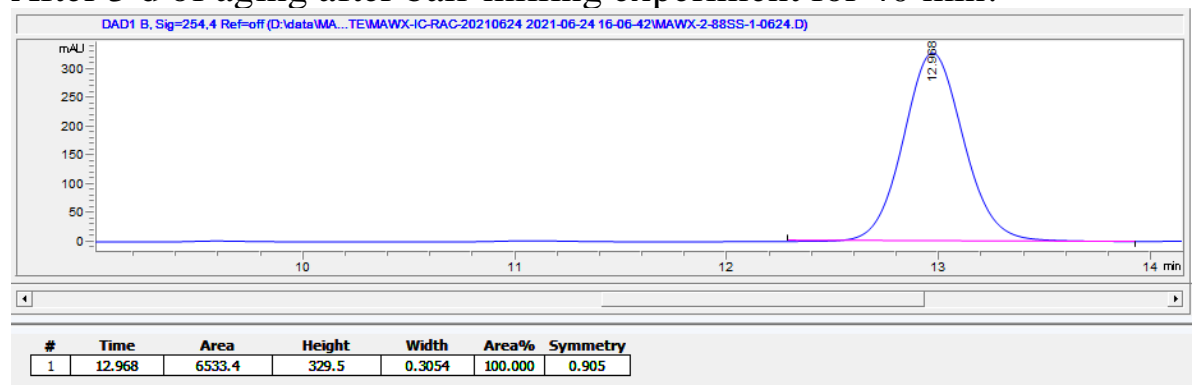

Figure S80. HPLC analysis of (top) immediately after 40 min of ball-milling, (middle) aging for $10 \mathrm{~h}$, and (bottom) aging for $3 \mathrm{~d}$. 
10. Single-crystal X-ray diffraction analysis of $\mathbf{3 1}$

\begin{tabular}{ll}
\hline Identification code & mwx_132_2_0m \\
CCDC number & 2090268 \\
Empirical formula & $\mathrm{C}_{24} \mathrm{H}_{20} \mathrm{~N}_{2} \mathrm{O}_{2} \mathrm{~S}_{2}$ \\
Formula weight & 432.30 \\
Temperature & $150.0 \mathrm{~K}$ \\
Crystal system & Orthorhombic \\
Space group & $P 2_{1} 2_{1} 2_{1}$ \\
& $\mathrm{a}=10.4190(4) \AA$ \\
& $\mathrm{b}=10.9997(4) \AA$ \\
& $\mathrm{c}=19.0495(7) \AA$ \\
Unit cell dimensions & $\alpha=90^{\circ}$ \\
& $\beta=90^{\circ}$ \\
& $\gamma=90^{\circ}$ \\
Volume & $2183.18(14) \AA^{3}$ \\
Z & 4 \\
Density (calculated) & $1.315 \mathrm{~g} / \mathrm{cm}^{3}$ \\
Absorption coefficient & $1.564 \mathrm{~mm} \mathrm{~m}^{-1}$ \\
F(000) & 904.0 \\
Crystal size & $0.2 \times 0.15 \times 0.1 \mathrm{~mm}^{3}$ \\
Radiation & $\mathrm{GaK} \alpha(\lambda=1.34138)$ \\
Theta range for data collection & 8.074 to $114.142^{\circ}$ \\
Index ranges & $-13 \leq \mathrm{h} \leq 12,-13 \leq \mathrm{k} \leq 13,-23 \leq 1 \leq 23$ \\
Reflections collected & 23867 \\
Independent reflections & $4447\left[\mathrm{R}_{\text {int }}=0.0530\right]$ \\
Max. and min. transmission & 0.751 and 0.632 \\
Refinement method & Full-matrix least-squares on $\mathrm{F}^{2}$ \\
Data/restraints/parameters & $4447 / 10 / 266$ \\
Goodness-of-fit on $\mathrm{F}^{2}$ & 1.034 \\
Final R indexes [I $=2 \sigma(\mathrm{I})]$ & $\mathrm{R}_{1}=0.0475, \mathrm{wR} \mathrm{R}_{2}=0.1169$ \\
$\mathrm{R}$ indexes [all data] & $\mathrm{R}_{1}=0.0537, \mathrm{wR} 2=0.1209$ \\
Largest diff. peak and hole & 0.90 and $-0.31 \mathrm{e} \cdot \AA^{-3}$ \\
Flack x parameter & $0.041(37)$ \\
Flack parameter (Parsons' method) & $0.048(8)$ \\
\hline &
\end{tabular}


The ORTEP diagram of the asymmetric unit of $\mathbf{3 1}$ is shown in Figure S80. In this crystal structure, sulfur atom on one of the thiophene ring is disordered in two locations and has partial occupancies with a carbon atom. Using PART command, one location has partial Occ of $28 \%$ and $72 \%$ (PART 2/PART 1; PART $1+$ PART $2=100 \%$ occupancy), and another location has Occ of $29 \%$ and $71 \%$ (PART 3/PART 4; PART 3 + PART $4=100 \%$ occupancy). In our refinement strategy, we applied suitable crystallographic restraints like SADI, FLAT, EADP to model diffused electron densities obtained from difference Fourier map. Absolute stereochemistry of $\mathbf{3 l}$ is unambiguously assigned as $R, R$ configuration with Flack parameter of 0.048(8) using Parsons' method.

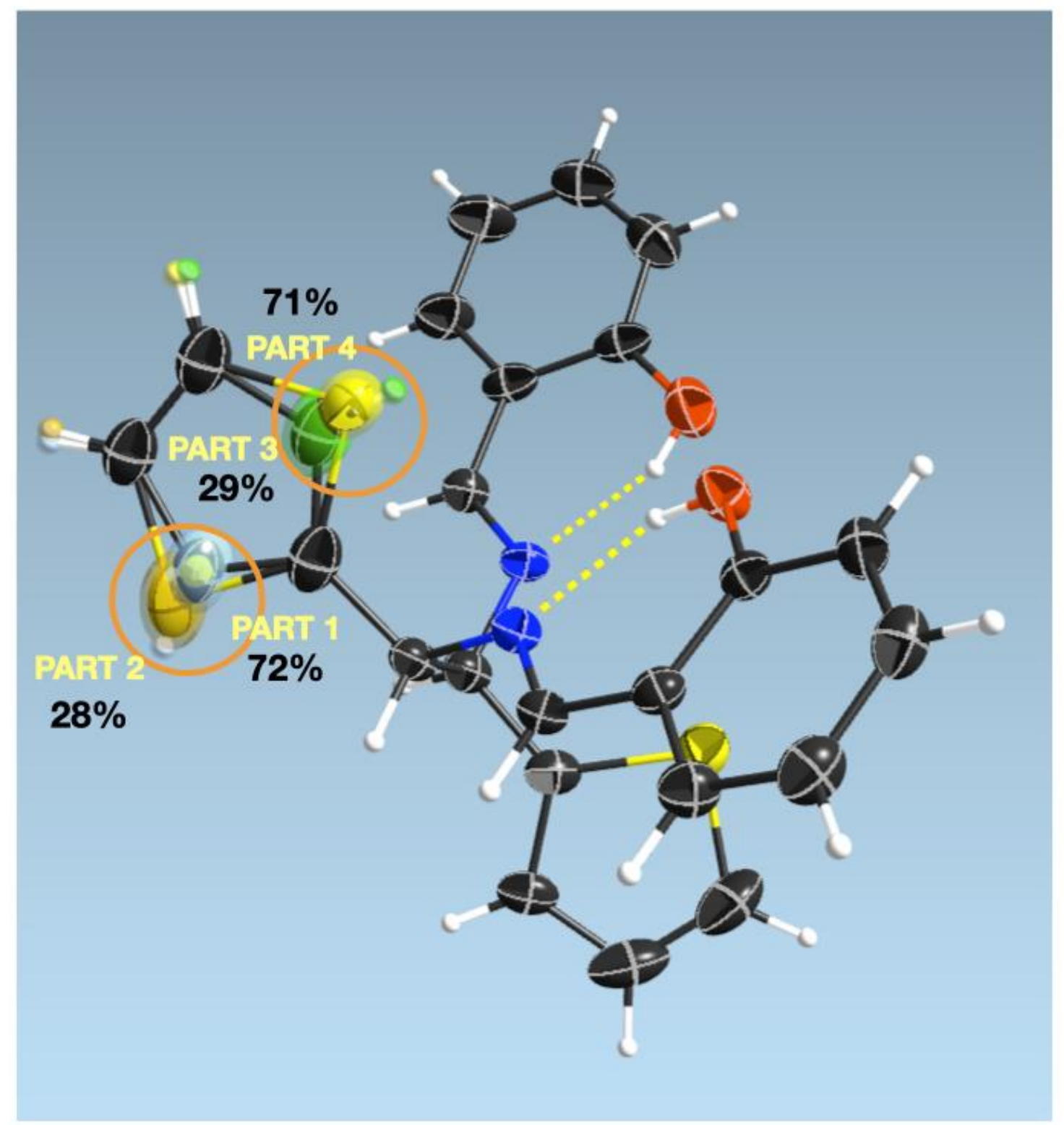

Figure S81. ORTEP diagram of 3I (thermal ellipsoids are shown in 50\% probability). 


\section{Temperature monitoring during mechanochemical conditions}

It is important to keep track on temperature change during ball-milling reactions, in different reaction conditions (ball/jar materials, \# of balls, time). We have monitored the reaction conditions the best as we can using an infrared thermographic camera to check the temperature on the hottest spot on both jar exterior and jar interior. The following tables summarized our finding:

1. We investigated the effect of temperature change by varying \# of balls vs. time (Table S7).

Table S7: Temperature monitor by changing different ball-milling conditions Variable change: \# of ball vs. time

\begin{tabular}{|c|c|c|c|c|c|c|}
\hline Entry & $\begin{array}{c}\text { jar } \\
\text { material/volume }\end{array}$ & $\begin{array}{c}\text { ball } \\
\text { material/size/\# }\end{array}$ & $\begin{array}{c}\text { frequency } \\
(\mathrm{Hz})\end{array}$ & $\begin{array}{c}\text { Time } \\
\text { (h) }\end{array}$ & $\begin{array}{l}\text { jar exterior } \\
\text { temp }\left({ }^{\circ} \mathrm{C}\right)\end{array}$ & $\begin{array}{l}\text { jar interior } \\
\text { temp }\left({ }^{\circ} \mathrm{C}\right)\end{array}$ \\
\hline 1 & $\mathrm{SS} 15 \mathrm{~mL}$ & $\mathrm{SS} / 12 \mathrm{~mm} \times 1$ & 40 & 1 & 36.1 & 53.5 \\
\hline 2 & $\mathrm{SS} 15 \mathrm{~mL}$ & $\mathrm{SS} / 12 \mathrm{~mm} \times 1$ & 40 & 2 & 39.0 & 54.9 \\
\hline 3 & $\mathrm{SS} 15 \mathrm{~mL}$ & $\mathrm{SS} / 12 \mathrm{~mm} \times 1$ & 40 & 3 & 40.5 & 56.0 \\
\hline 4 & $\mathrm{SS} 15 \mathrm{~mL}$ & $\mathrm{SS} / 12 \mathrm{~mm} \times 1$ & 40 & 4 & 41.4 & 56.1 \\
\hline 5 & $\mathrm{SS} 15 \mathrm{~mL}$ & $\mathrm{SS} / 12 \mathrm{~mm} \times 2$ & 40 & 1 & 36.1 & 55.3 \\
\hline 6 & $\mathrm{SS} 15 \mathrm{~mL}$ & $\mathrm{SS} / 12 \mathrm{~mm} \times 2$ & 40 & 2 & 39.2 & 57.5 \\
\hline 7 & $\mathrm{SS} 15 \mathrm{~mL}$ & $\mathrm{SS} / 12 \mathrm{~mm} \times 2$ & 40 & 3 & 41.0 & 56.2 \\
\hline 8 & $\mathrm{SS} 15 \mathrm{~mL}$ & $\mathrm{SS} / 12 \mathrm{~mm} \times 2$ & 40 & 4 & 41.0 & 56.9 \\
\hline 9 & $\mathrm{SS} 15 \mathrm{~mL}$ & $\mathrm{SS} / 12 \mathrm{~mm} \times 3$ & 40 & 1 & 36.2 & 60.6 \\
\hline 10 & $\mathrm{SS} 15 \mathrm{~mL}$ & $\mathrm{SS} / 12 \mathrm{~mm} \times 3$ & 40 & 2 & 39.1 & 62.7 \\
\hline 11 & $\mathrm{SS} 15 \mathrm{~mL}$ & $\mathrm{SS} / 12 \mathrm{~mm} \times 3$ & 40 & 3 & 40.8 & 63.0 \\
\hline 12 & $\mathrm{SS} 15 \mathrm{~mL}$ & $\mathrm{SS} / 12 \mathrm{~mm} \times 3$ & 40 & 4 & 41.2 & 62.2 \\
\hline 13 & $\mathrm{SS} 15 \mathrm{~mL}$ & $\mathrm{SS} / 12 \mathrm{~mm} \times 4$ & 40 & 1 & 36.1 & 54.8 \\
\hline 14 & $\mathrm{SS} 15 \mathrm{~mL}$ & $\mathrm{SS} / 12 \mathrm{~mm} \times 4$ & 40 & 2 & 39.5 & 60.3 \\
\hline 15 & $\mathrm{SS} 15 \mathrm{~mL}$ & $\mathrm{SS} / 12 \mathrm{~mm} \times 4$ & 40 & 3 & 40.7 & 60.0 \\
\hline 16 & $\mathrm{SS} 15 \mathrm{~mL}$ & $\mathrm{SS} / 12 \mathrm{~mm} \times 4$ & 40 & 4 & 40.8 & 60.1 \\
\hline
\end{tabular}

- Temperature spikes in the first hour during ball-milling reaction. After $1 \mathrm{~h}$, the interior temperature becomes constant. The temperature increases the most for entry 9-12 with three SS balls $(12 \mathrm{~mm})$.

- For ball-milling with four SS balls, we think the jar filling level is over certain threshold and it affects the balls' impact at their highest acceleration. 
2. We investigated the effect of temperature change by varying ball size vs. time (Table S8).

Table S8: Temperature monitor by changing different ball-milling conditions Variable change: ball size vs. time

\begin{tabular}{|c|c|c|c|c|c|c|}
\hline Entry & $\begin{array}{c}\text { jar } \\
\text { material/volume }\end{array}$ & $\begin{array}{c}\text { ball } \\
\text { material/size/\# }\end{array}$ & $\begin{array}{c}\text { frequency } \\
(\mathrm{Hz})\end{array}$ & $\begin{array}{l}\text { Time } \\
(\mathrm{h})\end{array}$ & $\begin{array}{l}\text { jar exterior } \\
\text { temp }\left({ }^{\circ} \mathrm{C}\right)\end{array}$ & $\begin{array}{l}\text { jar interior } \\
\text { temp }\left({ }^{\circ} \mathrm{C}\right)\end{array}$ \\
\hline 1 & $\mathrm{SS} 15 \mathrm{~mL}$ & $\mathrm{SS} / 5.2 \mathrm{~mm} \times 1$ & 40 & 1 & 28.0 & 33.1 \\
\hline 2 & $\mathrm{SS} 15 \mathrm{~mL}$ & $\mathrm{SS} / 5.2 \mathrm{~mm} \times 1$ & 40 & 2 & 31.1 & 35.6 \\
\hline 3 & $\mathrm{SS} 15 \mathrm{~mL}$ & $\mathrm{SS} / 5.2 \mathrm{~mm} \times 1$ & 40 & 3 & 31.0 & 37.0 \\
\hline 4 & $\mathrm{SS} 15 \mathrm{~mL}$ & $\mathrm{SS} / 8.0 \mathrm{~mm} \times 1$ & 40 & 1 & 32.4 & 39.1 \\
\hline 5 & $\mathrm{SS} 15 \mathrm{~mL}$ & $\mathrm{SS} / 8.0 \mathrm{~mm} \times 1$ & 40 & 2 & 35.7 & 41.0 \\
\hline 6 & $\mathrm{SS} 15 \mathrm{~mL}$ & $\mathrm{SS} / 8.0 \mathrm{~mm} \times 1$ & 40 & 3 & 37.6 & 41.4 \\
\hline 7 & $\mathrm{SS} 15 \mathrm{~mL}$ & $\mathrm{SS} / 10.0 \mathrm{~mm} \times 1$ & 40 & 1 & 35.0 & 50.5 \\
\hline 8 & $\mathrm{SS} 15 \mathrm{~mL}$ & $\mathrm{SS} / 10.0 \mathrm{~mm} \times 1$ & 40 & 2 & 39.4 & 51.0 \\
\hline 9 & $\mathrm{SS} 15 \mathrm{~mL}$ & $\mathrm{SS} / 10.0 \mathrm{~mm} \times 1$ & 40 & 3 & 39.3 & 51.4 \\
\hline 10 & $\mathrm{SS} 15 \mathrm{~mL}$ & $\mathrm{SS} / 12.0 \mathrm{~mm} \times 1$ & 40 & 1 & 36.0 & 53.5 \\
\hline 11 & $\mathrm{SS} 15 \mathrm{~mL}$ & $\mathrm{SS} / 12.0 \mathrm{~mm} \times 1$ & 40 & 2 & 39.0 & 54.9 \\
\hline 12 & $\mathrm{SS} 15 \mathrm{~mL}$ & $\mathrm{SS} / 12.0 \mathrm{~mm} \times 1$ & 40 & 3 & 40.5 & 56.0 \\
\hline
\end{tabular}

- The results $(5.2 \mathrm{~mm}, 8.0 \mathrm{~mm}, 10.0 \mathrm{~mm}$ and $12.0 \mathrm{~mm})$ are consistent, in which larger grinding ball generates stronger impact and the temperature is thus higher. 
3. We examined the temperature change on the kinetic run we showed in Scheme 3B and Figure $\mathrm{S} 75$ in a 30 min interval for 4 hours.

Table S9: Temperature monitor during kinetic run Variable change: Time

\begin{tabular}{|c|c|c|c|c|c|c|}
\hline Entry & $\begin{array}{c}\text { jar } \\
\text { material/volume }\end{array}$ & $\begin{array}{c}\text { ball } \\
\text { material/size/\# }\end{array}$ & $\begin{array}{c}\text { frequency } \\
(\mathrm{Hz})\end{array}$ & $\begin{array}{c}\text { Time } \\
(\mathrm{h})\end{array}$ & $\begin{array}{c}\text { jar exterior } \\
\text { temp }\left({ }^{\circ} \mathrm{C}\right)\end{array}$ & $\begin{array}{c}\text { jar interior } \\
\text { temp }\left({ }^{\circ} \mathrm{C}\right)\end{array}$ \\
\hline 1 & $\mathrm{SS} 15 \mathrm{~mL}$ & $\mathrm{SS} / 12 \mathrm{~mm} \times 1$ & 40 & 0.5 & 29.9 & 40.4 \\
\hline 2 & $\mathrm{SS} 15 \mathrm{~mL}$ & $\mathrm{SS} / 12 \mathrm{~mm} \times 1$ & 40 & 1.0 & 36.0 & 53.5 \\
\hline 3 & $\mathrm{SS} 15 \mathrm{~mL}$ & $\mathrm{SS} / 12 \mathrm{~mm} \times 1$ & 40 & 1.5 & 38.0 & 53.4 \\
\hline 4 & $\mathrm{SS} 15 \mathrm{~mL}$ & $\mathrm{SS} / 12 \mathrm{~mm} \times 1$ & 40 & 2.0 & 39.0 & 54.9 \\
\hline 5 & $\mathrm{SS} 15 \mathrm{~mL}$ & $\mathrm{SS} / 12 \mathrm{~mm} \times 1$ & 40 & 2.5 & 38.7 & 55.4 \\
\hline 6 & $\mathrm{SS} 15 \mathrm{~mL}$ & $\mathrm{SS} / 12 \mathrm{~mm} \times 1$ & 40 & 3.0 & 40.5 & 56.0 \\
\hline 7 & $\mathrm{SS} 15 \mathrm{~mL}$ & $\mathrm{SS} / 12 \mathrm{~mm} \times 1$ & 40 & 3.5 & 40.1 & 55.9 \\
\hline 8 & $\mathrm{SS} 15 \mathrm{~mL}$ & $\mathrm{SS} / 12 \mathrm{~mm} \times 1$ & 40 & 4.0 & 41.4 & 56.1 \\
\hline
\end{tabular}

- Temperature spikes in the first hour during ball-milling reaction. After $1 \mathrm{~h}$, the interior temperature becomes constant at around $55^{\circ} \mathrm{C}$. 


\section{Neat stirring reaction under thermal condition}

Furthermore, to demonstrate that ball-milling is essential to the rate acceleration phenomenon we observed in diazaCope rearrangement, we attempted another neat stirring condition. Based on the temperature monitoring study in Table S9, the highest temperature it can achieve internally in the jar is $56^{\circ} \mathrm{C}$. At $60^{\circ} \mathrm{C}$, reaction under neat stirring condition at $1000 \mathrm{rpm}$ requires $14 \mathrm{~h}$ to reach $37 \%$ yield, monitored by ${ }^{1} \mathrm{H}$ NMR yield with internal standard, while reaction under ballmilling condition reaches $75 \%$ and even $96 \%$ after $3.3 \mathrm{~h}$ and $4 \mathrm{~h}$, respectively.

Table S10: Reactions under different neat stirring conditions.

\begin{tabular}{|c|c|c|c|c|}
\hline entry & $\begin{array}{c}\text { stir frequency } \\
(\mathrm{rpm})\end{array}$ & Temp $\left({ }^{\circ} \mathrm{C}\right)$ & Time (h) & $\begin{array}{c}\text { NMR yield } \\
(\%)\end{array}$ \\
\hline 1 & 1000 & 25 & 3.3 & 7 \\
\hline 2 & 1000 & 60 & 14 & 37 \\
\hline
\end{tabular}


12. Reference

1. Kim, H.; Nguyen, Y.; Yen, C. P.-H.; Chagal, L.; Lough, A. J.; Kim, B. M.; Chin, J. J. Am. Chem. Soc. 2008, 130, 12184-12191.

2. Dolomanov, O. V.; Bourhis, L. J.; Gildea, R. J.; Howard J. A. K.; Puschmann, H. J. Appl. Crystallogr. 2009, 42, 339-341.

3. Sheldrick, G. Acta Cryst. A 2015, 71, 3-8.

4. Sheldrick, G. Acta Cryst. A, 2008, 64, 112-122. 